Evolution of an interfacial crack on the concrete-embankment boundary

L. Glascoe, T. Antoun, Y. Kanarska, I. Lomove, R. Hall, S. Woodson, J. Smith

November 11, 2013 
This document was prepared as an account of work sponsored by an agency of the United States government. Neither the United States government nor Lawrence Livermore National Security, LLC, nor any of their employees makes any warranty, expressed or implied, or assumes any legal liability or responsibility for the accuracy, completeness, or usefulness of any information, apparatus, product, or process disclosed, or represents that its use would not infringe privately owned rights. Reference herein to any specific commercial product, process, or service by trade name, trademark, manufacturer, or otherwise does not necessarily constitute or imply its endorsement, recommendation, or favoring by the United States government or Lawrence Livermore National Security, LLC. The views and opinions of authors expressed herein do not necessarily state or reflect those of the United States government or Lawrence Livermore National Security, LLC, and shall not be used for advertising or product endorsement purposes.

This work performed under the auspices of the U.S. Department of Energy by Lawrence Livermore National Laboratory under Contract DE-AC52-07NA27344. 


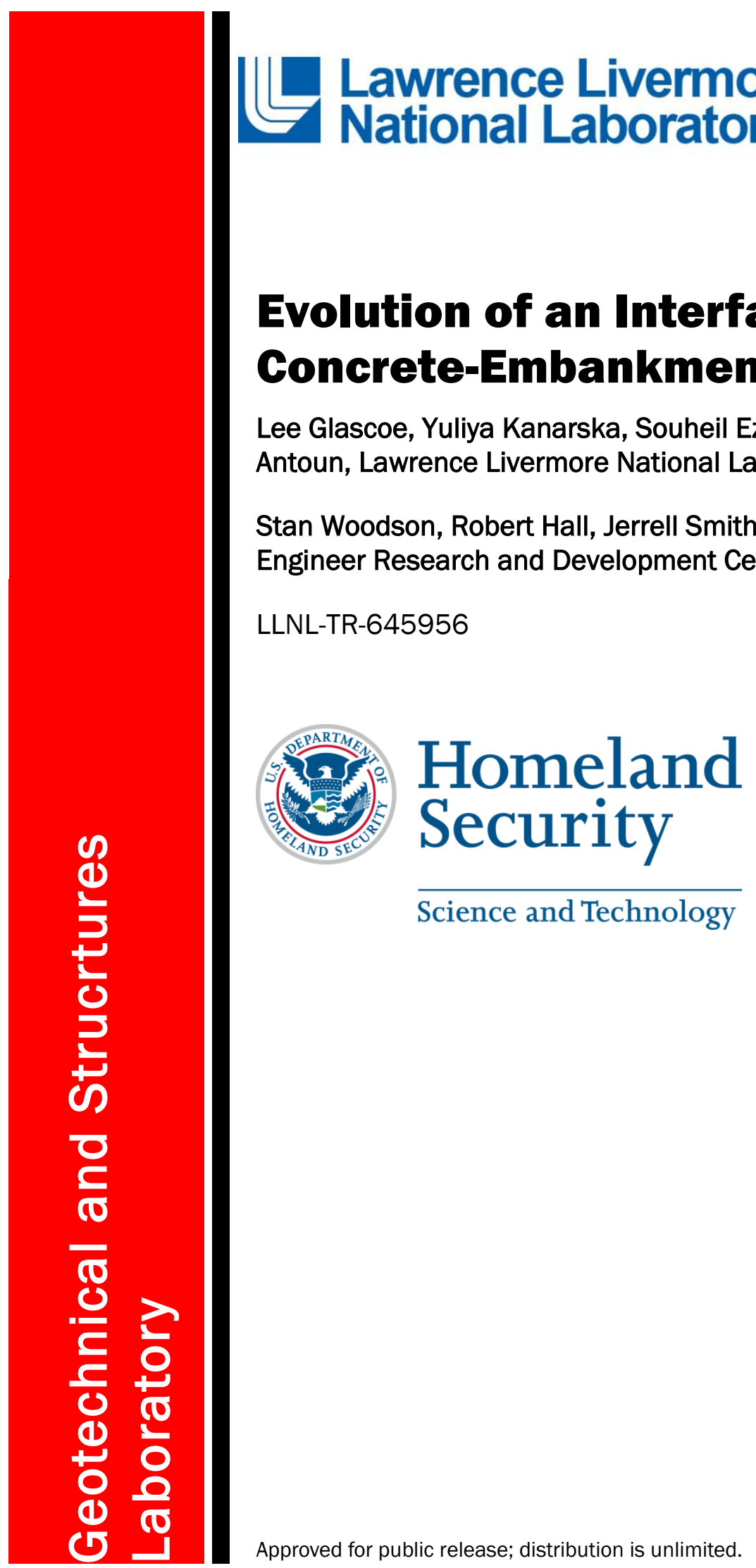


LLNL DISCLAIMER: This document was prepared as an account of work sponsored by an agency of the United States government. Neither the United States government nor Lawrence Livermore National Security, LLC, nor any of their employees makes any warranty, expressed or implied, or assumes any legal liability or responsibility for the accuracy, completeness, or usefulness of any information, apparatus, product, or process disclosed, or represents that its use would not infringe privately owned rights. Reference herein to any specific commercial product, process, or service by trade name, trademark, manufacturer, or otherwise does not necessarily constitute or imply its endorsement, recommendation, or favoring by the United States government or Lawrence Livermore National Security, LLC. The views and opinions of authors expressed herein do not necessarily state or reflect those of the United States government or Lawrence Livermore National Security, LLC, and shall not be used for advertising or product endorsement purposes.

Lawrence Livermore National Laboratory is operated by Lawrence Livermore National Security, LLC, for the U.S. Department of Energy, National Nuclear Security Administration under Contract DE-AC52o7NA27344.

ERDC/USACE: The contents of this report are not to be used for advertising, publication, or promotional purposes. Citation of trade names does not constitute an official endorsement or approval of the use of such commercial products.

The findings of this report are not to be construed as an official Department of the Army position, unless so designated by other authorized documents. 


\section{Evolution of an Interfacial Crack on the Concrete-Embankment Boundary}

Lee Glascoe, Yuliya Kanarska, Souheil Ezzedine, Ilya Lomov, and Tarabay Antoun, Lawrence Livermore National Laboratory

Stan Woodson, Robert Hall, Jerrell Smith, US Army Corps of Engineers Engineer Research and Development Center

Final report

Approved for public release; distribution is unlimited.

Prepared for the Department of Homeland Security Science and Technology Directorate Washington, DC 20314-1000 


\begin{abstract}
:
Failure of a dam can have subtle beginnings. A small crack or dislocation at the interface of the concrete dam and the surrounding embankment soil initiated by, for example, a seismic or an explosive event can lead to a catastrophic failure of the dam. The dam may 'self-rehabilitate' if a properly designed granular filter is engineered around the embankment. Currently, the design criteria for such filters have only been based on experimental studies. We demonstrate the numerical prediction of filter effectiveness at the soil grain scale. This joint LLNL-ERDC basic research project, funded by the Department of Homeland Security's Science and Technology Directorate (DHS S\&T), consists of validating advanced high performance computer simulations of soil erosion and transport of grain- and dam-scale models to detailed centrifuge and soil erosion tests. Validated computer predictions highlight that a resilient filter is consistent with the current design specifications for dam filters. These predictive simulations, unlike the design specifications, can be used to assess filter success or failure under different soil or loading conditions and can lead to meaningful estimates of the timing and nature of full-scale dam failure.
\end{abstract}




\section{Contents}

executive summary

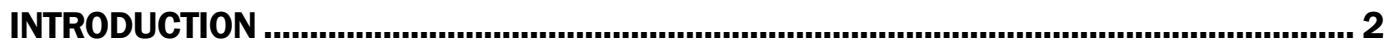

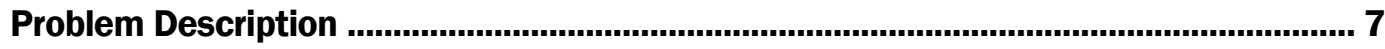

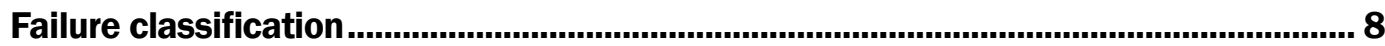

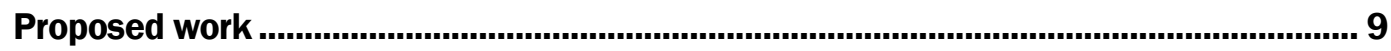

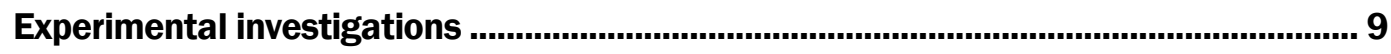

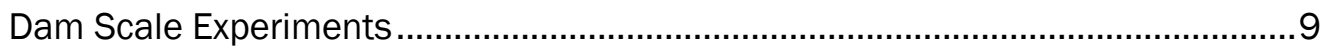

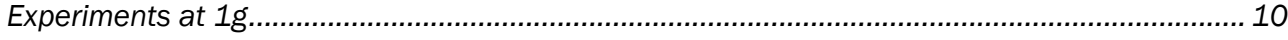

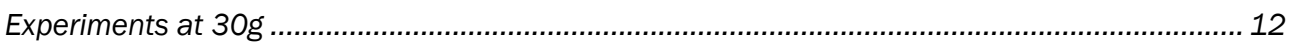

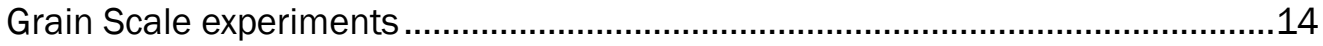

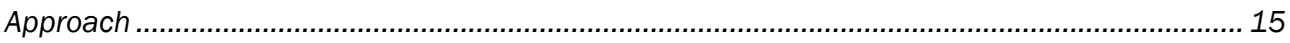

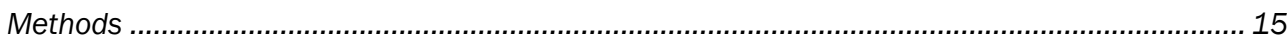

Filter Media and Transportable Particles ........................................................................... 16

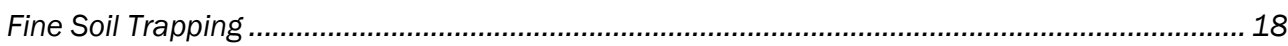

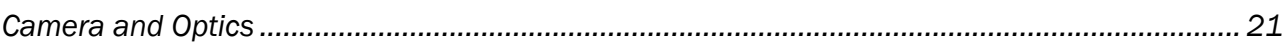

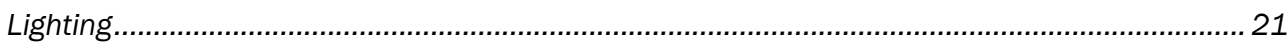

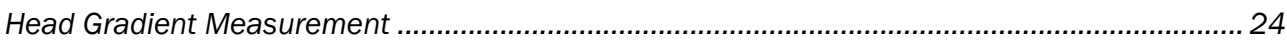

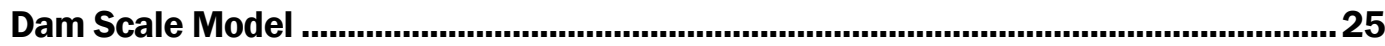

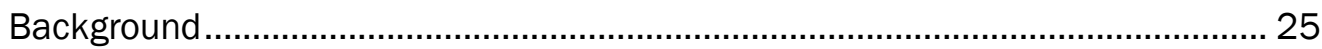

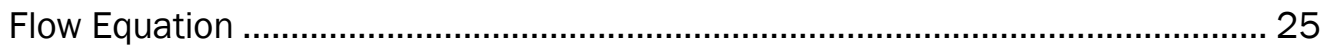

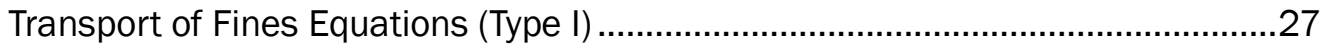

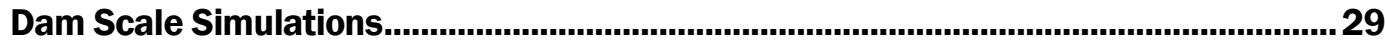

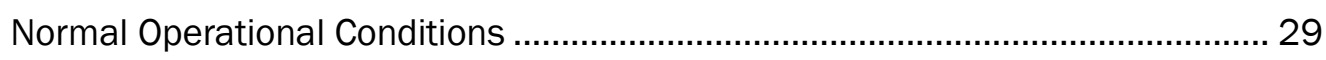

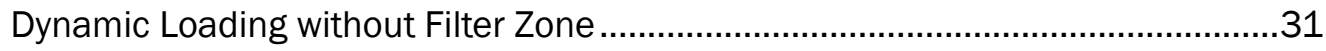

Dynamic Loading with Filter Zone .................................................................. 33

Dual population continuum model for transport of fines (Type II)...................................36

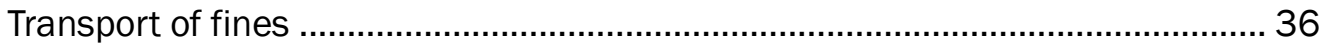

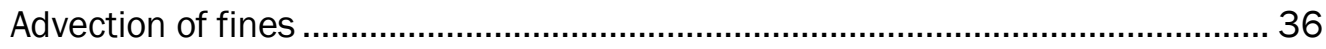

Kinetics and dynamics of fine deposition ......................................................... 41

Simulation of embankment after dynamic loading with attachment Type II ........ 42

Numerical investigation at the grain scale ......................................................................43

Soil erosion in a concrete-wraparound crack ................................................... 43

Behavior of gravel in filter zones ...................................................................4

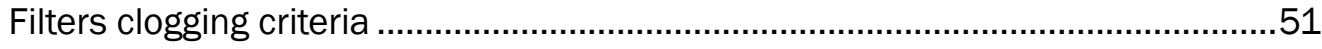

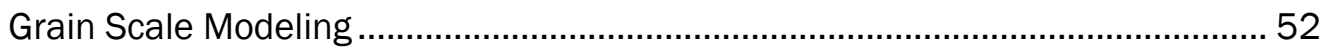


Grain Scale Soil Erosion Simulations ................................................................. 54

Particle and Fluid Transport in Filter Layers ...................................................... 63

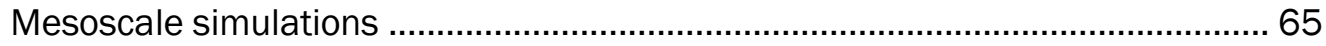

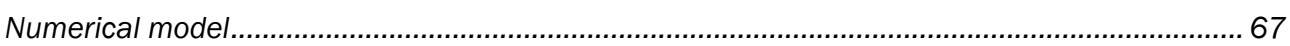

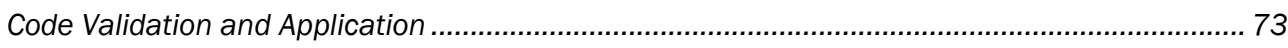

Comparison of SIMULATIONS with Experimental Data ........................................91

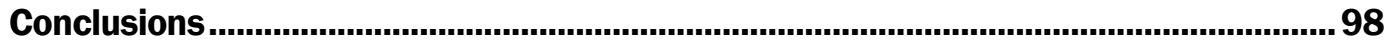

Identified gaps and path forward .........................................................................101

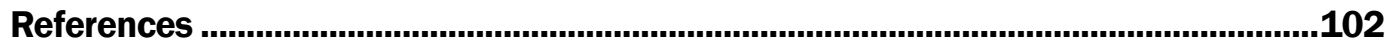

\section{Figures and Tables}

\section{Figures}

Figure 1. Extensional crack. Northwest-trending extensional crack where dam fill settled about $0.6 \mathrm{~m}(2 \mathrm{ft})$ and pulled away from concrete spillway and north abutment of Austrian Dam in the Santa Cruz Mountains, CA, USA. Photograph taken on October 17, 1989 by R.J. McLaughlin, U.S. Geology Survey. 2

Figure 2. Austrian Dam (Santa Cruz, CA). [Top] aerial view, hashed areas represent damaged zones [Bottom] cross-section (Babbit, www.michigan.gov/documents/deq/deq-p2cacaseismicpaper_281049_7.pdf).

Figure 3. Schematic description of typical layers for concrete dam embankment. (a) Aerial view. (b), (c) side-slice views. Layers of embankment, water level, and phreatic line (the line corresponding to the phreatic surface lying above the saturated zone when seen in a vertical plane) are shown 5

Figure 4. Construction of the wrap-around section of Folsom Dam .....................................6

Figure 5. Test set up for embankment with silt core and transition zones. ......................... 10

Figure 6. Indication of seepage at tip of crack at the upper section and at toe. ................ 11

Figure 7. Downstream cracks and drying wet spot at toe, no flow through....................... 11

Figure 8. Seepage through horizontal crack and base of concrete section. ..................... 12

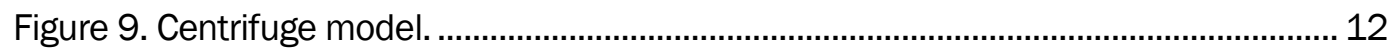

Figure 10. Displacement plot of crest of concrete gravity section................................... 13

Figure 11. Downstream cracks at crest displacement of $2.1 \mathrm{in}$........................................ 14

Figure 12. Constant head flow chamber, mounted in testing frame. Chamber cross-section is 10 $\mathrm{cm} \times 10 \mathrm{~cm}$ and $20 \mathrm{~cm}$ in length. 15

Figure 13. Filter screen for retaining filter media. Also visible is the flow distribution plate, which provides flow pathways between the inlet and outlet tubing and the filter media. 16

Figure 14. Experimental layout. Pump in supply tank provides flow to head tank and degassing column. Flow from head tank travels through tubing, through flow chamber, and back to supply tank. Head difference between head tank and supply tank drives flow through flow chamber. Hydraulic lift is used to adjust head difference (range 0.2 to $2.0 \mathrm{~m}$ ). 17 
Figure 15. A) Gas bubbles that have precipitated from solution onto glass marbles used in initial testing experiments. B) Bio-barrels which provide a large surface area for degassing water within the degassing column. 20

Figure 16. Transport of a silt particle through $1,500 \mu \mathrm{m}$, spherical filter media. Frame rate is 15 fps. 23

Figure 17. Particle trajectories for silt particles passing through 1,500 $\mu \mathrm{m}$, spherical filter medium. Individual trajectories are indicated by color. . 24

Figure 18. Experimental fluid mean velocity (derived from provided flow rates) and hydraulic gradients in the clean layer. 24

Figure 19. [Not to scale] Schematic of the physical domain for the computational analyses of dam stability under normal operational conditions and after dynamic loading. The blue line represents the water level upstream, through the dam, and downstream of the reservoir. The free surface is also known as phreatic surface. 25

Figure 20. Example of FEM-AMR solution to pressure distribution within an embankment. The embankment is composed of a single core with a very low hydraulic conductivity. Upstream and downstream of the core are relatively high filling. Top: FEM-AMR mesh capturing the free surface. Middle: head (pressure) distribution along the embankment. Bottom: composed solution.26

Figure 21. Example of FEM-AMR around the free surface. At each stage the number of nodes, and thus element, increases around the free surface to capture the solution. The number of nodes are $\sim 2,000, \sim 14,300$, and $\sim 44,750$ for stages 1,2 , and 3 , respectively (from top to bottom). 30

Figure 22. Hydraulic conductivity, $\mathrm{K}$, within the embankment. The distribution of the permeability reflects the 'design' of the embankment. The difference in the hydraulic conductivity between the filling and the core is about 6 orders of magnitude. 30

Figure 23. Time-evolution (snapshots) of fine migration within the embankment (continued). Time flows from left to right and top to bottom. 32

Figure 24. (Continued) Time-evolution (snapshots) of fine migration within the embankment. Time flows from left to right and top to bottom. Simulations shown here do not include attachment of the fine particles; once mobilized they are washed out. 33

Figure 25. Head distribution under normal condition of embankment (top) and under dynamic load (middle). Head distribution and streamlines under dynamic load (bottom). Streamlines show convergence of the flow toward the high conductivity area. High conductivity area is due to dislocation of the embankment from the core of the dam. The creation of high conductivity zone impacts the head distribution and the shape of the free surface. 34

Figure 26. Time-evolution (snapshots) of fine migration within the embankment. Time flows from left to right and top to bottom. Simulations shown here do not include attachment of the fine particles; once mobilized they are washed out. However, the mobilization of fine particles leads to a high conductivity zone: zone of dislocation of embankment from the core of the dam.35

Figure 27. Time-evolution (snapshots) of fine entrapment (filtrations) within the embankment. Time flows from left to right and top to bottom. Simulations shown here do include attachment of the fine particles; once mobilized they are either attached or washed out. Attachment "isotherm" is $F\left(\sigma / \eta_{0}\right)=1-\beta \sigma$. 37

Figure 28. Time-evolution (snapshots) of fine migration (washed) within the embankment. Time flows from left to right and top to bottom. Simulations shown here do include attachment of the fine particles; once mobilized they are either attached or washed out. Attachment "isotherm" is $\mathrm{F}\left(\sigma / \eta_{0}\right)=1-\beta \sigma$. 38 
Figure 29. Time-evolution (snapshots) of fine Entrapment (filtration) within the embankment. Time flows from left to right and top to bottom. Simulations shown here do include attachment of the fine particles; once mobilized they are either attached or washed out. Attachment "isotherm" is $F\left(\sigma / \eta_{0}\right)=1-\beta^{2} \sigma^{2}$. 39

Figure 30. Time-evolution (snapshots) of fine migration (washed) within the embankment. Time flows from left to right and top to bottom. Simulations shown here do include attachment of the fine particles; once mobilized they are either attached or washed out. Attachment "isotherm" is $\mathrm{F}\left(\sigma / \eta_{0}\right)=1-\beta^{2} \sigma^{2}$ 40

Figure 31. Front migration as function of depth and different times for Type I and Type II fine migration models.

Figure 32. Numerical simulation of multiphase flow in an interfacial gap between concrete and soil (HET experiment). Top frame: initial conditions, bed not subject to erosion. Bottom frame: bed is subject to horizontal flow. 47

Figure 33. Numerical simulation of JET experiment. 48

Figure 34. Simulation of the fluid migration through the gravel filter. Hydraulic pressure drop (left panel) and fluid velocity (right panel). 49

Figure 35. Simulation of the fluid migration through the gravel filter with local inhomogenities in packing in the vertical plane (a) and horizontal plane (b), difference in hydraulic pressure drop (c). 49

Figure 36. Gravel motion through a crack. (a) Initial setup. (b) Particles and flow field at 0.5 seconds. The magnitude of a velocity field is shown in color. 50

Figure 37. Shields diagram (adapted from Miedema, 2008) shows relationship between dimensionless shear stresses (Shields parameter) and particle Reynolds number. Shields curves proposed by different authors (Soulsby 1997; Miedema 2008) are shown. Please note that Shields curves are derived for non-cohesive soils. 53

Figure 38. Hjulstrom and Shields diagrams converted to mean flow velocity (adopted from Miedema, 2008). Hjulstrom diagram (bold gray line and dark blue line) shows the relationship between stream velocity and particle erosion, transport and deposition. Bold gray line shows erosion velocity derived by Hjulstrom (1935) for cohesive sediments. 53

Figure 39. Simulations of erosion of 400 micron particles (spheres and irregular particles. 56

Figure 40. Examples of stress and averaged velocity profile for spheres of size 800 and 200 microns at the moment of particle detachment from the surface. 57

Figure 41. Shear stress distribution in simulations. Local heterogeneities in geometry of granular bed result in discrepancy between mean shear stress and local shear stress even in laminar regime. 58

Figure 42. The experimentally derived critical shear stress velocities in comparison to correlations between critical shear stress velocity and grain size from literature. The symbols distinguish between the different used sample types sieved sediment fraction and naturally composed sediments (Bohling et al. 2007). The threshold curve for non-cohesive sediments is based on a modified Shields (1936) curve according to Soulsby and Whitehouse (1997). The curve for cohesive sediments is an adjustment to the Hjulstrom (1935) plot given by Zanke (1977). This graphic is adopted from Bohling et al. (2007) paper. Irregular shapes are also plotted. . 58 Figure 43. Comparison of pickup velocities in the experiments of Dasani et al. (2010), and the current numerical simulations. 60 
Figure 44. An example of polynomial fit for granular bed evolution of 100 and 400 micron spheres.

Figure 45. An example of estimation of parameters for erosion law based on numerical simulations for 400 microns particles of spherical and irregular shape.

Figure 46. Code prediction for core particles migration through the filter for $D^{\text {filter }} \backslash d^{\text {soil }}=4$ (a) and $D^{\text {filter }} \backslash d^{\text {soil }}=10$ (b). Changes in permeability of the filter with time (c). 64

Figure 47. Soil migration through "damaged" filter. (a) case of effective filter with moderate damage. Soil particles effectively clog increased pore spaces. (b) case of non-effective filter with significant damage. Soil particles are washed out through the crack and are not retained by the filter. The color scale for velocity field is from 0 to $0.05 \mathrm{~m} / \mathrm{s}$. 64

Figure 48. Comparison of soil and fluid fluxes in moderately damaged filter and significantly damaged filter. 65

Figure 49. Computational domain and geometry of AMR patches for simulation of fluid flow through the cubic array of spheres. 74

Figure 50. Comparison of the computed friction coefficient with available data for random and cubic packing $s$ at $\operatorname{Re}=18$ (left panel) and $R e=900$ (right panel). Data from Hovekamp (2002), Vortwek and Brunn (1994), Ergun (1952), Franzen (1979), and Martin et al. (1951) are shown. 75

Figure 51. Horizontal velocities (in $\mathrm{m} / \mathrm{s}$ ) at the central slice ( $\mathrm{x}-\mathrm{z}$ plane at $\mathrm{y}=0.004 \mathrm{~m}$ ) for the cubic packing of spheres at $\mathrm{Re}=18$. 75

Figure 52. Convergence rate for $\mathrm{Re}=18$. Numerical error versus resolution is shown. .....76 Figure 53. Horizontal velocities (in $\mathrm{m} / \mathrm{s}$ ) at the central slice ( $\mathrm{x}-\mathrm{z}$ plane at $\mathrm{y}=0.004 \mathrm{~m}$ ) for the cubic packing of spheres at $\mathrm{Re}=900$. 77

Figure 54. Convergence rate for $\mathrm{Re}=900$. Numerical error versus resolution is shown... 77 Figure 55. Pressure drop (in $\mathrm{Pa}$ ) through the randomly packed bed.................................... 78 Figure 56. Convergence rate. Numerical error versus resolution is shown....................... 78 Figure 57. Two different packings with the same porosity of $42 \%$. In configuration 1 (a) the anisotropy in the vertical $x$-y plane is introduced by removing few particles from the initial randomly packed structure with porosity $36 \%$ at $z=0.8 \mathrm{~m}$. In configuration 2 (b) the anisotropy in the horizontal $y_{-} z$ plane is introduced in the same manner at $x=0.3 \mathrm{~m}$. 79

Figure 58. Comparison of the pressure drop in configuration 1, configuration 2 and regular random packing with porosity $42 \%$. 79

Figure 59. Averaged packing density for configuration 1 (a) and configuration 2 (b).......80 Figure 60 . Schematic representation of two layers with different porosity and volume to estimate averaged properties of the combined medium. 81

Figure 61. Numerical simulation of falling particle in a Newtonian fluid. A vertical velocity (in $\mathrm{m} / \mathrm{s}$ ) and pressure distribution (in $\mathrm{Pa}$ ) are shown. 82

Figure 62. Comparison of settling velocity for falling particle example (3D) with analytical Stokes solution Stokes (1966) and Heywood tabulated data Heywood (1948) for different Reynolds numbers (left column). Convergence study for different Reynolds numbers (right column).83 Figure 63. AMR grid in simulations of falling particle in a Newtonian fluid. 84 Figure 64. Schematic description of experimental setup performed at the U.C. Berkeley Thermal Hydraulics Lab. 86 
Figure 65. Comparison of the pebble flow in experiment and simulations at time $9.5 \mathrm{~s}$ (left panel) and $28.5 \mathrm{~s}$ (right panel) 87

Figure 66. Comparison between experiment and simulations for the bed position below the chute outlet.

Figure 67. Effect of the damping coefficient on the pebble mass flux in the chute section. The pebble mass flux is shown relative to the pebble mass flux with no damping. Note that the damping coefficient is connected to the restitution coefficient Tsuji et al. (1992). Damping coefficient 0 corresponds to no damping and restitution coefficient 1 , damping coefficient 1 corresponds to fully damped situation with restitution coefficient 0 . 88

Figure 68. Effect of the stiffness coefficient on the pebble mass flux. The axis shows normalized numerical stiffness using theoretical Hertzian value stiffness ST. 90

Figure 69. Schematic description of experimental and numerical setups......................... 91

Figure 70. Simulations of base particle clogging in the filter layer. 93

Figure 71. Comparison between simulations and experiment for fluid mean velocity (derived from provided flow rates) and hydraulic gradients in the clean layer. 94

Figure 72. Comparison between simulated and experimental mass fraction of the base particles in the filter layer. 94

Figure 73. Uniform distribution of the base particles (left) and partial distribution of the base particles (right). 97

Figure 74. Comparison of the fluid mean velocity evolution (left) and hydraulic gradient in clean layer (right) for uniform and partial base particle sources. .97

Figure 75. Schematic description of the pressure drops in simulations and experiment.98

\section{Tables}

Table 1. Materials for filter media and transportable particles in experiments. ................17

Table 2. Proposed experimental combinations. ................................................................... 19

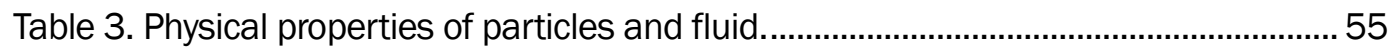

Table 4. Numerical results from simulations with spherical particles................................. 62

Table 5. Numerical results from simulations with irregular shapes..................................... 62

Table 6 Time per CPU in seconds to perform a single computed time step as a function of the number of processors and the resolution for the falling particle. ........................................ 84

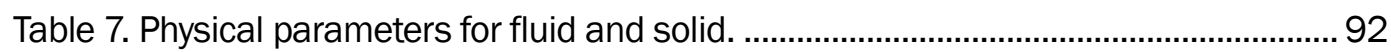

Table 8. Comparisons between experiment and simulations............................................. 96 


\section{Unit Conversion Factors}

\begin{tabular}{|c|c|c|}
\hline Multiply & By & To Obtain \\
\hline British thermal units (International Table) & $1,055.056$ & Joules \\
\hline cubic feet & 0.02831685 & cubic meters \\
\hline cubic inches & $1.638706410^{-5}$ & cubic meters \\
\hline degrees Fahrenheit & $(F-32) / 1.8$ & degrees Celsius \\
\hline Feet & 0.3048 & meters \\
\hline Inches & 0.0254 & meters \\
\hline pounds (mass) per square yard & 0.542492 & kilograms per square meter \\
\hline square feet & 0.09290304 & square meters \\
\hline square inches & $6.451610^{-4}$ & square meters \\
\hline
\end{tabular}




\section{EXECUTIVE SUMMARY}

Failure of a dam can have subtle beginnings. A small crack or dislocation at the interface of the concrete dam and the surrounding embankment soil initiated by, for example, a seismic or an explosive event can lead to a catastrophic failure of the dam. The dam may 'self-rehabilitate' if a properly designed granular filter is engineered around the embankment. Granular filters are one of the most important protective design elements of large, zoned embankment dams. In case of cracking and erosion, if the filter is capable of retaining the eroded particles, then the crack will seal and the dam safety will be ensured. Currently, the design criteria for such filters have only been based on experimental studies. We demonstrate the numerical prediction of filter effectiveness at the soil grain scale. This joint LLNL-ERDC basic research project, funded by the Department of Homeland Security's Science and Technology Directorate (DHS S\&T), consists of validating advanced high performance computer simulations of soil erosion and transport to detailed centrifuge and soil erosion tests. Validated computer predictions highlight that a resilient filter is consistent with the current design specifications for dam filters. These predictive simulations, unlike the design specifications, can be used to assess filter success or failure under different soil or loading conditions and can lead to meaningful estimates of the timing and nature of full-scale dam failure. Simulations and experiments comparison show good qualitative and quantitative agreement at both the grain and the dam scales. For a more precise understanding of the soil transport in granular filters we investigated the sensitivity of the particle clogging mechanism to various aspects such as particle size ration, the amplitude of hydraulic gradient, particle concentration and contact properties. We found that for intermediate particle size ratios the high flow rates and low friction lead to deeper intruding depths. We also found that and the damage tends to be shallower and more severe with decreasing flow rate, increasing friction and concentration of suspended particles. Finally we provide a roadmap for future work to better resolve scaling issues with more complete soil constitutive models and to better account for probabilistic variability of soil and hydraulic properties associated with large scale dam failure. 


\section{INTRODUCTION}

There are many crack-initiating events that can result in the catastrophic failure of embankments dams. Transverse cracks in embankment dams can develop as a result of postconstruction settlement ${ }^{1}$, earthquake deformations ${ }^{2}$, or anthropogenic loads such as emplacedexplosives. Damaging the filter or core materials has the potential to initiate systematic failure of the zoned embankment. For example, Matahina Dam in New Zealand suffered settlement and cracking as results of the magnitude 6.3 Edgecumbe Earthquake on May 2, 1987. Trenching showed that the fissures were shallow and did not extend across the core. The trenching exposed a large cavity, which was thought to be related to an earlier core cracking, seepage, and internal erosion. This example is a testimonial case of the "self-rehabilitation" of the dam under conditions of failure. It was, however, observed that the rate of seepage through the dam has increased as a result of the earthquake.

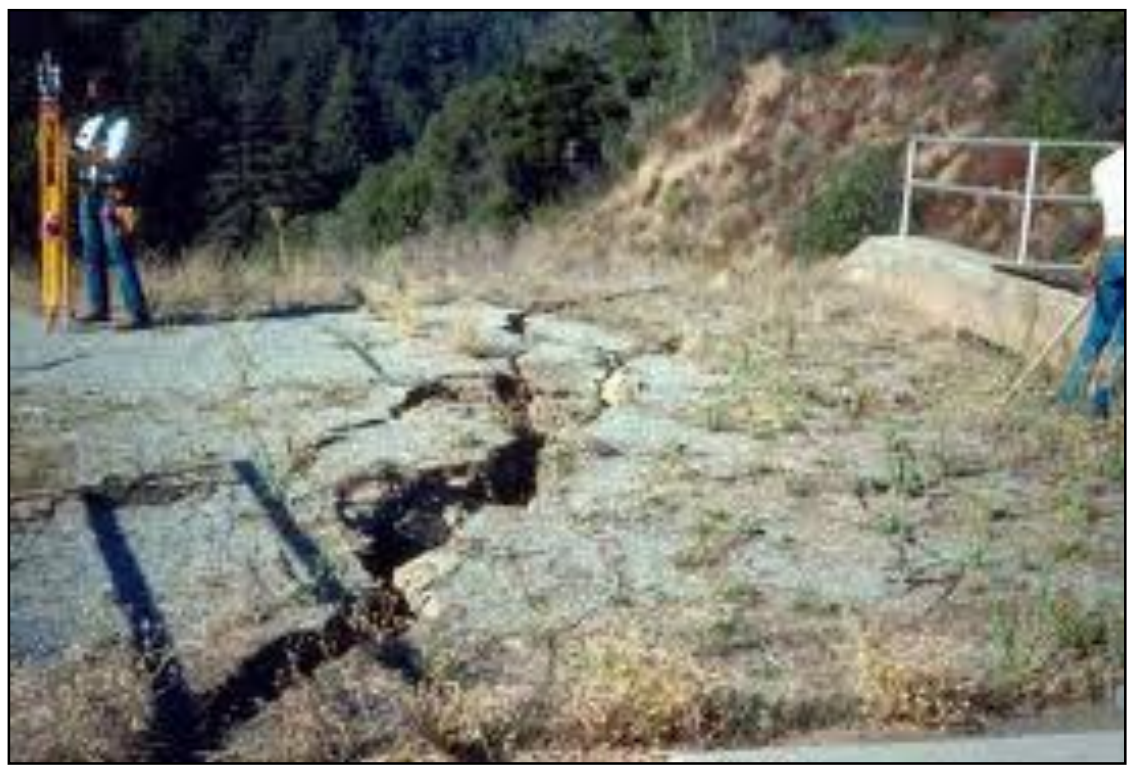

Figure 1. Extensional crack. Northwest-trending extensional crack where dam fill settled about $0.6 \mathrm{~m}$ ( $2 \mathrm{ft}$ ) and pulled away from concrete spillway and north abutment of Austrian Dam in the Santa Cruz Mountains, CA, USA. Photograph taken on October 17, 1989 by R.J. McLaughlin, U.S. Geology Survey.

As a second example, the Shin-Kang Dam was partially destroyed after Chi-Chi earthquake that caused the irregular ground movements and separation of most of the concrete blocks from the foundation rock, which consists of layers of mudstone, siltstone, and sandstone (Bureau 2003). Yet another example is the case of the Austrian Dam in Santa Clara County, California, which

\footnotetext{
1 Settlement-induced transverse fissures have been reported as early as the 1920 s for several dams around the world (Hsu 1981).

2 Earthquake-induced deformations and thus transverse fissures were reported recently in USCOLD (1992). Earthquake deformations may result in separation between the embankment and the adjacent concrete structure.
} 
suffered serious deformations, fissuring, and separation of the embankment from the spillway, occurring to a depth of $23 \mathrm{ft}$ on the right abutment. Water levels in the embankment, measured in piezometers, increased as a result of the 1989 Loma Prieta Earthquake (Figure 1).

The epicenter of the Loma Prieta Earthquake was seven miles from the Austrian Dam, a 200-ft high, 700-ft long embankment dam constructed in 1949-50 near the town of Los Gatos. The design called for an upstream impervious zone, a downstream pervious zone, and highly pervious strip drains located near the old stream channel in the downstream zone (USCOLD 1992). However, the weathered sedimentary rock at the site broke down during excavation, placement, and compaction, resulting in a nearly homogeneous gravelly, clayey sand embankment, compacted to approximately $90 \%$ of the maximum density. Most all soils and highly weathered rock were removed from the dam footprint prior to the embankment construction. The dam is in a v-shaped canyon in the Santa Cruz Mountains, about 2,000ft northeast of the San Andreas Fault Zone. The earthquake caused a maximum settlement of $2.8 \mathrm{ft}$, with significant settlement occurring over the right three-quarters of the dam (Rodda et al. 1990). Maximum downstream movement was $1.1 \mathrm{ft}$ near the spillway wall on the right abutment, and maximum upstream movement was $0.4 \mathrm{ft}$ at the left quarter point of the embankment. Longitudinal cracks up to $1 \mathrm{ft}$ wide and $14 \mathrm{ft}$ deep (see Figure 1 ) occurred within the upper $25 \%$ of the upstream and downstream faces. Shallower longitudinal cracks were found on much of the downstream face. Crest cracking was confined to the abutment contact areas (Figure 1 and Figure 2). Transverse cracking and embankment separation from the spillway structure occurred to a depth of $23 \mathrm{ft}$ and a maximum width of $10 \mathrm{in}$. (Figure 2). The separation was apparently due to a combination of soil structure interaction, embankment settlement along the very steep abutment and permanent wall deflection. A transverse crack was traced 30ft down the left abutment, where the dam had been constructed on weathered, highly fractured rock. The settlement and cracking at both ends of the dam crest was partially the result of a low density embankment that was rapidly placed to top out the dam after the start of the 1950 rainy season.

The phenomenology leading to failure after cracking is an elevated seepage flow which can cause further degradation and erosion of the dam core. Filter zones are engineered to capture core particles and prevent catastrophic failures. Unfortunately, if cracking is too extensive, filters, which are designed for certain range of operating conditions using empirical approach, may not be capable to handle the incident. Internal erosion of embankment dams under normal conditions is defined as a process of washout of fine-grained particles from a dam's filling material by water seepage as it flows through cracks, gaps at interfaces between embankment sections or their foundations, or other preferential flow paths. If the crack continues to grow, it may lead to the complete collapse of the dam and release a major flood. 
To preclude such failures, dams' performances must be periodically evaluated for increased internal erosion and filtration.

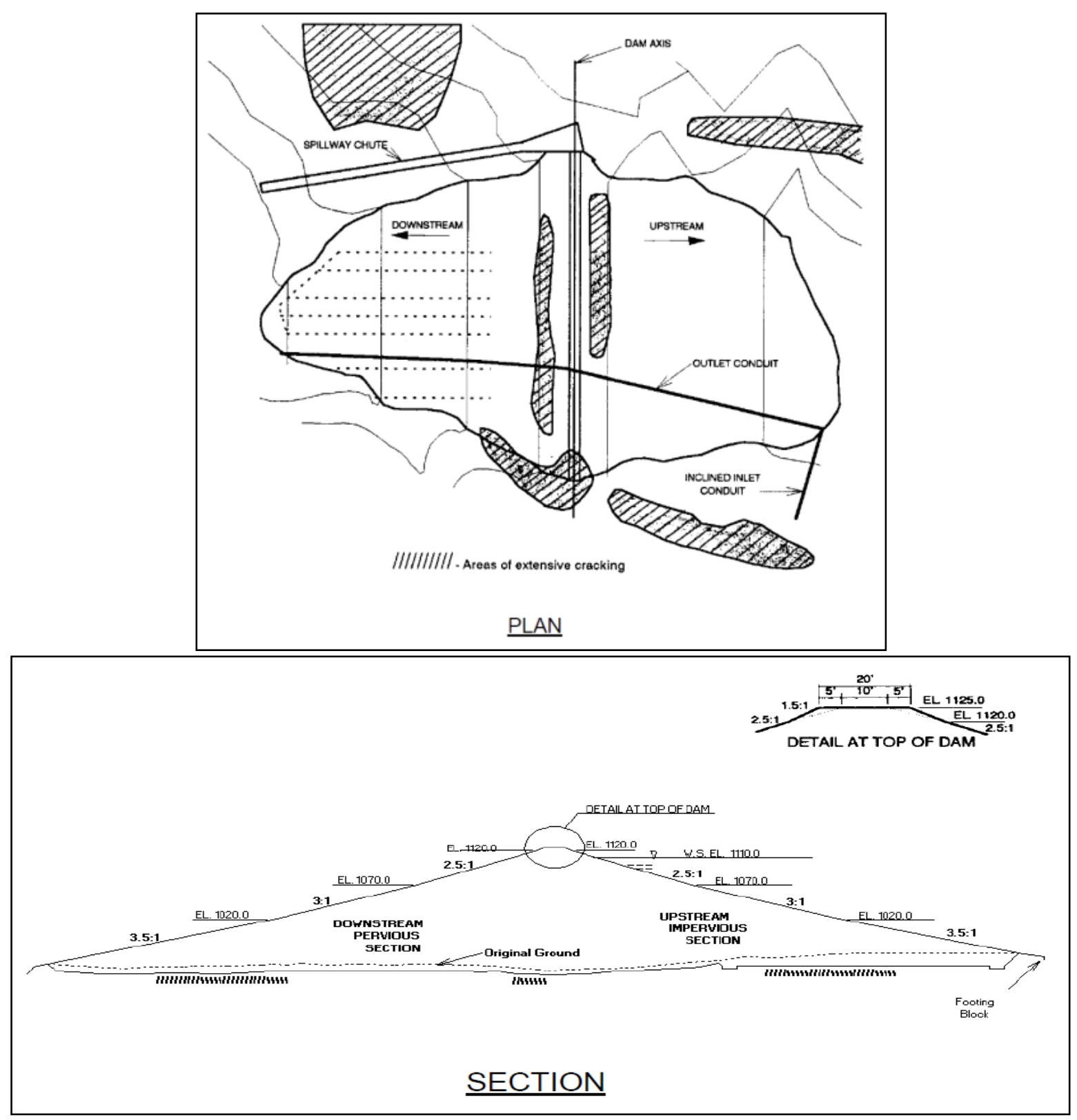

Figure 2. Austrian Dam (Santa Cruz, CA). [Top] aerial view, hashed areas represent damaged zones [Bottom] cross-section (Babbit, www.michigan.gov/documents/deq/deq-p2ca-caseismicpaper_281049_7.pdf).

Granular filters are one of the most important protective design elements of large, zoned embankment dams. Figure 3 shows a series of schematic descriptions of a typical concrete embankment dam, and subplot $\mathrm{c}$ of the figure forms the basis of all subsequent analyses, and Figure 4 is a photograph of Folsom dam under construction where the wrap-around section is evident. The standard way to prevent internal erosion is using adequate granular filters in the 
transition areas where important hydraulic gradients can occur. In case of cracking and erosion, if the filter is capable of retaining the eroded particles, then the crack will seal, and the dam safety will be ensured. The problem has two important components: (1) soil erosion in a crack, and (2) soil capturing in filter layers. Therefore, an accurate prediction of soil erosion rates and properties of filter layers is required to ensure dam safety.

To predict soil erosion it is necessary to estimate granular flux based on the fluid flow properties and particle bed properties. This is often accomplished using "erosion laws." A collection of erosion laws have been proposed in literature (Ouriemi et al. 2009). Some expressions are based on linear dependence between granular flux and shear stresses (Wan and Fell 2004, Bonelli and Brivos 2008), others assume power law dependence (Meyer-Peter and Muller 1948, Lobkovsky et al. 2008) with exponents close to 1.5 or 2 . Debates are still ongoing on the adequacy of those erosion laws which require further validation studies and deeper understanding of the combined fluid/granular (two-phase) flow. This work is an attempt toward explaining the differences between these erosion laws using validated numerical experiments.

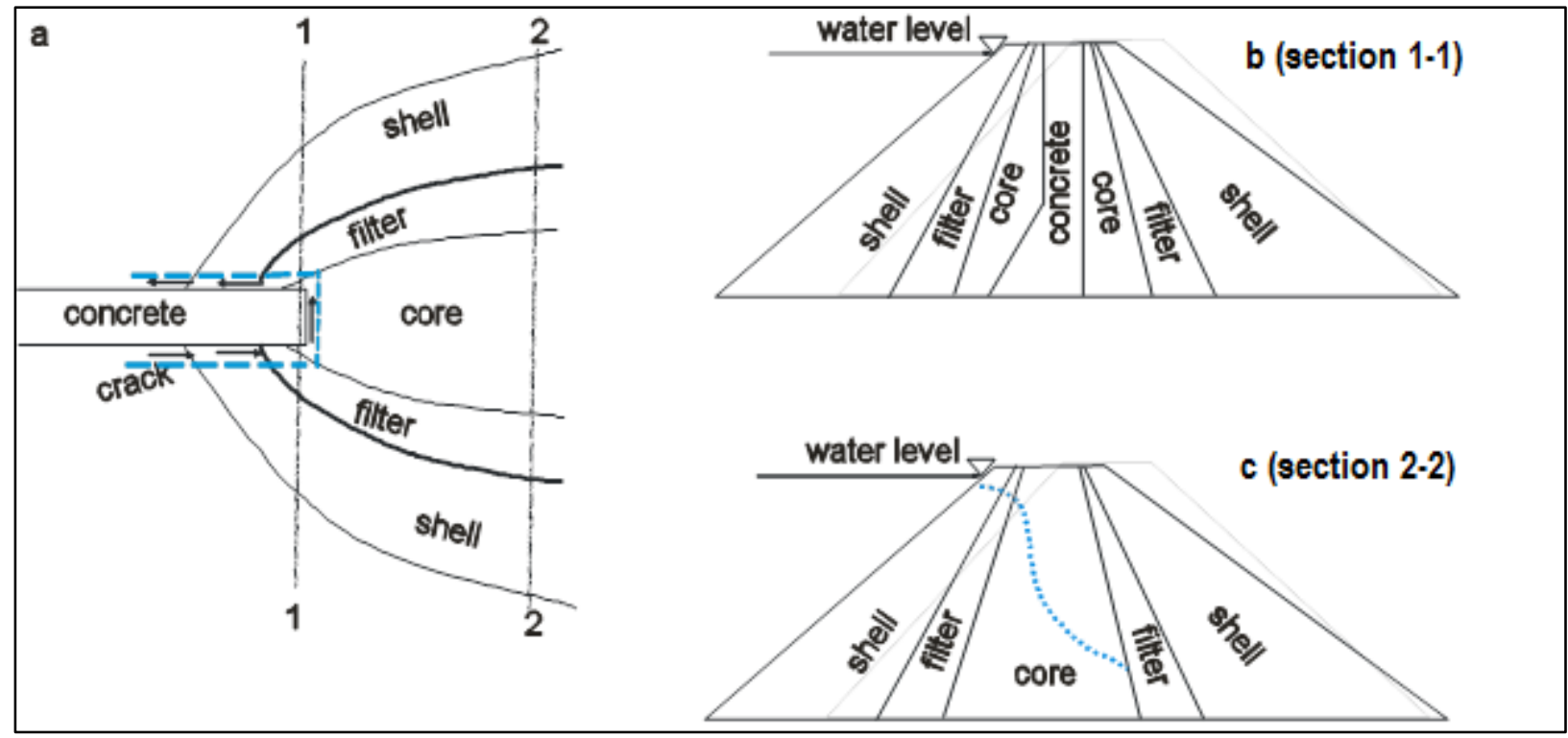

Figure 3. Schematic description of typical layers for concrete dam embankment. (a) Aerial view. (b), (c) side-slice views. Layers of embankment, water level, and phreatic line (the line corresponding to the phreatic surface lying above the saturated zone when seen in a vertical plane) are shown.

The objective of this joint effort by the Lawrence Livermore National Laboratories (LLNL) and the Engineer Research and Development Center (ERDC) of the US Army Corps of Engineers (USACE) is to develop numerical tools to predict the long-term behavior of the interface between a concrete gravity dam with an embankment zone referred to as the wrap-around section. The numerical tools required to accurately capture this complete phenomenology are 
at both dam and grain scale, which require several detailed experiments. Experiments were conducted at ERDC to provide accurate data and observations to assist in the verification and validation of the numerical models development at LLNL. The reduced dam scale experiments were conducted to understand the overall phenomenology at the interface between an embankment and a concrete gravity dam. The grain scale experiments provide detailed data on flow fields carrying fine grain material through a filter material. The data will quantify the filter zone's ability to stop the propagation of a crack between the interface of an embankment and concrete gravity dam.

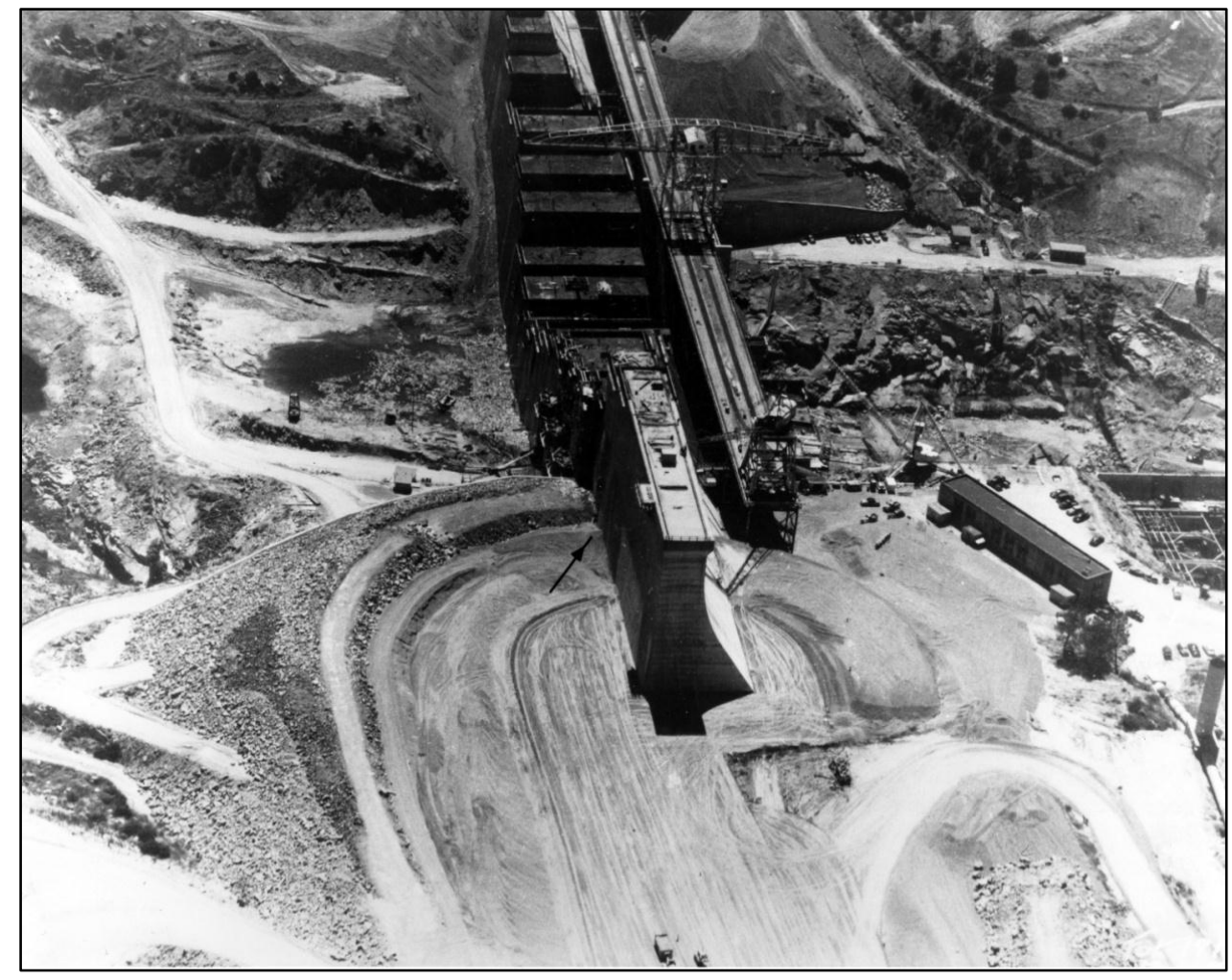

Figure 4. Construction of the wrap-around section of Folsom Dam

The objective of this joint effort by the Lawrence Livermore National Laboratories (LLNL) and the Engineer Research and Development Center (ERDC) of the US Army Corps of Engineers (USACE) is to develop numerical tools to predict the long-term behavior of the interface between a concrete gravity dam with an embankment zone referred to as the wrap-around section. The numerical tools required to accurately capture this complete phenomenology are at both dam and grain scale, which require several detailed experiments. Experiments were conducted at ERDC to provide accurate data and observations to assist in the verification and validation of the numerical models development at LLNL. The reduced dam scale experiments were conducted to understand the overall phenomenology at the interface between an embankment and a concrete gravity dam. The grain scale experiments provide detailed data on 
flow fields carrying fine grain material through a filter material. The data will quantify the filter zone's ability to stop the propagation of a crack between the interface of an embankment and concrete gravity dam.

\section{PROBLEM DESCRIPTION}

The complete computational space of this problem spans from accurate damage prediction from an extreme multi-hazard event that creates a crack or dislocation along with the soil erosion processes within the dislocation zone, to stability and collapse of the wrap-around. Relevant phenomena are inherently multiscale both in space and time: seismic events have a second-to-minute time scale, shock dynamic loading has a millisecond time scale, but catastrophic failure can happen from within a few hours to weeks or even months after a triggering event. There are also intermediate time scales due to sloped soil collapse (seconds), water seepage or flow (minutes), and soil/gravel erosion and deposition (hours to days). In terms of length-scale, the problem can be studied at the full dam scale, at the crack scale, or at the grain scale. Some of these approaches are extremely computationally intensive, requiring a large investment in computer resources and computational validation.

For this initial conceptualization discussed here, we focus on the existence of a specific dislocation between the concrete and embankment section and whether or not such dislocation or crack can remain open for sustained water flow or initiate an internal erosion process. Internal erosion of embankment dams under normal conditions is well described in literature (Fell et al. 2001). Internal erosion is defined as a process of washout of fine-grained particles from a dam's filling material by water seepage as it flows through cracks and gaps at interfaces between embankment sections or their foundations, or other preferential flow paths. Piping is among the most important causes of dam failure under normal operational conditions. It occurs when internal erosion due to water flow through poorly compacted soil or a crack in the embankment dam get localized over time to form a 'pipe.' If the pipe continues to grow, it may lead to the complete collapse of the dam and release a major flood.

According to Foster et al. (2000), about $46 \%$ of all dam failures can be attributed to some form of piping, and about $30.5 \%$ of all dam failures were due to piping through the embankment. For example, both Teton Dam and Baldwin Hills Dam failed due to piping in approximately four hours (Solava and Delatte 2003, Saxena and Sharma 2005). Design of existing dams varies widely depending on loading prehistory, material properties, and compatibility of materials. While internal erosion in embankment dams under normal operational conditions can be accurately monitored and prevented (e.g., Fell et al. 2001), the genesis of our work is primarily to help evaluate consequences of dynamic loading due to earthquakes or explosives at the wrap section of a gravity dam. In this case, the internal erosion can be a relatively rapid process, 
since dynamic loading can enhance crack formation and increase seepage flow rates. At this stage, we developed and evaluated numerical tools and capabilities to address different damage scenarios. We plan to tailor them for particular material properties and dam structure at later stages based on real field data.

\section{FAILURE CLASSIFICATION}

We can group damage severity to soil-concrete interfaces into three categories:

1. Structural damage to watertight core material with minimal damage to the concrete interface: Inelastic deformation could cause cracking in the core material and development of leakage paths, which may or may not lead to further erosion. The question to be answered is whether or not larger voids can develop, leading to instability. The associated soil deformation behavior and permeability change can be studied experimentally or with mesoscale simulations of a representative volume element (RVE) deformation of the core material.

2. Significant damage to the interface between the core material and concrete: Here there is no initial damage to the filters, but there is seepage flow at very low Reynolds number within the core section. The possibility of subsequent dam failure depends significantly on the filter design specifications. The filter can prevent further erosion if the core material fills the voids between filter grains and stop the flow. But it is also known that a "suffusion" process in coarsely graded filter material, i.e., when finer particles are eroded through the pore space between the larger particles, could lead to the filter failure. While the filter design criteria are in most cases empirically derived, numerical modeling can serve as complimentary approach for investigating filter stability.

3. Significant opening between the concrete and both filter and core sections. Large water flow through the gap can lead to high Reynolds number flows, which can easily carry large particles and cause instability and collapse of eroded slopes. This regime will lead to rapid erosion of both filter and core zones and systematic (catastrophic) failure. But even in this case, large gravel will have a high settling velocity and may occlude the lower portion of the crack, ultimately stabilizing the process. The filter and shell materials can be investigated experimentally and numerically to see if they fill the crack and prevent the erosion process from leading to failure.

From failure of a crack to self-healing of a crack is a gradual transition from a damage regime to an altered, but stable, condition. At one extreme, there is a light damage associated with unconnected shear induced voids. At the other extreme, there is degradation or healing of moderately to severely damaged regions that can be linked to fluid flow, sliding of materials 
into the cracks, and possible erosion and downstream deposition of clay, sand, or gravel. Removal of relatively large volumes of earthen materials can alter stress state in a dam and eventually may lead to its collapse due to slope instability. Also, cracks forming through the core could result in the downstream zone becoming saturated, which could also contribute to slope instability.

\section{PROPOSED WORK}

In the current study, we approach the problem through integration of experimental investigations with the numerical simulation of the physical processes that take place at the grain scale and the dam scale. At the grain scale, we numerically represent the flow physical process using two fundamentally different approaches. The first uses an Eulerian-Eulerian multiphase formulation, where fluid and particulate phases with different particle sizes are represented by corresponding mass fractions. This approach is most suitable when considering an entire system, but requires computationally demanding closure laws for interphase momentum exchange. The second approach explicitly resolves the volume occupied by solid particles and is applicable for densely packed particle beds. This second approach limits the number of necessary parameterizations and is often used to help parameterize the more computationally intensive Eulerian-Eulerian approach. Due to the large variation in particle size (from microns to centimeters), both approaches have to be used in a single simulation to evaluate the ability of the filter zones (with resolved particles) to capture the unresolved finer particles. At the dam scale, we numerically simulate the flow and transport of fines process using two different Eulerian approaches. At first, the flow and transport of fines obey singlepopulation advection-dispersion with linear and non-linear mobilization and immobilization of fines. We then improve the flow and transport of fines using two-population advectiondispersion model, where both mobile and immobile fines are tracked through their time evolution across the embankment using linear and non-linear attachment and detachment kinetics of the fines. Throughout the different investigations, numerical simulations are compared to experiments whenever possible and whenever experiments are available.

\section{EXPERIMENTAL INVESTIGATIONS}

\section{DAM SCALE EXPERIMENTS}

Two experiments were conducted using a 13-in. high concrete gravity dam with an 8-in. base embedded into a zoned embankment. The impervious core of the zoned embankment was Vicksburg silt, taken from a site on the ERDC Vicksburg installation. Vicksburg silt is a windblown silt deposit that is typically brown, with traces of sand. The general soil properties for the Vicksburg silt determined from laboratory tests are as follows: 
- Liquid Limit: LL = 43 to 34 (from "Advanced Triaxial Testing of Soil and Rock," Symposium 19-20 June 1986)

- Plastic Limit: PL = 22 to 13 (from "Advanced Triaxial Testing of Soil and Rock," Symposium 19-20 June 1986)

- Minimum Particle Size: $20 \%$ passing a $0.005 \mathrm{~mm}$ sieve

- Maximum Dry Density: 110 pcf (@ Standard Proctor)

- Optimum Moisture Content: $15 \%$

- Specific Gravity: 2.68

- Density: $92 \mathrm{lbs} / \mathrm{ft} 3$

A 70/30 gravel and silt mix was used for the shell of the zoned earthen embankment model. It was mixed in a similar manner, in the proportions of $70 \%$ gravel and $30 \%$ silt, by weight. The core dimension was $12 \mathrm{in.} \mathrm{high,} 20 \mathrm{in}$. long, and $11 \mathrm{in.}$ wide (container side), 5 in. wide (dam side). The transition zone was $12 \mathrm{in.} \mathrm{high,} 22 \mathrm{in.} \mathrm{long,} \mathrm{and} 15 \mathrm{in}$. wide (container side), $8.5 \mathrm{in.}$ wide (dam side). The outer embankment slope was 1:2.5.

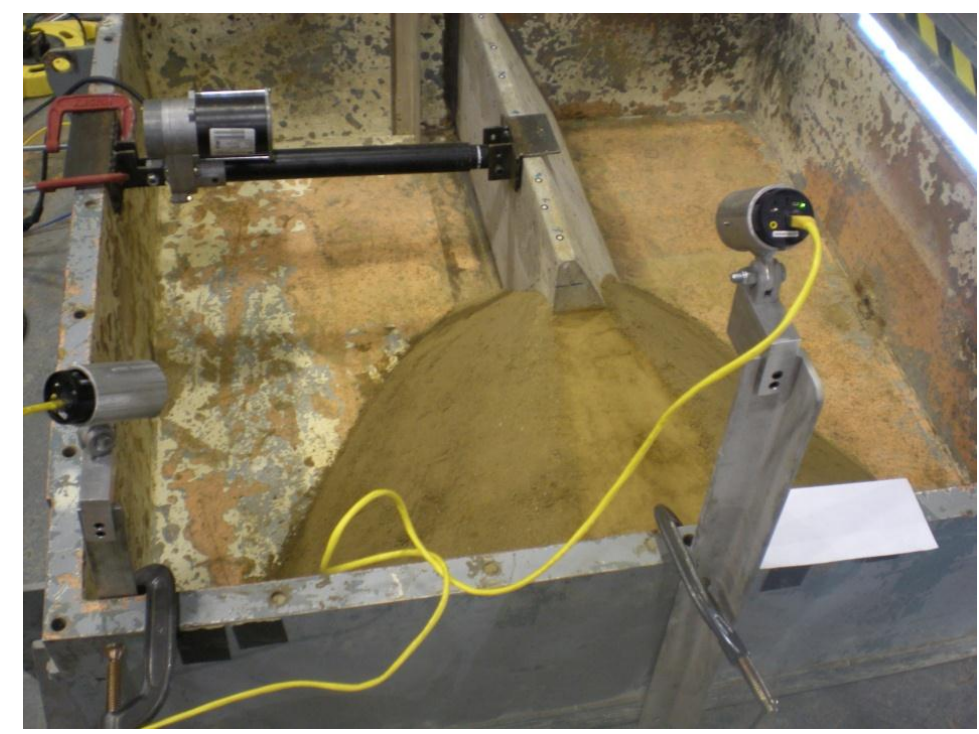

Figure 5. Test set up for embankment with silt core and transition zones.

\section{EXPERIMENTS AT $1 G$}

The first experiment was conducted at $1 \mathrm{~g}$. A displacement actuator was placed on the crest of the dam, resulting in rotation of the concrete section about the toe in the downstream direction (Figure 5). The model container was placed on the laboratory floor near a drain to allow the removal of any downstream water. Water was then filled to the desired elevation (top of embankment with no overtopping) and allowed to saturate for thirty minutes. The actuator pushed the top of the dam approximately $1 / 2$ in. every 15 minutes. The 15 minutes 
allowed any seepage to occur resulting from cracks in the embankment section of the dam. As the test progressed with a crest displacement of $1 \frac{1}{4}$ in., a crack in the embankment section at approximately 45 degrees from the plane surface of the concrete monolith grew to approximately 6 in. At this time, a small amount of seepage was seen on the landside at the toe and at the tip of a crack (Figure 6).

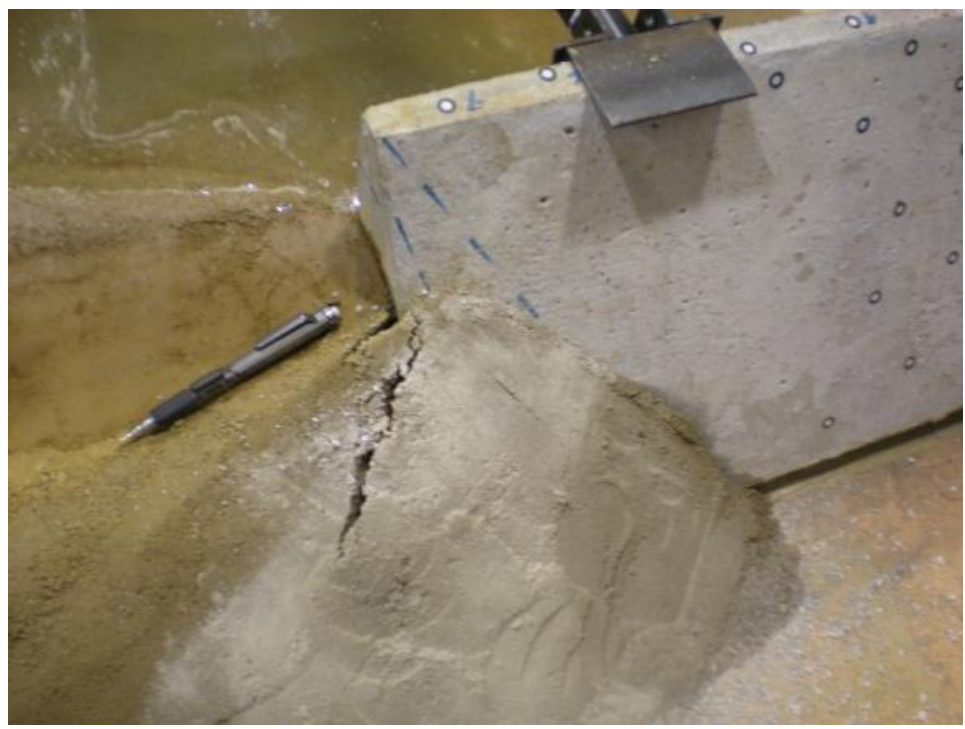

Figure 6. Indication of seepage at tip of crack at the upper section and at toe.

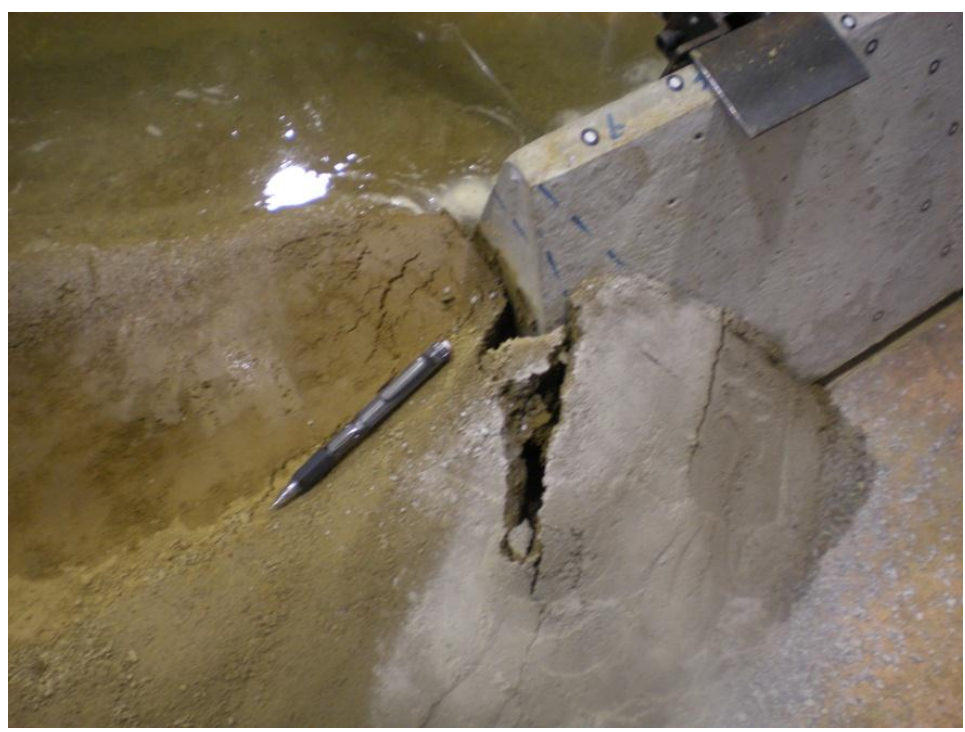

Figure 7. Downstream cracks and drying wet spot at toe, no flow through.

These wet spots did not continue to grow, but started to dry slightly with further displacements of the dam crest. This drying action indicated that the filter material combined with material falling into the upstream cracks reduced the seepage through the damaged section (Figure 7). The model dam did not lose the reservoir with a rotation of the concrete section at a horizontal 
crest displace of 4.125 in. The significant rotation resulted in a horizontal crack forming at the tip of the initial 45 degree crack. This horizontal crack resulted in a complete wedge of the embankment displacing downstream. At this point, the seals preventing seepage beneath the concrete section failed, ending the experiment. Some seepage did occur along the horizontal crack (Figure 8).

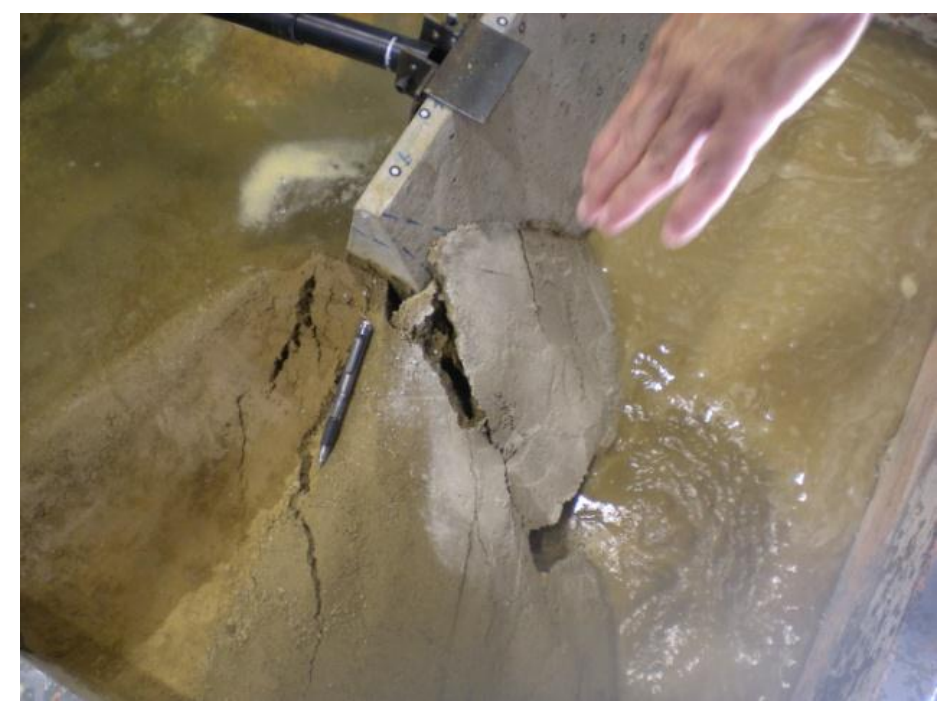

Figure 8. Seepage through horizontal crack and base of concrete section.

\section{EXPERIMENTS AT 30G}

The second experiment was performed on the centrifuge. This experimental dam model was identical to the initial experimental model with added outer shell zone of $100 \%$ crushed gravel and was subjected to increased gravitational loads provided by the centrifuge (Figure 9).

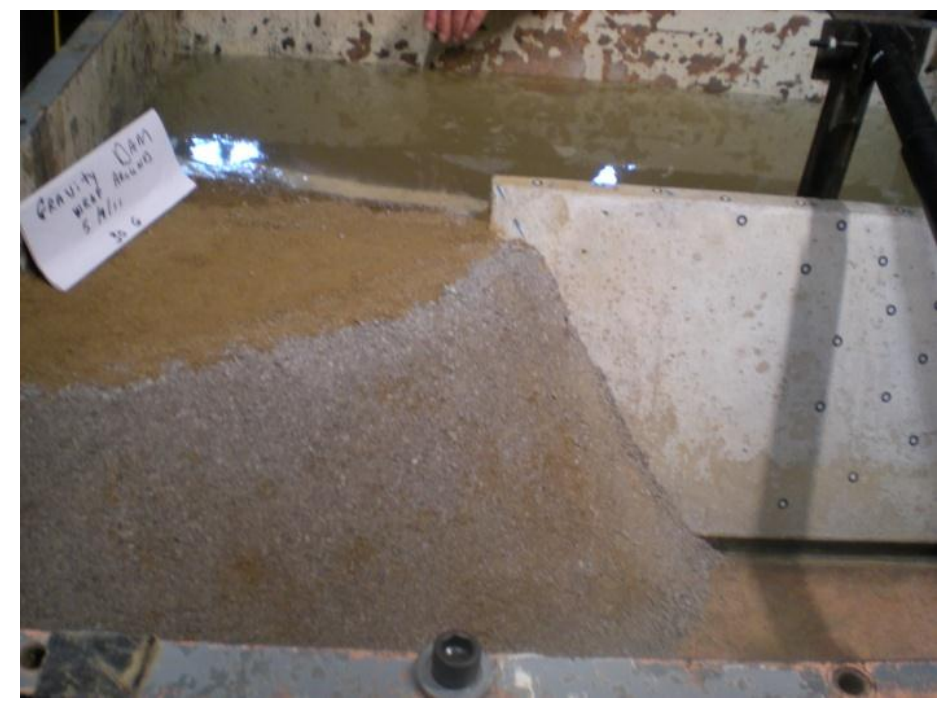

Figure 9. Centrifuge model. 
The crushed gravel was added to highlight seepage occurring through the embankment. The displacement actuator was attached to a rigid link at the bottom of the concrete gravity section. This displacement actuator resulted in a rotation of the concrete gravity section about the downstream toe of the section.

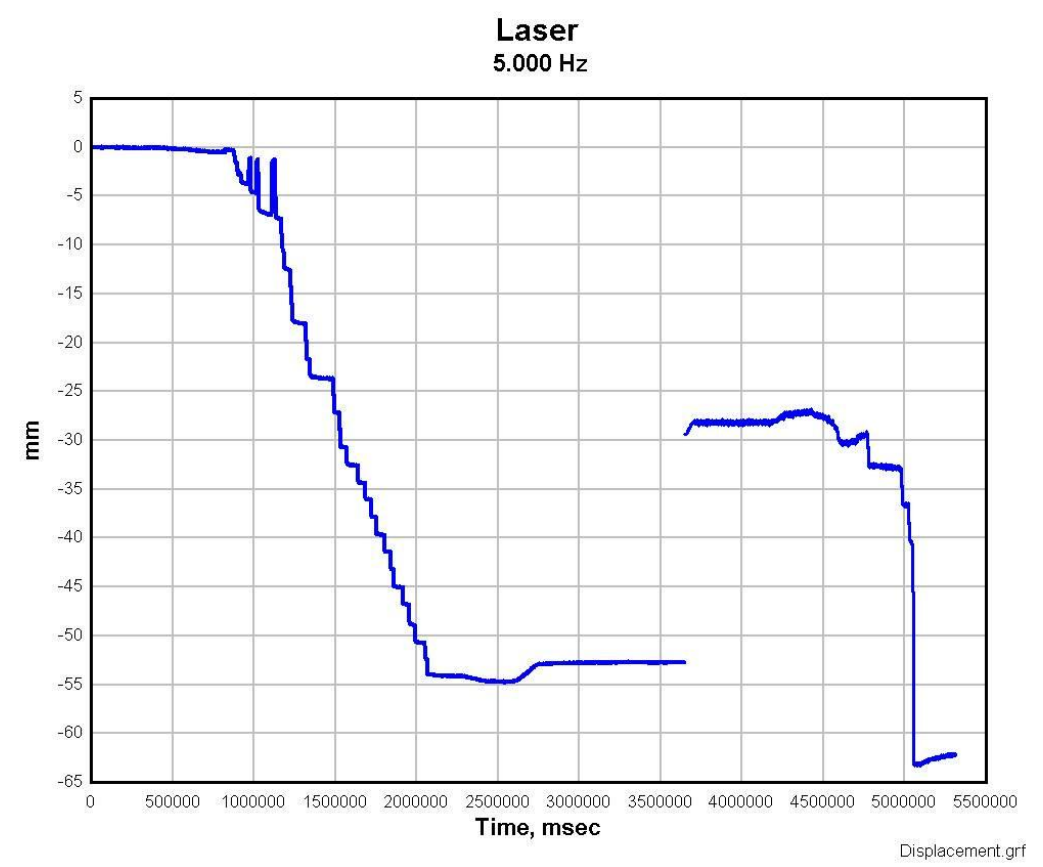

Figure 10. Displacement plot of crest of concrete gravity section.

The addition of gravity was to represent a full-scale dam and to determine if the failure mechanism from the $1 \mathrm{~g}$ experiment would scale to a full size dam. The displacement actuator was tested on the centrifuge before this experiment to ensure its performance for this experiment. Modification of the actuator was required to reach the desired length of "push." The functionality of the actuator was limited to gravity forces of $30 \mathrm{~g}$ 's. The centrifuge model was filled prior to flight to allow for saturation and to ensure the centrifuge was not out of balance. In-flight water was added to maintain the desired elevation to replace the amount of seepage into the embankment. The model was subjected to $30 \mathrm{~g}^{\prime} \mathrm{s}$ and then was incrementally "pushed" 3-5 mm at 10 minute intervals. The horizontal displacement was measured by a laser displacement gage in flight. The displacement actuator reached its stroke limit of $53 \mathrm{~mm} \mathrm{(2.1}$ in.) after $35 \mathrm{~min}$ (Figure 10).

At this point the centrifuge was allowed to spin down. The arm of the actuator was lengthened in order to provide additional stroke. The model was again subjected to $30 \mathrm{~g}$ 's. Some rebound occurred due to the tilt of the arm of the displacement actuator at the second start. Multiple cracks in the embankment were observed on the downstream section of the embankment section of the model. The dominant crack was approximately 45 degrees from the plane surface 
of the concrete monolith, just as in the previous $1 \mathrm{~g}$ experiment (Figure 11). However, no seepage occurred through the damaged embankment section. At a crest displacement of 63.3 $\mathrm{mm}$ ( $2.5 \mathrm{in}$.) the seal at the bottom of the concrete gravity section broke, resulting in the loss of the model reservoir. The residual displacement of the crest of the concrete gravity section, measured at $1 \mathrm{~g}$, was $1.75 \mathrm{in}$. Once the seal on the bottom of the concrete section failed, the concrete gravity section slid, reducing the rotation of the concrete gravity section and the corresponding displacement of the crest.

The failure modes of this embankment/concrete gravity dam model at $1 \mathrm{~g}$ and $30 \mathrm{~g}$ 's were almost identical. Both models had a dominate crack 45 degrees from the plane surface of the concrete monolith, forming a wedge of material that moved downstream in the $1 \mathrm{~g}$ experiment. The $30 \mathrm{~g}$ experiment had less rotation and correspondingly less displacement (maximum crest displacement at $1 \mathrm{~g}$ was 4.124 in., and at $30 \mathrm{~g}^{\prime} \mathrm{s}, 2.1 \mathrm{in}$.) resulting in the wedge of the downstream embankment material not moving and forming the horizontal crack. Both models had significant cracking with no seepage occurring through the damaged embankment.

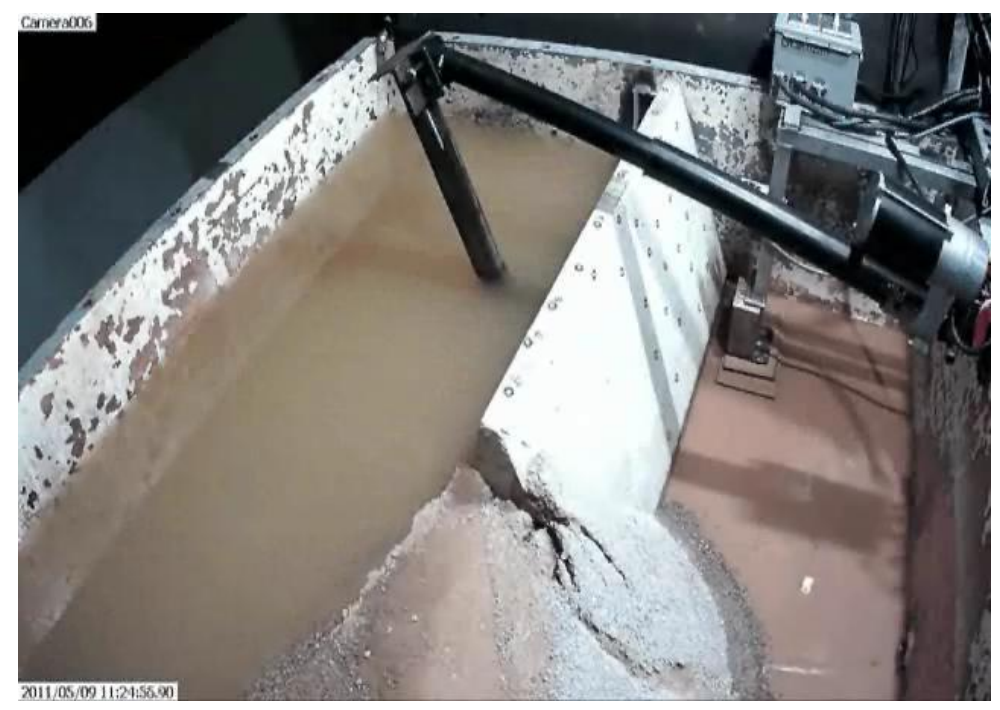

Figure 11. Downstream cracks at crest displacement of $2.1 \mathrm{in.}$

\section{GRAIN SCALE EXPERIMENTS}

This section of the report describes activity towards measuring transport and trapping of fine sediment in granular filter media. These laboratory experiments are designed to produce data for evaluation of LLNL direct numerical simulations of fine sediment transport through filter media. Currently, the direct numerical simulations are performed with spherical particle assumptions (recently the LLNL modeling team has added the capability to model non-spherical particles). Laboratory experiments by the subject research will be conducted with both spherical and irregular (natural) media and transportable particles. The objective of these 
experiments is to provide data for the LLNL modeling team to evaluate fine particle transport within filter media and the resulting trapping rates and efficiency of various combinations of fine soil particles and filter media.

\section{APPROACH}

The experiments will be conducted with a constant-head flow chamber filled with filter media. A suspension of fine particles will be passed through the filter medium and imaging of the flow paths, velocity, and media interactions will be recorded with high-magnification, highresolution videography. During the experiments, samples will be drawn from the inflow and outflow to determine time-variant filter efficiency of the media. At the conclusion of an experiment, the filter medium will be sampled to determine mass and size distributions of the trapped particles.

\section{METHODS}

\section{CONSTANT-HEAD FLOW CHAMBER}

The constant head flow chamber (Figure 12) is designed to constrain the filter media and pass flow of a fine-soil suspension through the media.

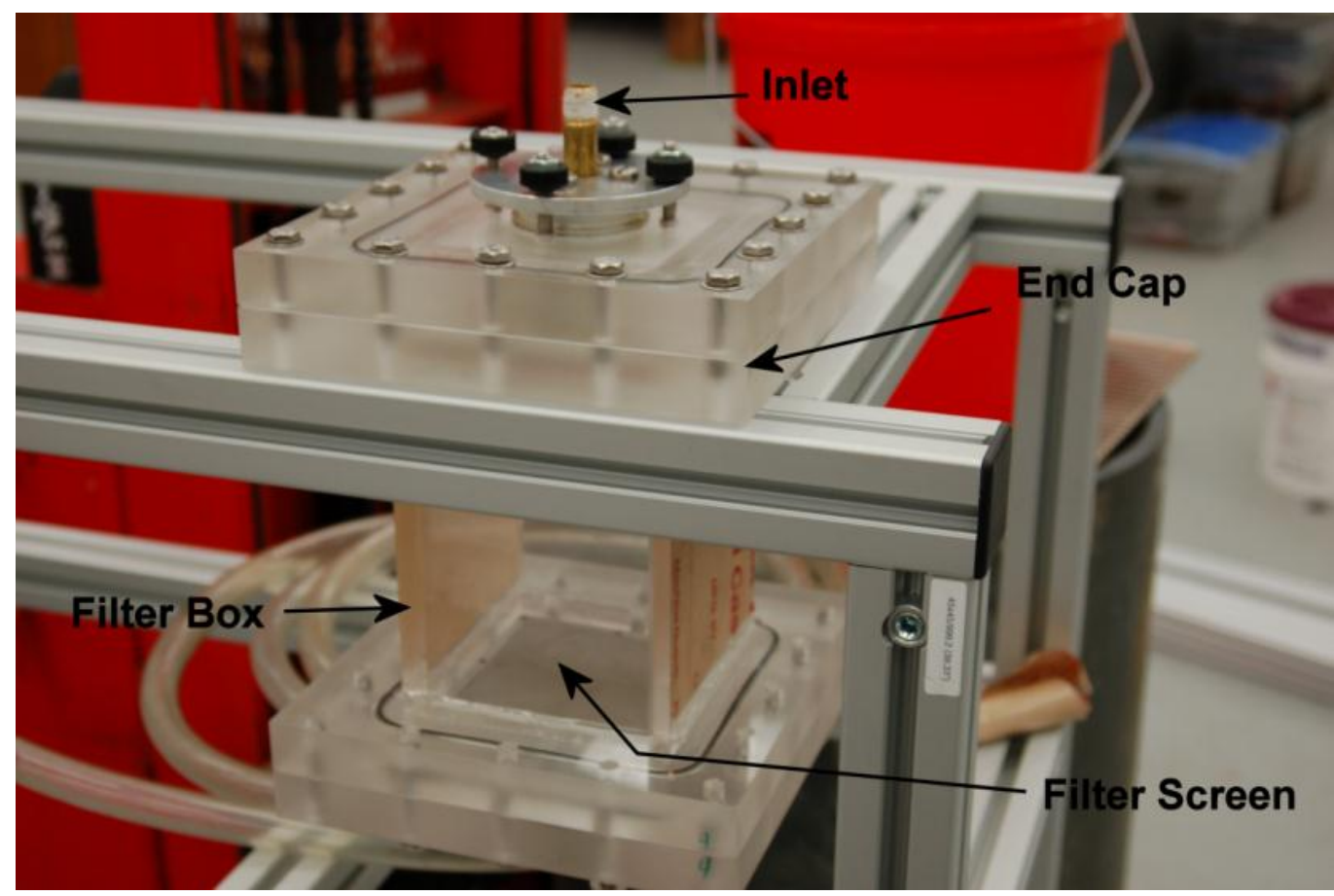

Figure 12. Constant head flow chamber, mounted in testing frame. Chamber cross-section is $10 \mathrm{~cm} \mathrm{x}$ $10 \mathrm{~cm}$ and $20 \mathrm{~cm}$ in length. 
The flow chamber is constructed of acrylic sheeting with inside dimensions of $10 \times 10 \times 20 \mathrm{~cm}$ $(\mathrm{W} \times \mathrm{DxL})$. Each end of the flow chamber has an end cap with 1/4 -inch plumbing for passing the fine-particle suspension through the media. Each end cap includes a screen (to retain the filter media) which rests upon a flow distribution plate that ensures uniform delivery of the flow across the cross-section (Figure 12).

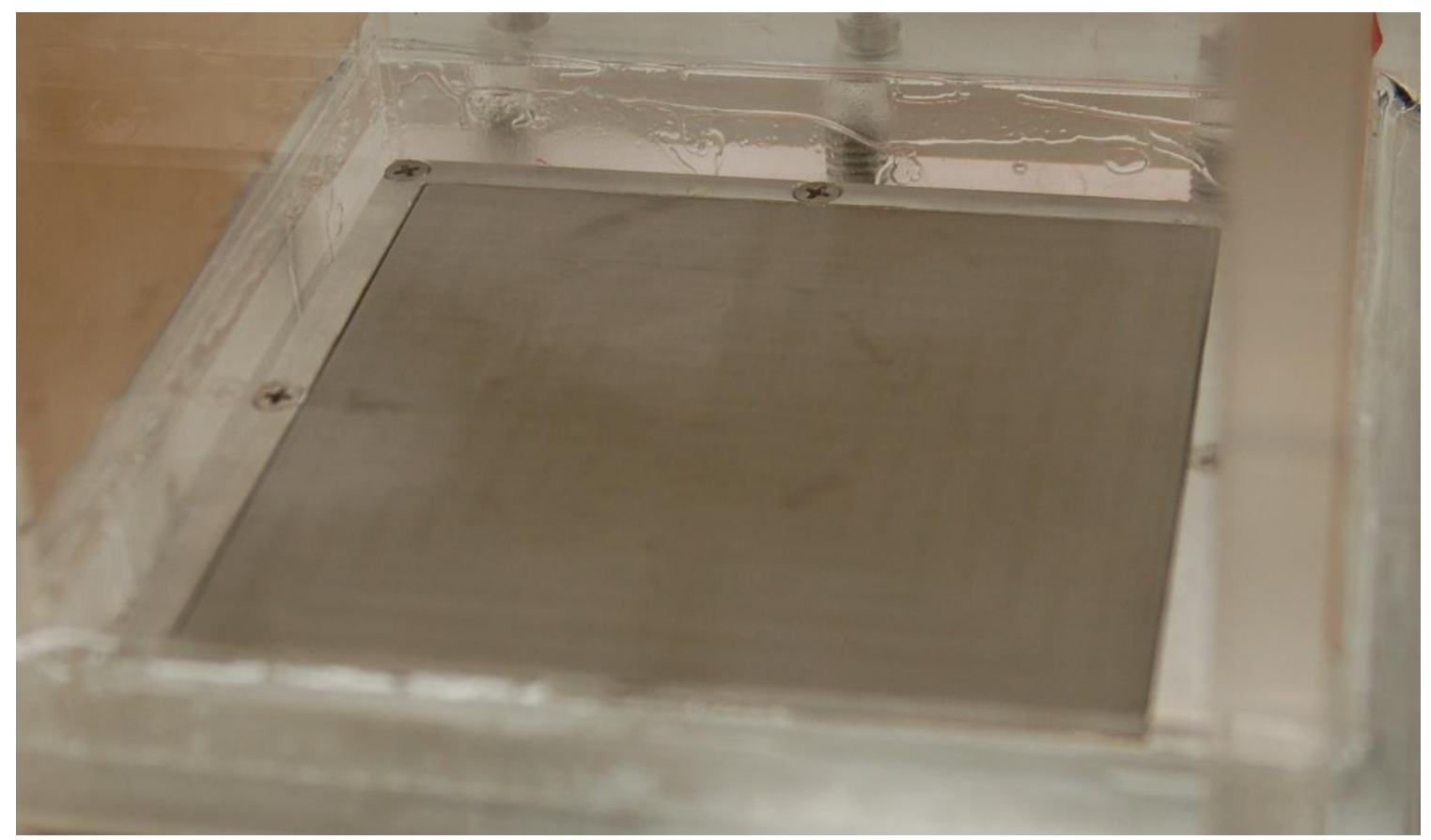

Figure 13. Filter screen for retaining filter media. Also visible is the flow distribution plate, which provides flow pathways between the inlet and outlet tubing and the filter media.

Flow is delivered to the flow chamber from a constant head tank (Figure 14), which is supplied with flow by a pump in the supply reservoir. Constant head is maintained by means of a vertical riser (2-inch PVC) which permits any excess pump flow to return to the supply reservoir. The total head across the flow chamber may be adjusted by raising or lowering the head tank with the hydraulic lift. Also shown in Figure 14 is a degassing system, implemented to prevent gas precipitation on the filter media (a problem encountered during initial testing).

FILTER MEDIA AND Transportable PARTICLES

Filter media and fine particles for evaluation in the experiments are presented in Table 1. Particle sizes for the spherical soda-lime glass beads are as inferred from the manufacturer's reference samples, actual sizes and properties will be determined during the study. 
Table 1. Materials for filter media and transportable particles in experiments.

\begin{tabular}{|llccccc|}
\hline Material & Shape & $\begin{array}{c}\text { Use } \\
\text { Media, } \\
\text { Transport }\end{array}$ & $\begin{array}{c}\text { Size Range } \\
(\mu \mathrm{m})\end{array}$ & $\begin{array}{c}\text { D15 } \\
(\mu \mathrm{m})\end{array}$ & $\begin{array}{c}\text { D85 } \\
(\mu \mathrm{m})\end{array}$ & $\left(\mathrm{g} / \mathrm{cm}^{3}\right)$ \\
\hline Soda lime glass & Spherical & $\mathrm{T}$ & $40-70$ & 40 & 70 & 2.50 \\
\hline Soda lime glass & Spherical & $\mathrm{M}, \mathrm{T}$ & $300-400$ & 325 & 375 & 2.50 \\
\hline Soda lime glass & Spherical & $\mathrm{M}$ & $1300-1700$ & 1300 & 1700 & 2.50 \\
\hline Quartz & Irregular & $\mathrm{M}, \mathrm{T}$ & $100-250$ & - & - & 2.65 \\
\hline Quartz & Irregular & $\mathrm{M}, \mathrm{T}$ & $250-500$ & - & - & 2.65 \\
\hline Quartz & Irregular & $\mathrm{M}$ & $500-1000$ & - & - & 2.65 \\
\hline Quartz & Irregular & $\mathrm{M}$ & $1000-2000$ & - & - & 2.65 \\
\hline Vicksburg loess & Irregular & $\mathrm{T}$ & $5-57$ & 10 & 42 & 2.65 \\
\hline
\end{tabular}

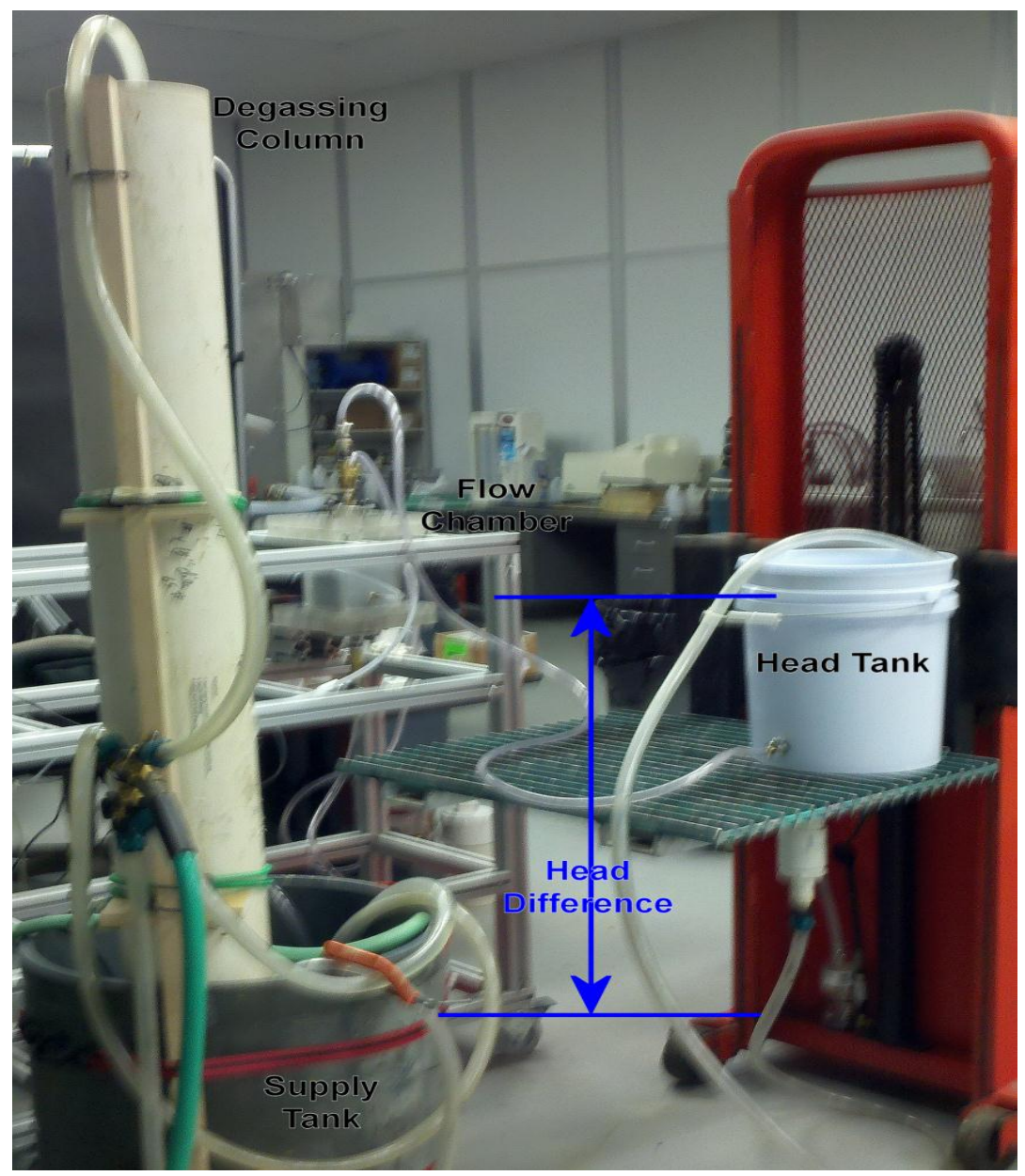

Figure 14. Experimental layout. Pump in supply tank provides flow to head tank and degassing column. Flow from head tank travels through tubing, through flow chamber, and back to supply tank. Head difference between head tank and supply tank drives flow through flow chamber. Hydraulic lift is used to adjust head difference (range 0.2 to $2.0 \mathrm{~m}$ ). 


\section{PARTICLE FLOW PATHS AND VELOCITY}

Advances in high-speed digital video cameras and video processing have increased the application of imaging methods to investigate fluid and fine particulate physics. This research will apply high magnification digital videography and video analysis to quantify fine particulate flow through granular media. The imaging aspects of the experimentation are constrained by optical limits, field of view considerations, and digital camera resolution. For fine soil particles in the size range of 10-60 microns (fine to coarse silt) a minimum field of view on the order of $4.5 \times 3.5 \mathrm{~mm}$ is possible. Particle Tracking Velocimetry (PTV) image analysis methods will be employed to quantify small particle velocities and trajectories through the filter media. Due to the limited depth of view for this imagery, the flow paths will be determined near the boundary between the media and the flow chamber wall.

FINE SOIL TRAPPING

Several techniques will be employed to quantify trapping capacity of the filter media for a range of fine-soil particle sizes. First, discrete samples will be taken from the inlet and outlet lines of the flow chamber at regular intervals over the course of each experiment. From these samples, flow rate, particulate concentration, and particulate size distribution will be determined. The inlet and outlet concentrations will be used to determine time-variant trapping efficiency of the filter media:

$$
\eta_{\text {filter }}=\frac{C_{\text {in }}-C_{\text {out }}}{C_{\text {in }}}
$$

where $C_{\text {in }}$ is inlet concentration, $C_{\text {out }}$ is outlet concentration. Size distribution of the filter media and fine-sediment will be conducted with standard laboratory methods including sieve analysis (ASTM D6913) and laser diffraction particle sizing (with a Malvern laser particle sizer). During the experiment, differential pressure across the flow chamber will be logged with an OmegaDyne differential pressure sensor at $10 \mathrm{~Hz}$. The measured pressure gradients and flow rates will allow an estimate of time-variant hydraulic conductivity:

$$
K=\frac{Q}{A \frac{d h}{d z}}
$$


where $Q$ is volumetric flow rate, $A$ is cross-sectional area of the flow chamber, and $d h / d z$ is the head gradient across the flow chamber. At the conclusion of the experiment, the flow chamber will be sub-sampled to quantify fine soil content within the filter media. Sub-sampling of the filter media will be attempted to quantify zones of trapping, both laterally and longitudinally within the flow chamber. Methods such as scooping or freezing and sectioning the sample will be attempted during initial testing and performed consistently throughout the laboratory experiments.

\section{PROPOSED EXPERIMENTS}

A listing of proposed experiments is provided in Table 2. This initial set of experiments is subject to modification as the research progresses and insights are gained. The first set of experiments will be conducted with spherical media and flow particles (fines).

Table 2. Proposed experimental combinations.

\begin{tabular}{|c|c|c|c|}
\hline $\begin{array}{l}\text { Media } \\
\text { (material, } \\
\text { nom. size } \mu \mathrm{m} \text { ) }\end{array}$ & $\begin{array}{l}\text { Fines } \\
\text { (material, } \\
\text { nom. size } \mu \mathrm{m} \text { ) }\end{array}$ & $\begin{array}{l}\text { Pressure } \\
\text { Gradient } \\
(\mathrm{m} / \mathrm{m})\end{array}$ & D15/d85 \\
\hline \multicolumn{4}{|c|}{ Spherical Experiments } \\
\hline SLG, 1500 & SLG, 40 & $0.1,0.5,1.0$ & 19 \\
\hline SLG, 1500 & SLG, 300 & $0.1,0.5,1.0$ & 3.7 \\
\hline SLG, 300 & SLG, 40 & $0.1,0.5,1.0$ & 4.6 \\
\hline SLG, 300 & Silt, 30 & $0.1,0.5,1.0$ & 7.7 \\
\hline \multicolumn{4}{|c|}{ Non-Spherical Experiments } \\
\hline Quartz, 1000 & Quartz, 250 & $0.1,0.5,1.0$ & 2.3 \\
\hline Quartz, 1000 & Quartz, 100 & $0.1,0.5,1.0$ & 4.9 \\
\hline Quartz, 1000 & Silt, 30 & $0.1,0.5,1.0$ & 26 \\
\hline Quartz, 500 & Quartz, 100 & $0.1,0.5,1.0$ & 2.4 \\
\hline Quartz, 500 & Silt, 30 & $0.1,0.5,1.0$ & 13 \\
\hline Quartz, 250 & Silt, 30 & $0.1,0.5,1.0$ & 6.3 \\
\hline Quartz, 100 & Silt, 30 & $0.1,0.5,1.0$ & 2.7 \\
\hline
\end{tabular}

\section{FINE-SOIL TRANSPORT AND TRAPPING IN GRANULAR FILTER MEDIA}

The purpose of this stage of the research is to evaluate experimental design and to resolve issues in the experimental materials and procedures. Initial testing of the experimental methods was started in March 2011. Presently the initial testing phase is nearly complete. This appendix provides results to date. This appendix will be amended in a future progress report to 
reflect completion of this task. An important aspect of the experimental procedures is maintaining constant hydrodynamic forcing and sediment loading across the media within the flow chamber. The experimental design requires a constant, reproducible head difference across the flow chamber. The head tank and pumping system was found to provide a stable, constant head over the range of experimental conditions.

During the initial testing, gas trapping within the media was a noted problem. Not only will this gas interfere with imaging, but it also generates a flow obstruction within the media that impacts fluid and particle motion. Two sources of gas trapping were noted. The first source is during filling of the flow chamber. If the chamber is filled too quickly or from the top, gas becomes trapped in the media. This problem was resolved by slowly filling the media from the bottom, allowing gas to be expelled from the media as the saturation level slowly rises within the chamber. The second source of gas entrapment was found to be associated with gas coming out of solution and precipitating on the media. After starting an experiment with gasfree media, small bubbles would form on the surfaces of the media (Figure 15A).
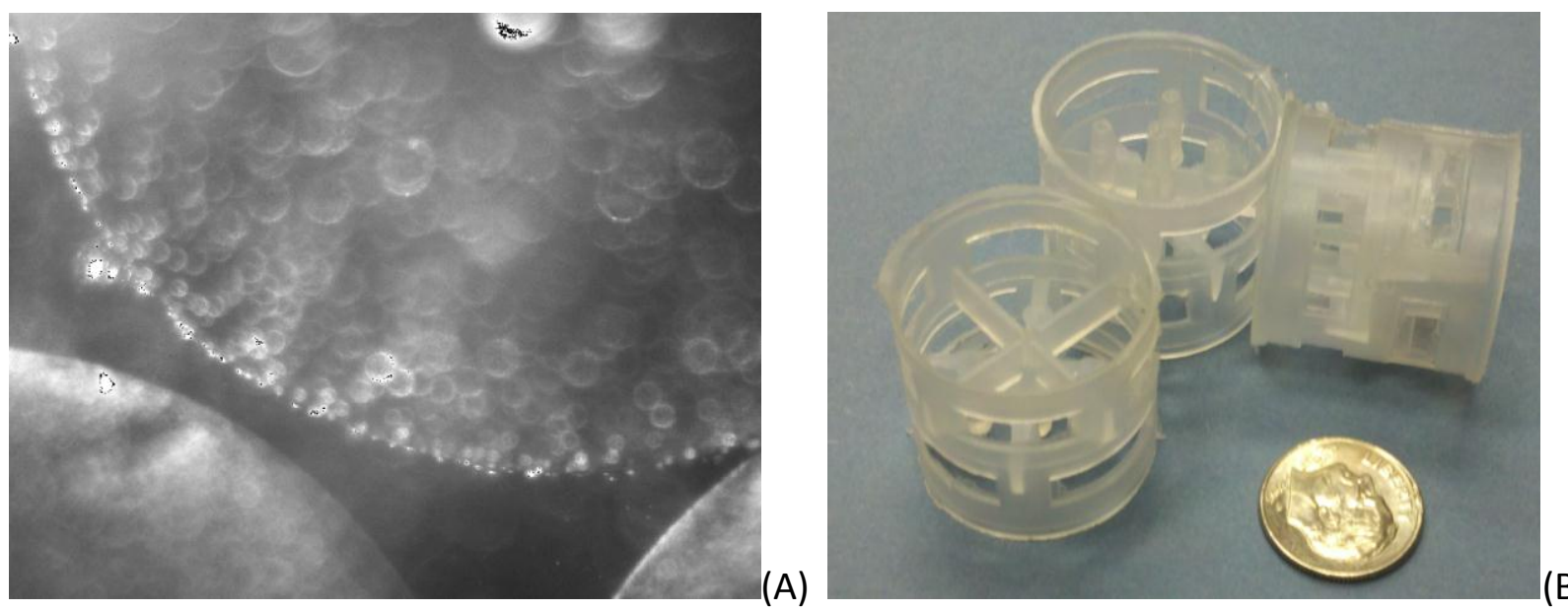

(B)

Figure 15. A) Gas bubbles that have precipitated from solution onto glass marbles used in initial testing experiments. B) Bio-barrels which provide a large surface area for degassing water within the degassing column.

Over time, these bubbles grew in size and aggregated. This degassing is related to the high gas content of tap water and the temperature rise of the water as it is recirculated through the system by the pump. Two solutions to the degassing issue were evaluated. First, high-intensity sonification was effective in removing virtually all dissolved gas from solution. However, the volume of water associated with the subject experiment is large relative to the processing rate with sonification, which would result in significant delays in experimental time. An alternate solution is a degassing column. Degassing columns are widely used by fish hatcheries to relieve supersaturated conditions. A small degassing column was designed and filled with 
commercially available, 1-inch diameter bio-barrels (Figure 15B). The degassing column is a 4-ft long, 6-inch diameter PVC pipe, packed with bio-barrels, which are retained at each end of the pipe with filter screens.

The column is oriented vertically and operated by pumping water to the top and allowing this water to trickle through the bio-barrels. The large surface area and thin sheet flow allows efficient gas exchange between the water and atmosphere. The degassing column is operated at full flow for 10 minutes prior to start of an experiment, and continually (at lower flow) during the experiment to relieve gas super-saturation. The operation of the degassing column has resolved all gas precipitation issues on the filter media. Trajectories and speed of fine particles passing through filter media are a primary objective of this research. These parameters will be measured through imaging and image analysis techniques. A fundamental requirement of this research element is to obtain high-quality images with appropriate temporal and spatial resolution for the scales of motion involved. A series of testing experiments were performed to evaluate available camera, optical, and lighting components.

CAMERA AND OPTICS

The ERDC Sediment Transport Laboratory currently uses several Gigabit Ethernet (GigE) cameras as part of ongoing sediment transport research. The Allied Vision/Prosilica GE2040 and GE1380 were evaluated to determine if these cameras would meet the requirements of the present study. The GE2040 is a 12-bit, $2048 \times 2048$ (4 MP) camera that can acquire images up to $15 \mathrm{fps}$. The GE1380 is a 12-bit, $1024 \times 1380$ (1.4 MP) camera with a maximum frame rate of 20 $\mathrm{fps}$, and excellent low-light sensitivity. When paired with the Navitar 3000 macro zoom lens, the field of view is approximately $6 \times 8 \mathrm{~mm}$ with pixel resolution of approximately $6 \mu \mathrm{m}$. This pixel resolution is sufficiently small to resolve and track fine-soil particles of approximately 10$15 \mu \mathrm{m}$ (within the limits of the current application).

The maximum targeted flow velocity is approximately $100 \mathrm{~mm} / \mathrm{s}$, which at maximum zoom for the current optics will require a frame rate of $100 \mathrm{fps}$ for single exposure images. Highresolution cameras capable of collecting $100 \mathrm{fps}$ are prohibitively expensive for this study. An acceptable solution for this application is multiple exposure frames, which is achieved by multiple strobing of a single exposure, thus capturing the motion of fast-moving particles through the high-magnification image. A limit of the multi-exposure approach is that the seeding rate of particles must be relatively low to distinguish individual particle paths.

\section{LIGHTING}

To capture motion of fast-moving particles within a small field of view requires strobable, highintensity lighting. During the initial testing, side-, back-, and spot-lighting were evaluated with 
high-intensity LEDs. Side lighting with a diffuse backlight worked well with the clear glass beads, but low-angle, spot lighting was the most effective in illuminating fine particles transported through the media (both spherical and natural). A technical incompatibility between the spotlights and strobe controller was identified during the testing, which has been resolved by the manufacturer (and replacement components are currently being shipped to us). Images collected during initial testing were processed and analyzed to produce particle trajectories and speed. Image processing routines were developed to enhance detection and tracking of fine particles transported through the media. Following image processing, Particle Image Velocimetry (PIV) and Particle Tracking Velocimetry (PTV) are applied to the images.

One of the trial experiments performed during initial testing is discussed here as an example. The experiment included $1500 \mu \mathrm{m}$ soda-lime glass spheres as the filter media and a combination of $10 \mu \mathrm{m}$ Spherice ${ }^{\circledR}$ hollow microspheres $\left(\rho=1.10 \mathrm{~g} \cdot \mathrm{cm}^{-3}\right)$ and Vicksburg loess siltsized particles in suspension. The images captured detail the movement of sediment through the filter medium. Figure 16 depicts the motion of Vicksburg silt through the $1500 \mu \mathrm{m}$ spheres. Captured images were processed to increase contrast and fine-particle identification. The mean of all the images represents the background (the filter medium) whereas deviations from the mean represent fine particles moving with the flow. Using this concept, the image intensity of particulates in the flow is increased, improving identification. The subplots shown in Figure 16 represent a portion of the total field of view and a small subset of total video length. A silt particle is seen entering the field of view in subplot (a), highlighted with an arrow. The particle then moves over the following seven subplots through the porous medium. Due to the local geometry of the spheres, the particle encounters a complex flow field control by a combination of jets, sinks and recirculation regions with length scales on the order of the pore space. Subplots (b) and (c) show the particle moving towards the sink between two spheres that it passes between in subplot (d), exiting in a jet in subplot (e) before being drawn towards another sink in subplots ( $f$ ) and (g). Recirculation zones were also identified in the flow. These zones may be significant, as they act to reduce the true void fraction to some avaliable void fraction for net fluid motion.

Figure 17 presents the measured trajectory of multiple particles through the filter medium projected over the mean image. The particles move downward through the glass spheres with some trajectories overlaping revealing the turbulent characteristic of a chaotic flow pattern. Velocities of the sediment were calculated by dividing the change in $x$ and $y$ positions by the frame interval:

$$
u=\frac{\Delta x}{\Delta t} ; \quad v=\frac{\Delta y}{\Delta t}
$$


Calculated sediment velocities in this experiment ranged from $0.5 \mathrm{~mm} / \mathrm{s}$ to nearly $30 \mathrm{~mm} / \mathrm{s}$, demonstrating the complex nature of the hydrodynamics.

(a)

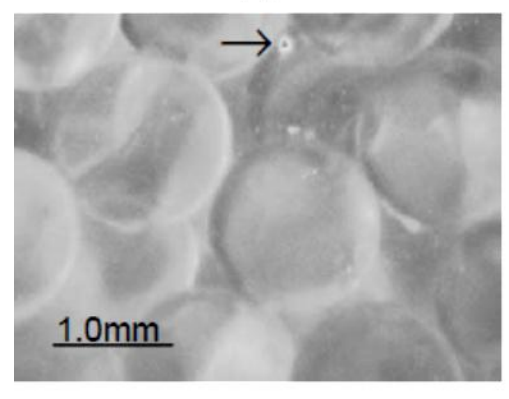

(b)

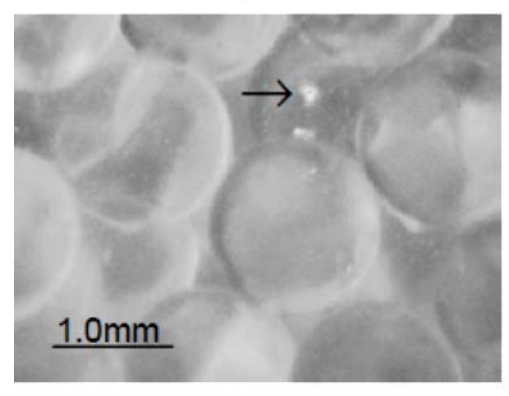

(c)

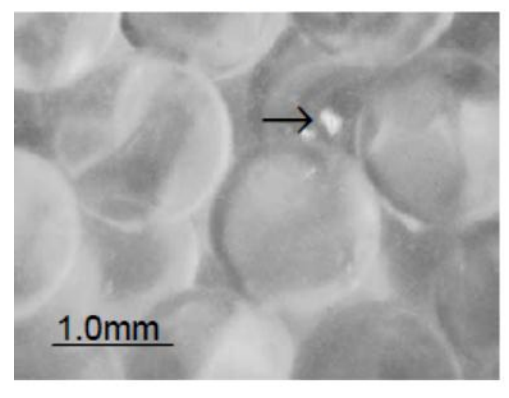

(d)

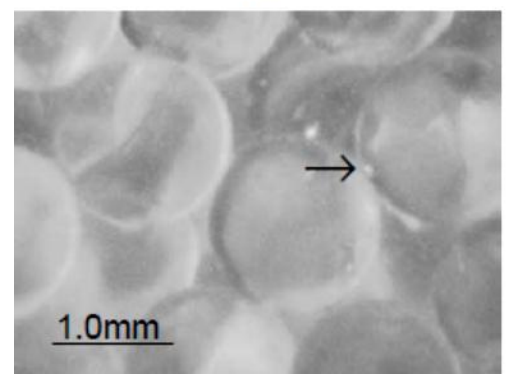

(e)

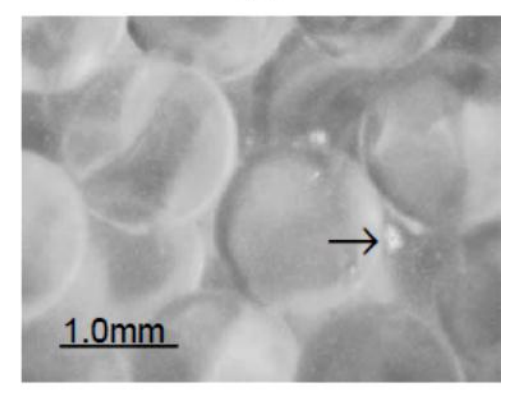

(f)

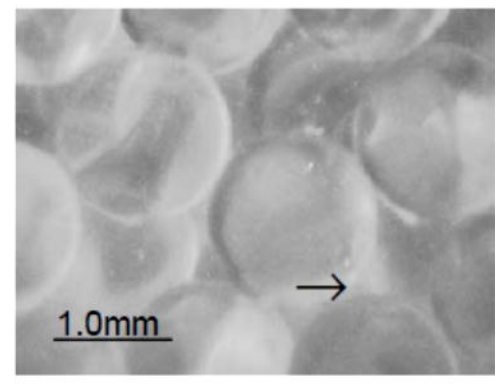

(g)

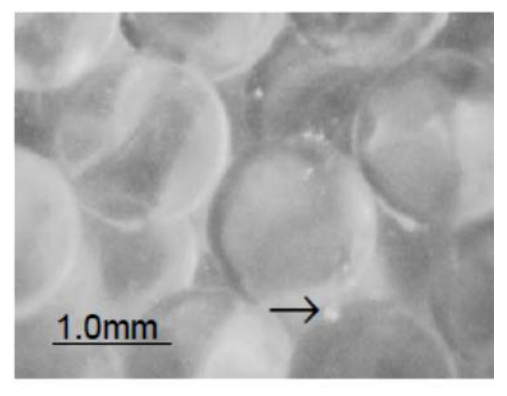

(h)

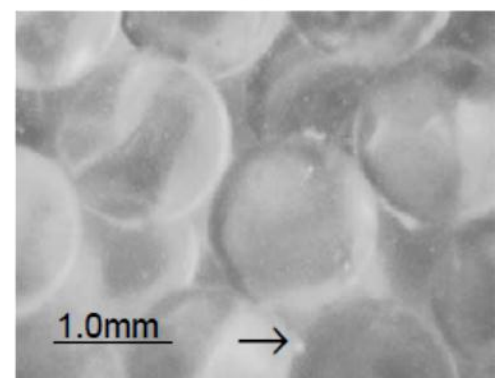

Figure 16. Transport of a silt particle through $1,500 \mu \mathrm{m}$, spherical filter media. Frame rate is $15 \mathrm{fps}$. 


\section{HEAD GRADIENT MEASUREMENT}

Pressure ports and a differential pressure gauge were installed as part of the initial testing task. The pressure ports are spaced $10 \mathrm{~cm}$ apart, centered vertically and laterally on the flow chamber side wall. The Omega Dyne differential pressure sensor measures the pressure difference between these two ports, which is logged by an Omega data logger. Pressure measurements will be converted to head gradient and mean velocity (Figure 18).

Filter media trapping efficiency will rely on physical sampling of the flow during experiments and of the filter media following experiments. Current plans are to collect all flow exiting the outflow tube and discrete samples from the inflow tube during experiments. Sample taps will be installed and testing of physical sampling will be performed in the coming weeks. Physical sampling of the filter media will be performed by either direct sampling of the drained media or freezing and sectioning of the media following experiments.

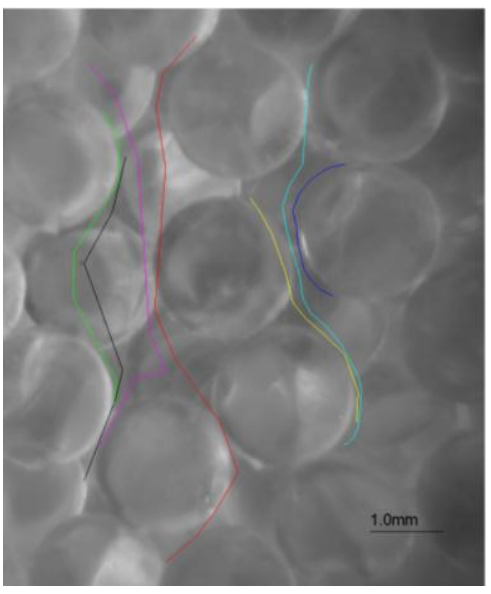

Figure 17. Particle trajectories for silt particles passing through 1,500 $\mu \mathrm{m}$, spherical filter medium. Individual trajectories are indicated by color.
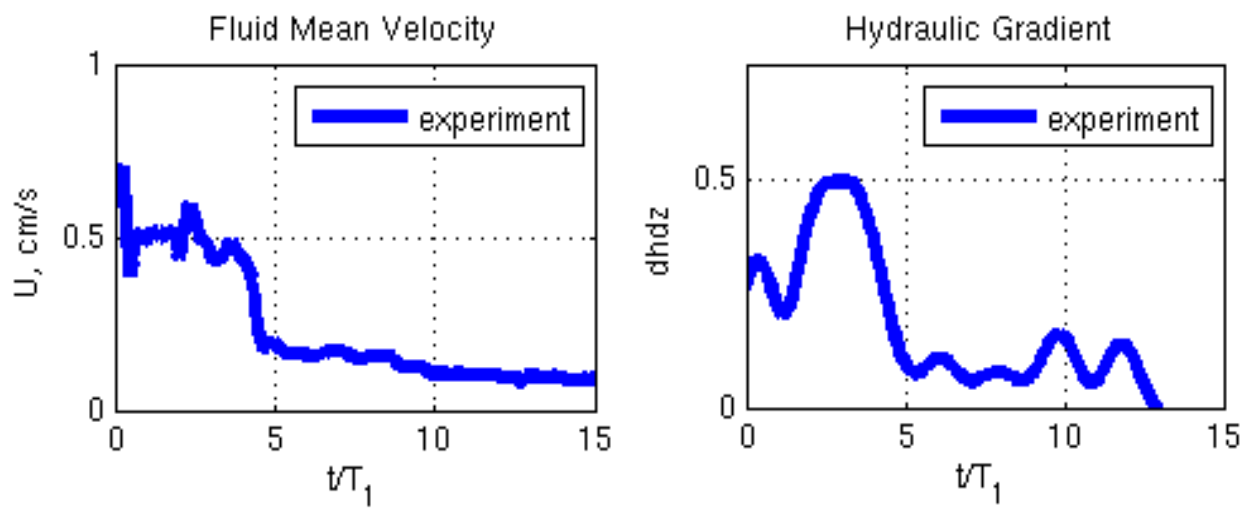

Figure 18. Experimental fluid mean velocity (derived from provided flow rates) and hydraulic gradients in the clean layer. 


\section{DAM SCALE MODEL}

\section{BACKGROUND}

In this section, we illustrate the numerical capabilities used to address different scenarios of the damage formation on the dam scale level. A typical transverse cross-section through an embankment (dam) is given in Figure 19.

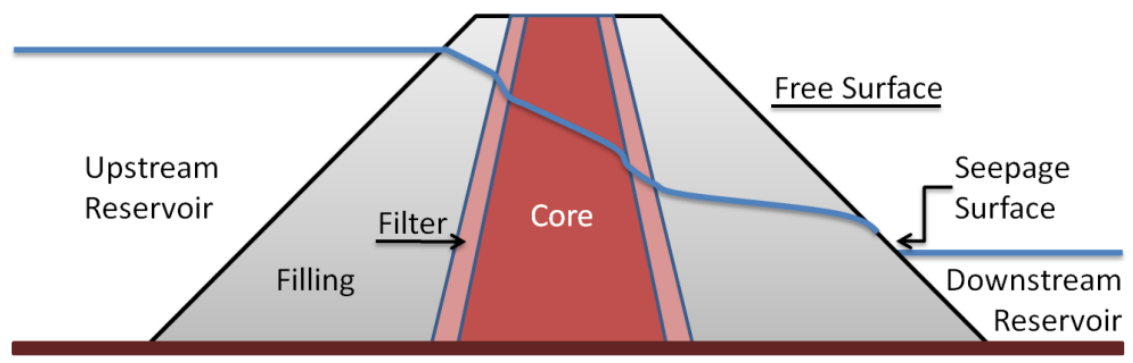

Figure 19. [Not to scale] Schematic of the physical domain for the computational analyses of dam stability under normal operational conditions and after dynamic loading. The blue line represents the water level upstream, through the dam, and downstream of the reservoir. The free surface is also known as phreatic surface.

\section{FLOW EQUATION}

The flow through porous media is governed by the conservation of mass (continuity) and the momentum (Darcy's) equations. When combined, we obtain the flow equation through a porous medium

$$
\operatorname{div}(K \operatorname{grad}(H))=S_{S} \partial_{t} H
$$

where $\mathrm{K}$ is the hydraulic conductivity tensor $[\mathrm{m} / \mathrm{s}], \mathrm{H}$ is the total head expressed as the sum of the pressure head $(P / \rho g)$ the potential head $(z)$ and the kinetic head $\left(V^{2} / 2 g\right)$ all expressed in [m]. The velocity $V$ is Darcy's. Because the kinetic head in porous media is relatively small, the total head is expressed as: $H=P / \rho g+z$, where $P$ is the pressure, $\rho$ is the fluid density, $g$ is the gravity acceleration $\left[\mathrm{m}^{2} / \mathrm{s}\right]$ and $z$ is the elevation $[\mathrm{m}]$ measured from a reference datum. The right hand of Equation (4 represents the change of mass (of water) with respect of time. $S_{S}$ is called the specific storativity of the porous media $\left[\mathrm{m}^{-1}\right]$ and represents the volume of water released from a volume of porous media under a unit head drop. It measures the capacity of the porous media to release water under the compressibility of water and the compressibility 
of the skeleton (grain). The specific storativity take the following form: $S_{s}=\rho g(\alpha+\beta \eta)$, where $\alpha$ is the grain compressibility, $\beta$ is the fluid compressibility and $\eta$ is the porosity of the medium (volume of void divided by the total volume). The hydraulic conductivity is defined as: $K=k \rho g / \mu$, where $k$ is the intrinsic permeability [ $\mathrm{m}^{2}$ ], and $\mu$ is the viscosity [Pa s]. It should be noted that the free surface represents a streamline and the position of the free surface is unknown a priori but has to be determined thus an additional condition on this surface is satisfied. At the seepage surface (Figure 19), being a streamline, imposes the condition $\partial H / \partial n=0$ to be satisfied along the seepage surface. Here $n$ represents the outward vector normal to the slope downstream of the dam. In addition, however, the pressure must be zero on the surface as this is exposed to atmosphere, thus we must have $H=z$.
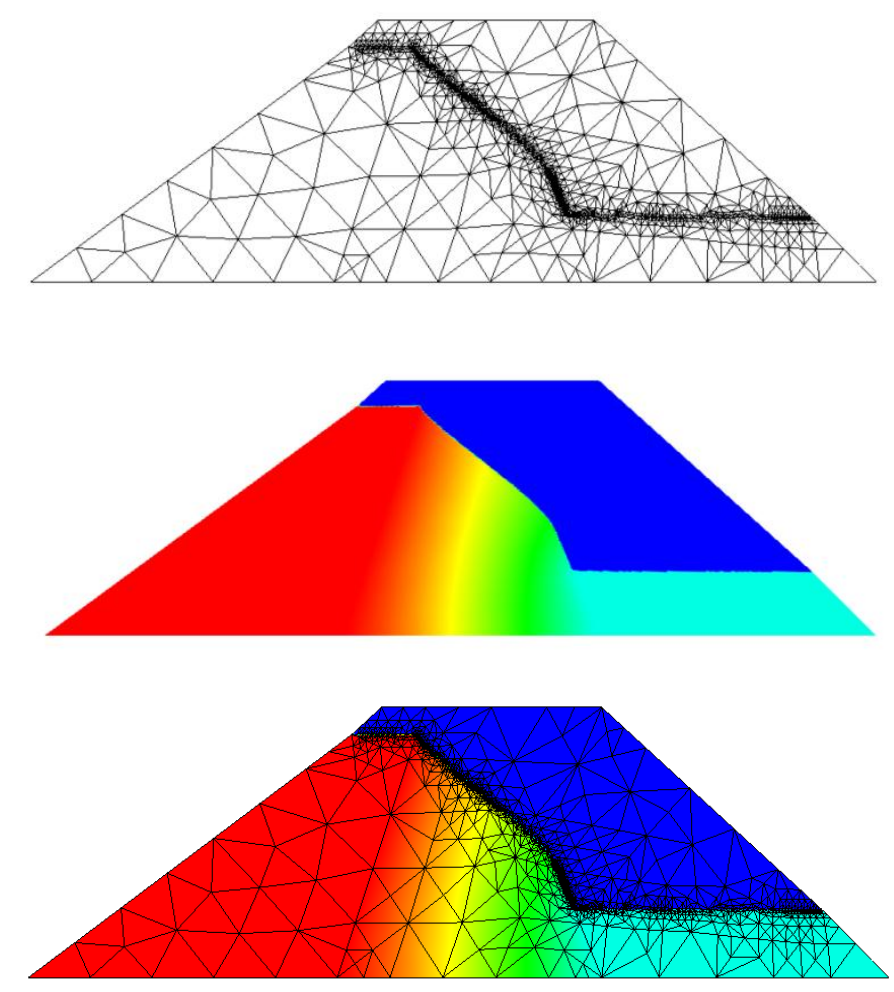

Figure 20. Example of FEM-AMR solution to pressure distribution within an embankment. The embankment is composed of a single core with a very low hydraulic conductivity. Upstream and downstream of the core are relatively high filling. Top: FEM-AMR mesh capturing the free surface.

Middle: head (pressure) distribution along the embankment. Bottom: composed solution.

This problem is non-linear in nature and the numerical solution maybe approached iteratively. Starting with a prescribed free surface streamline the standard problem is solved. A check is carried out to see whether $H=z$ is satisfied and, if not, an adjustment of the surface is carried out to make a new $z$ equal to $H$ that was just found. It is worth noting that the free surface is also defined by the boundary between the saturated and unsaturated zone (Figure 20). A few 
iterations of this kind show that the convergence is reasonably rapid. Alternative methods, including special variations of principles for dealing with this problem, have been devised over the years; others choose a third type-like penalty boundary condition to resolve for the seepage surface. Moreover, the free surface is usually a non-linear function, continuous but not differentiable when encountering domain with different geotechnical properties (e.g., hydraulic conductivity). In order to capture the free surface, one may use the finite element method with tetrahedral elements. Furthermore, element sizes may need to be adapted (refined) to resolve the interface if needed. To accomplish a fine resolution and adaptive mesh refinement scheme on the finite element unstructured grid is adopted.

\section{TRANSPORT OF FINES EQUATIONS (TYPE I)}

Transport of fines in porous media has received a considerable attention the last three decades. For completeness, hereafter we abbreviated the most commonly used model. The advective dispersive equation of a 'tracer' of fine is given by:

$$
\operatorname{div}(D \operatorname{grad}(C)-U C)=R \partial_{t} C
$$

where $D$ is the dispersion coefficient, $C$ is the concentration of fines, $U$ is the advective velocity (=Darcy's divided by the porosity, $\eta$ ), $R$ is the retardation coefficient $R=\left(1+\rho_{b} k_{d} / \eta\right.$ ), where $\rho_{b}$ is the bulk density and $k_{d}$ is a partition coefficient. We begin by introducing a model of fine transport with retention kinetics as discussed in de Marsily (1986). Let $\eta_{0}$ be the initial porosity, porosity when fines are not present, of a one-dimensional porous domain of cross sectional area $A$.

Because transported particles are assumed to have a volume, we measure the concentration of $C(x, t)$ of transported fine in volume of fine per unit volume of liquid-fine suspension. We assume that the density of the fines is the same as that of the carrying liquid. We denote by $\sigma=\sigma(x, t)$ the volume of retained, or immobile, fines per unit volume of porous medium. Let $V$ be the Darcy velocity of the fine-carrying fluid, which the volume of suspension per unit area per unit time, and $\mathrm{J}=\mathrm{J}(\mathrm{x}, \mathrm{t})$ denotes the volumetric flux of fines, measured in volume of fines per unit area per unit time. Here Darcy's velocity is not constant, because if the medium becomes clogged, the flow may terminate. If $\eta=\eta(x, t)$ is the changing porosity of the medium, then we may write the volume balance equation in an arbitrary section $[a, b]$ of the medium as:

$$
\frac{d}{d t} \int_{a}^{b} C \eta A d x=A\left(J(a, t)-J(b, t)-\int_{a}^{b} \frac{d \sigma}{d t} A d x\right.
$$


Which simply states that the rate of change of the total volume of fines in the section must equate the rate that they flow in, minus the rate they flow out, minus the rate of fines is accreted (adsorbed, attached ...) onto the solid matrix. Similarly we obtain the volume balance equation in local form as:

$$
\frac{\partial(C \eta+\sigma)}{\partial t}=-\frac{\partial J}{\partial x}
$$

The first constitutive equation is the form of the porosity-accretion relationship. We assume for the sake of simplicity that:

$$
\eta=\eta_{0}-\beta \sigma
$$

where $\beta$ is a non-negative constant called the compaction (or filling) factor. Thus the volume of retained fines occupies a volume $\beta \sigma$ of the pore space. Next, we assume that the rate of retention is proportional to the concentration of fines in solution and to the velocity of the flow, and it depends, perhaps, nonlinearly on the volume concentration of fines already retained (accreted); mathematically speaking, this leads to:

$$
\frac{\partial \sigma}{\partial t}=\lambda U C F\left(\frac{\sigma}{\eta_{0}}\right)
$$

where the positive constant $\lambda$ is called the filtration (filter) coefficient, and $F$ is a non-negative non-increasing function. In this model detachment is negligible, but can be added. We further assume that the volumetric flux $\mathrm{C}$ contains an advective term and a dispersion term, such that: $J=U C-\eta D \partial_{x} C$. Combining the above equations give the model for fines filtration:

$$
\begin{gathered}
\frac{\partial(C \eta+\sigma)}{\partial t}=-\frac{\partial}{\partial x}\left(\eta D \frac{\partial C}{\partial x}-U C\right)+S \\
\eta=\eta_{0}-\beta \sigma \\
\frac{\partial \sigma}{\partial t}=\lambda U C F\left(\frac{\sigma}{\eta_{0}}\right)
\end{gathered}
$$


where $S$ is the source term that describes erosion of soil particles. The filter coefficient, $\lambda$, which has dimension of $\left[\mathrm{s}^{-1}\right]$, could depend in a complicated manner on the diameters of the grains and particles, the viscosity of the fluid, the porosity itself, and other quantities affecting the efficiency of the "fabric" as a filter.

Generally, one would expect a strong dependence on the porosity. Hydrogeologists have fit various functional forms of $\lambda$ to collected data and the reader can consult Herzig et al. (1970), Rajagopalan and Tien (1976), or de Marsily (1986) for references to experimental work. For the current analysis we will use a variable rate law for the accretion function $F$ assuming a kinetic factor of the form: $F\left(\sigma / \eta_{0}\right)=1-\beta \sigma$. Several theoretical and experimental relationships are available in the literature. Common kinetic functions are: $F\left(\sigma / \eta_{0}\right)=1-\beta^{2} \sigma^{2}$, $F\left(\sigma / \eta_{0}\right)=(1-\beta \sigma)^{\alpha}, \alpha=1 / 2$ or $3 / 2$. These particular relationships are also useful when seeking analytical solutions.

It is worth noting that, so far, the model ignores the permeability changes of the porous medium. Permeability is closely correlated to the porosity, (e.g. Kozeny-Carman where $k / k_{0} \sim \eta^{3} /(1+\eta)^{2}, k_{0}$ is the initial permeability and $\eta$ is the porosity).

Once the fines are accreted, the porosity changes, so does the permeability, and it will take a greater and greater pressure gradient over the length of the domain to keep the velocity constant or given a constant gradient, the velocity keep decaying with time till flow ceases. One would not expect the model to be valid in situations near total clogging. In a more accurate model, Darcy's law comes into play, and the above system of equations needs to be solved simultaneously with the flow equation introduced in the previous subsection, which is the approach used here.

\section{DAM SCALE SIMULATIONS}

\section{NORMAL OPERATIONAL CONDITIONS}

Simulation of the embankment under normal operational conditions (no dynamic loading) has been conducted. Figure 21 shows the Finite Element mesh with refinement around the free surface to capture its shape. Upstream and downstream boundary conditions are clamped pressure conditions. Using the iterative scheme presented in the previous subsection, one can start with a straight line connecting both pressure boundary conditions with saturated permeability below and unsaturated permeability above the initial guess of the free surface. After a few refinement stages (see Figure 21), the flow equation converges to the "steady" solution. 

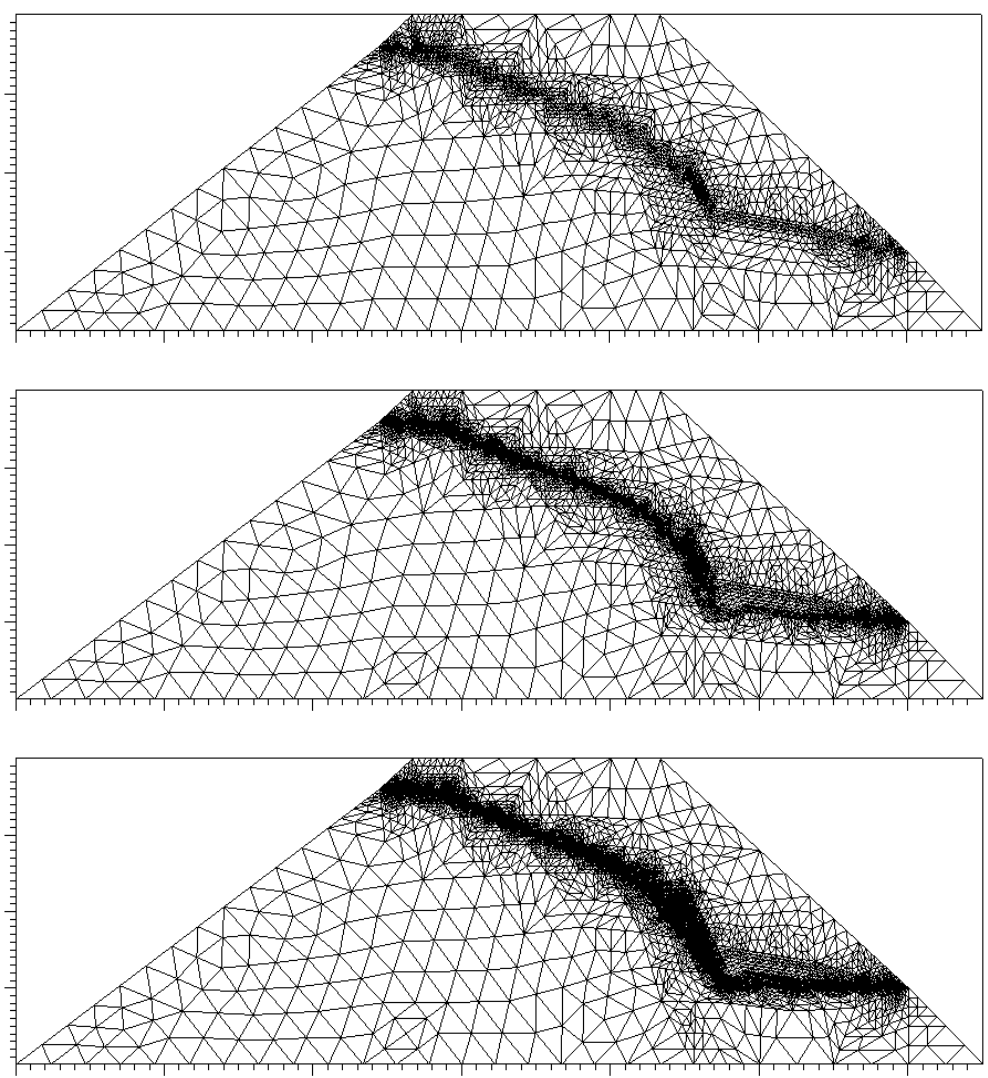

Figure 21. Example of FEM-AMR around the free surface. At each stage the number of nodes, and thus element, increases around the free surface to capture the solution. The number of nodes are $\sim 2,000$, $\sim 14,300$, and $\sim 44,750$ for stages 1,2 , and 3, respectively (from top to bottom).

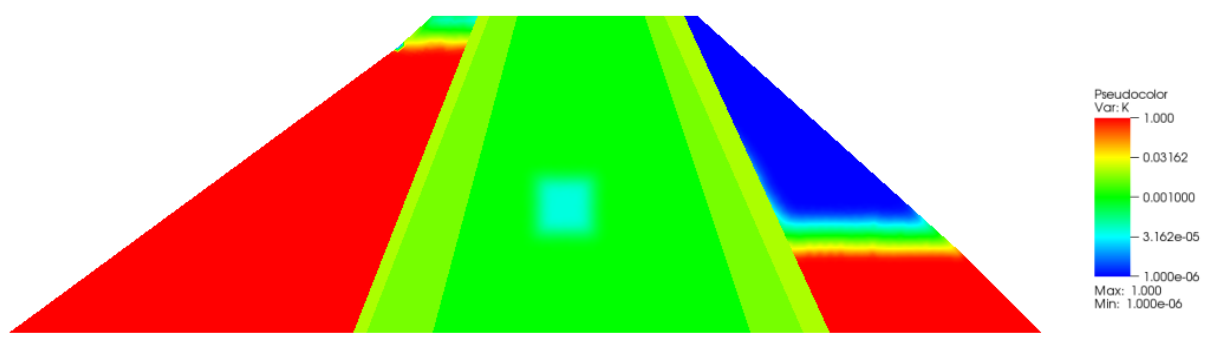

Figure 22. Hydraulic conductivity, $\mathrm{K}$, within the embankment. The distribution of the permeability reflects the 'design' of the embankment. The difference in the hydraulic conductivity between the filling and the core is about 6 orders of magnitude.

The hydraulic conductivity changes as function of the 'density' (concentration) of fine. The higher the density of fine is the lower the hydraulic conductivity. The function form used to accomplish this coupling between the hydraulic conductivity and the concentration of fine is simple and takes the following form: $K=K_{0}(1-\zeta \mathrm{C})$, where $K_{0}$ is the initial distribution of the hydraulic conductivity (e.g. Figure 22 ), $\mathrm{C}$ is the concentration of fines and $\zeta$ is a positive constant, close to unity. This equation reads that when no fines are present, the conductivity is 
unchanged; otherwise $K_{0}$ is reduced by a factor, $\zeta C$. For demonstration purposes, the concentration of fine is limited between 0 (no fines) and 1 (dominant fines).

Figure 23 and Figure 24 depict the time evolution of mobilized-fines migration (without retention) within the embankment. Furthermore, Figure 23 and Figure 24 show the hydraulic conductivity contrast within the embankment (opaque regions). Time snapshots are from left to right and top to bottom. This simulation represents a scenario where there is a small amount of mobilized fines that do not necessarily trigger the physical filtration of the fines and thus the alteration of the hydraulic conductivity and the possible creation of 'wormhole' flow regions. However, it should be noted that the initial condition here for the hydraulic conductivity field is set at an even lower permeability than initial core permeability. Despite that this scenario is unrealistic for the problem at hand, it is of great importance, because most published results (especially of tracer transport in porous media) deal with similar conditions (de Marsily, 1986) and do not necessarily deal with dynamic loading (see next subsection).

Not surprisingly, the flow streamlines, upstream of the source of fines, diverge away from the fine source, then converge downstream of the fine source to reach the downstream embankment boundary, which leads to an increase of velocity at the upper and lower edge of the fine-source, resulting in a downstream deformation of the source followed by an ovalization of the source itself. Fines continue to migrate and disperse downstream within the core until they encounter the filter region where the flow is relatively higher, forcing the fines to migrate downward toward the higher hydraulic conductivity filling (shell in Figure 22). It is a matter of time until the fines find their way out downstream of the embankment, because no retention is allowed.

\section{DYNAMIC LOADING WITHOUT FILTER ZONE}

To alleviate the constraint on the initial conditions previously presented, we simulated a local increase of the hydraulic conductivity at the source of mobilized fines which mimics a dislocation region. To illustrate the difference between the current analysis and no-increase of hydraulic conductivity scenario, we depicted in Figure 25 the head (pressure) distribution within the core. Furthermore, Figure 25 (bottom) depicts the streamlines emanating from the upstream boundary. As expected, the flow streamlines converge toward the high hydraulic conductivity region, then diverge again downstream of the region and make their way to the left boundary.

Temporal evolution of the fines is depicted in Figure 26. Contrary to what has been observed in the previous simulation, the fine-source gets stretched vertically and squeezed horizontally downstream due to the high upstream velocity and the divergence of the streamlines downstream of the source of fines. It should be noted here that the fines were not allowed to 
disperse, but mainly to advect (higher Peclét number). Similarly to the previous simulation, fines are not allowed to attach, and physical filtration does not take place.
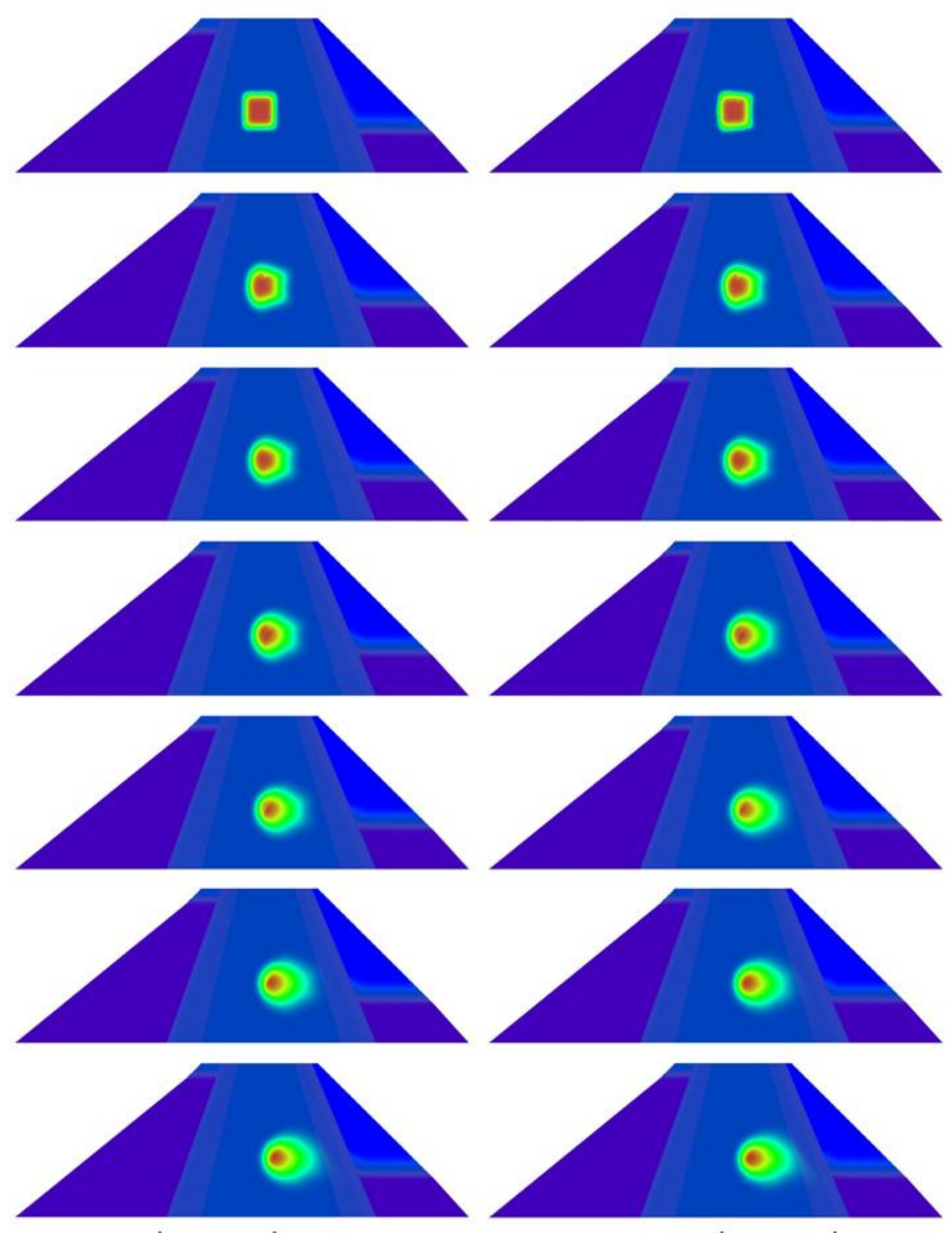

Figure 23. Time-evolution (snapshots) of fine migration within the embankment (continued). Time flows from left to right and top to bottom. 

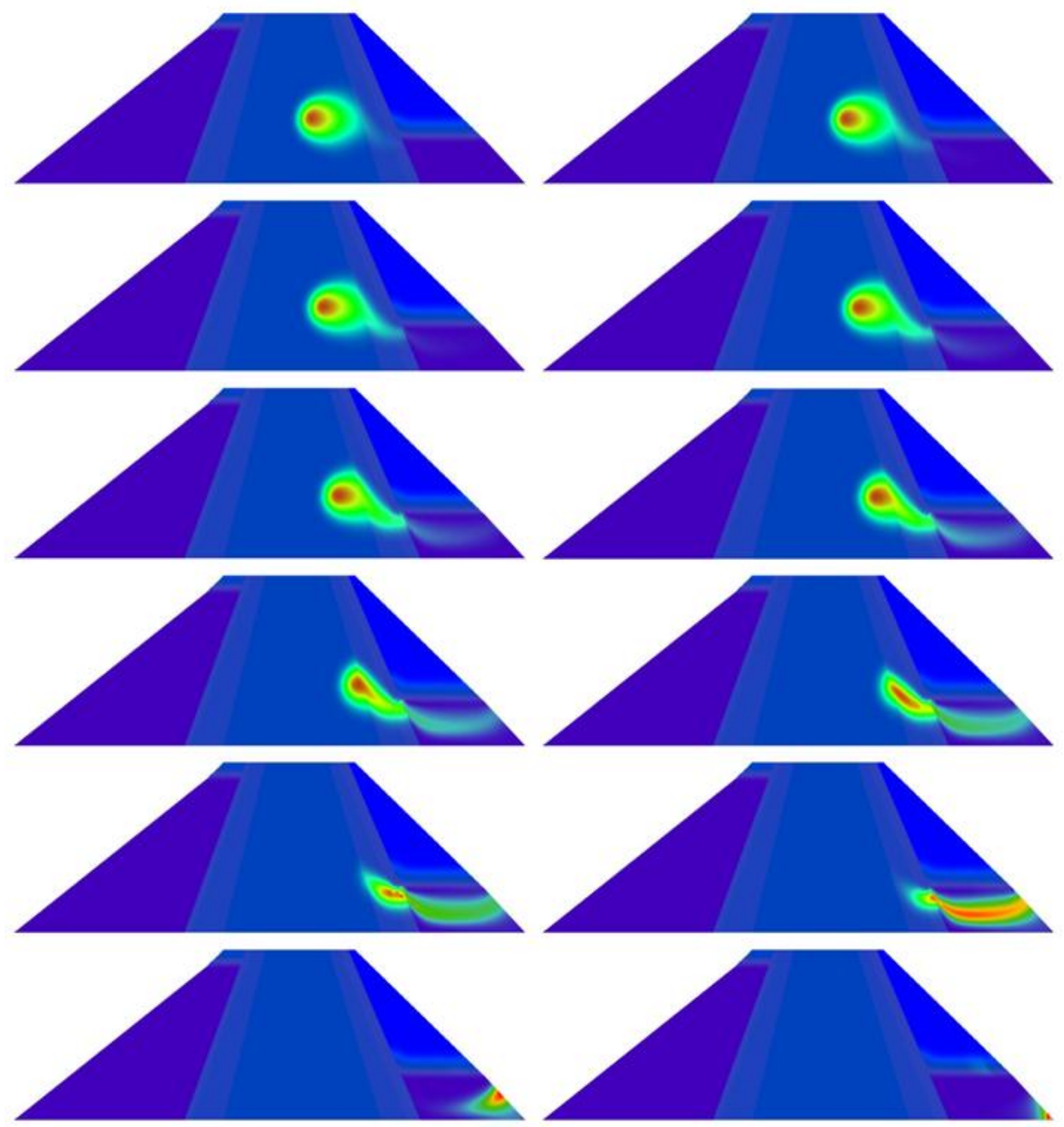

Figure 24. (Continued) Time-evolution (snapshots) of fine migration within the embankment. Time flows from left to right and top to bottom. Simulations shown here do not include attachment of the fine particles; once mobilized they are washed out.

\section{DYNAMIC LOADING WITH FILTER ZONE}

In this subsection we analyze the effect of entrapment (attachment or physical filtration) on the distribution of mobilized fines within the embankment. Because the kinetic of entrapment is essential to the fate of fines, we explored the effect of kinetic term in equation (10). We shall 
call it here: attachment isotherm. A linear and non-linear case were simulated: $F\left(\sigma / \eta_{0}\right)=1-\beta \sigma$ and $F\left(\sigma / \eta_{0}\right)=1-\beta^{2} \sigma^{2}$. Simulations conducted here are based on the previous analysis, except that the fines are allowed to filtrate, washout, and impact the hydraulic conductivity within the embankment.

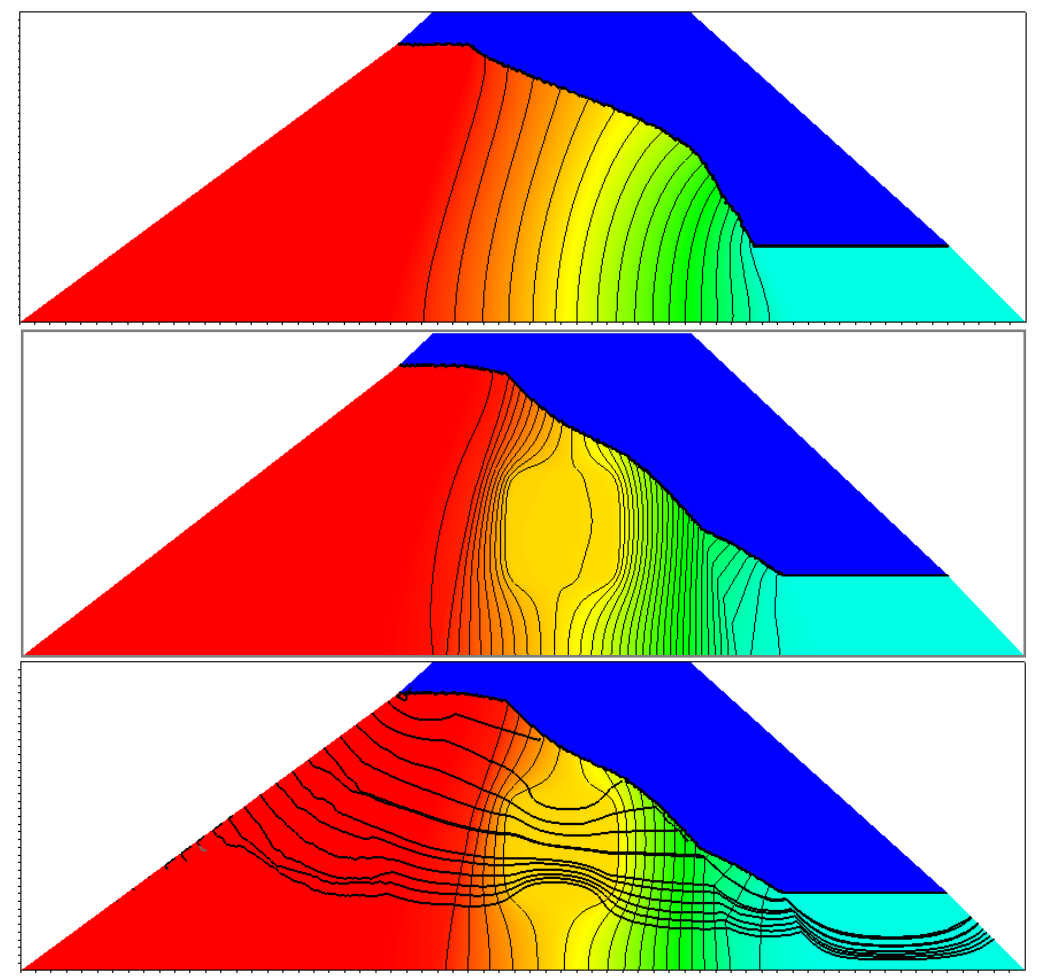

Figure 25. Head distribution under normal condition of embankment (top) and under dynamic load (middle). Head distribution and streamlines under dynamic load (bottom). Streamlines show convergence of the flow toward the high conductivity area. High conductivity area is due to dislocation of the embankment from the core of the dam. The creation of high conductivity zone impacts the head distribution and the shape of the free surface.

Figure 26 and Figure 27 depict snapshots in time of attachment and washout of fines, respectively, for the linear isotherm, while Figure 28 and Figure 29 depict the same processes for the non-linear kinetic isotherm. It is worth noting that time flows from left to right and top to bottom. Interestingly, in both cases, one can observe the growth of the attached fine zone. At each instance, the fines are allowed to attach each time they encounter a new core zone immediately at the zone interface, thus building a layer (film) of jammed, attached fines. This process is called physical filtration. The film of deposit fines will subsequently impact the hydraulic conductivity, and thus, the flow through the embankment. The area affected by the fine filtration is considerably different from one kinetic isotherm to another. Indeed, the nonlinear isotherm induces a larger zone of fine deposits, and thus, larger zone of reduced hydraulic conductivity. 

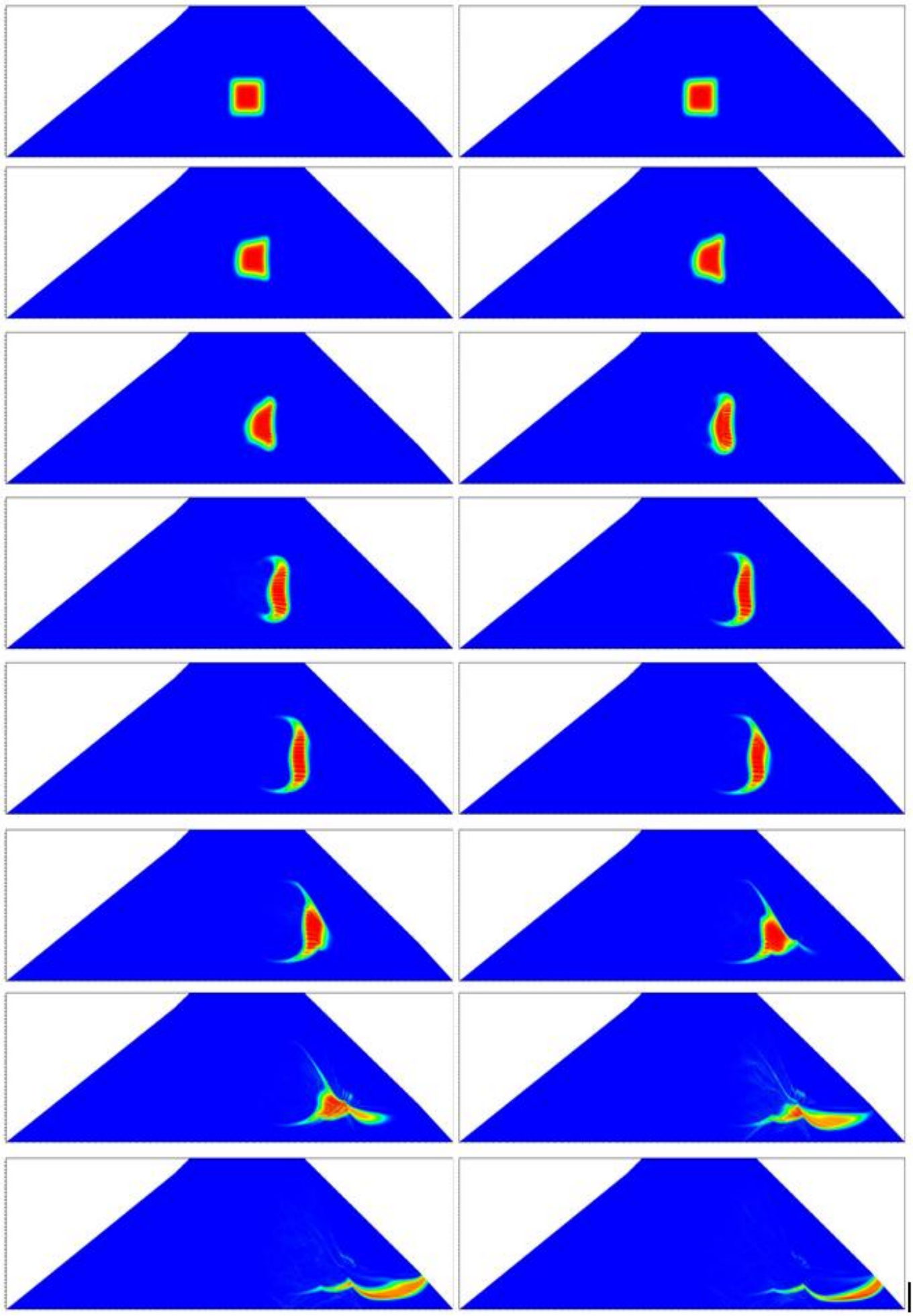

Figure 26. Time-evolution (snapshots) of fine migration within the embankment. Time flows from left to right and top to bottom. Simulations shown here do not include attachment of the fine particles; once mobilized they are washed out. However, the mobilization of fine particles leads to a high conductivity zone: zone of dislocation of embankment from the core of the dam. 
It was estimated that $50 \%$ of the total fine mobilized were attached and retained within the embankment in the non-linear case, compared to only $30 \%$ in the linear case. In reality, cores of dams are made of very fine soils, mainly clays. Clay minerals are electrically charged. Clays have a high cation-exchange capacity, which is defined as the degree to which a soil can absorb and exchange cations and render the attachment process (filtration) a non-linear function of the density (concentration) of fines. It is well documented (de Marsily 1986) that the linear kinetic isotherm for filtration model does not predict well experimental data. Needless to say, more parametric studies need to be conducted on the effect of the kinetic isotherm parameters $\beta$, and $\alpha$, which are determined for specific soil type and physical conditions. In addition, these two parameters can be obtained from comparisons with the mesoscale numerical simulations and experiments.

\section{DUAL POPULATION CONTINUUM MODEL FOR TRANSPORT OF FINES (TYPE II)}

TRANSPORT OF FINES

For saturated porous media, the transport equation may be used to describe the advection, dispersion, and deposition of fines

$$
\frac{\partial R N}{\partial t}=\operatorname{div}\left(D \operatorname{grad}(N)-V_{P} N\right)-\frac{f}{\pi R_{P}^{2}} \frac{\partial O}{\partial t}
$$

where $N$ and $O$ are dependent variables corresponding to the number concentration of suspended particles and the local fraction of the immobile matrix that is covered by deposited particles, respectively. Model parameters contained in the transport equation include the interstitial particle velocity $V p$, the hydrodynamic dispersion coefficient $D$, the particle radius $R p$, and the specific surface area $f$. The specific surface area for uniform, spherical collector grains may be determined as a function of grain size and bed porosity from an analytical expression.

\section{ADVECTION OF FINES}

Particles suspended within an aqueous porous medium will generally travel at slightly higher velocities than the advecting fluid medium. This enhancement of particle velocity with respect to fluid velocity is a result of a particle's finite dimensions, which prohibits it from contacting the slower moving fluid near the pore boundaries. 

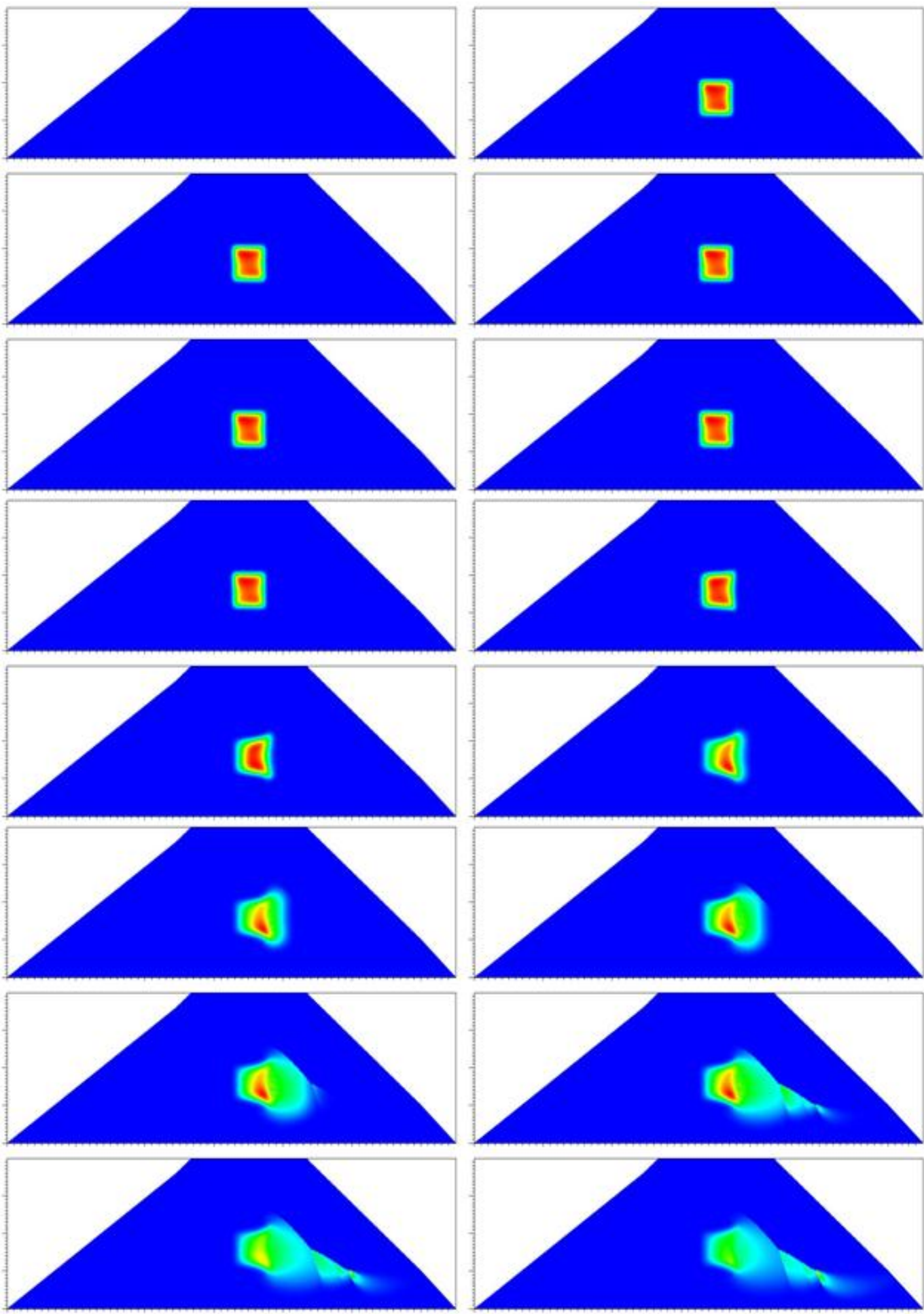

Figure 27. Time-evolution (snapshots) of fine entrapment (filtrations) within the embankment. Time flows from left to right and top to bottom. Simulations shown here do include attachment of the fine particles; once mobilized they are either attached or washed out. Attachment "isotherm" is $\mathrm{F}\left(\sigma / \eta_{0}\right)=1-\beta \sigma$. 

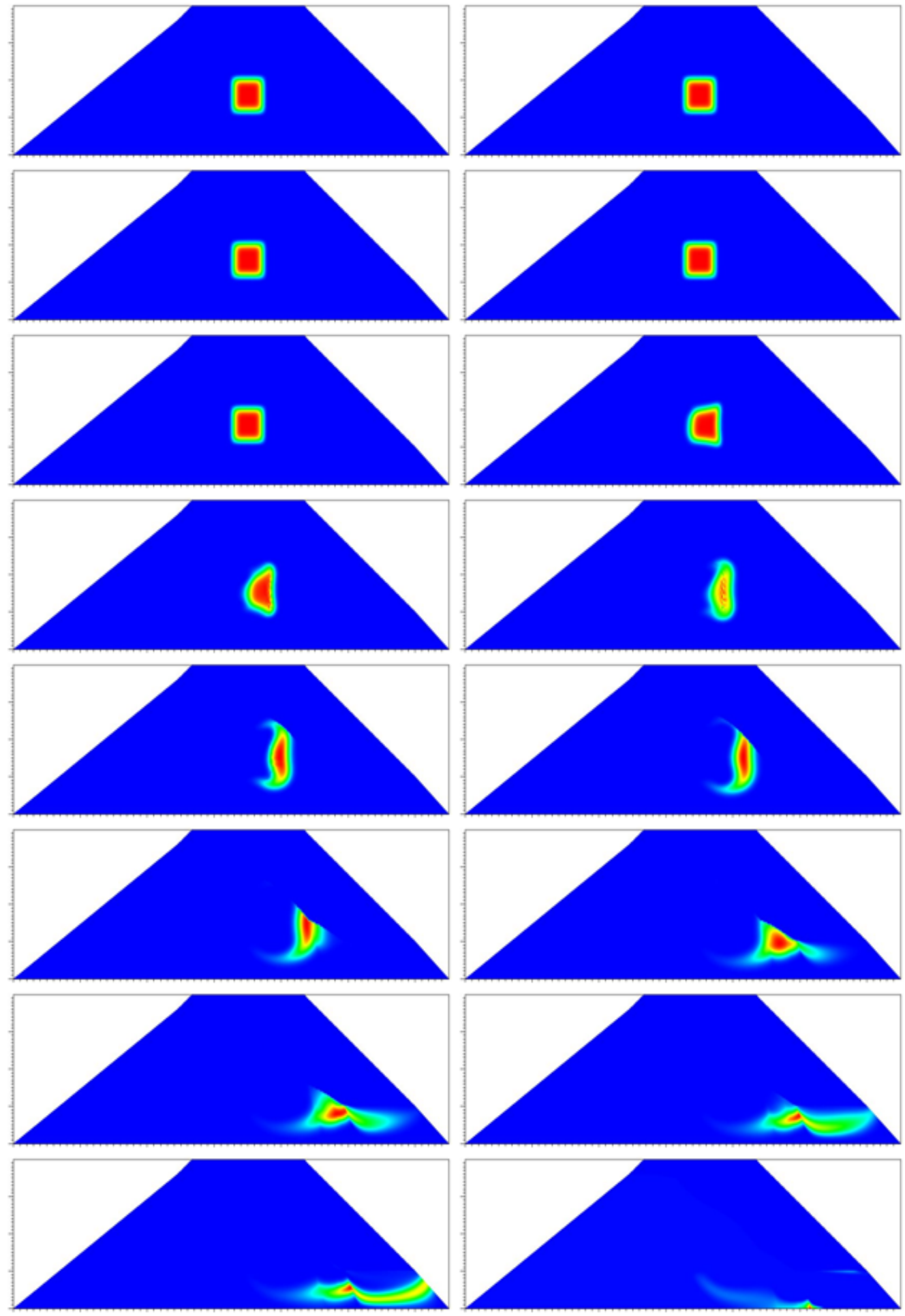

Figure 28. Time-evolution (snapshots) of fine migration (washed) within the embankment. Time flows from left to right and top to bottom. Simulations shown here do include attachment of the fine particles; once mobilized they are either attached or washed out. Attachment "isotherm" is $\mathrm{F}\left(\sigma / \eta_{0}\right)=1-\beta \sigma$. 

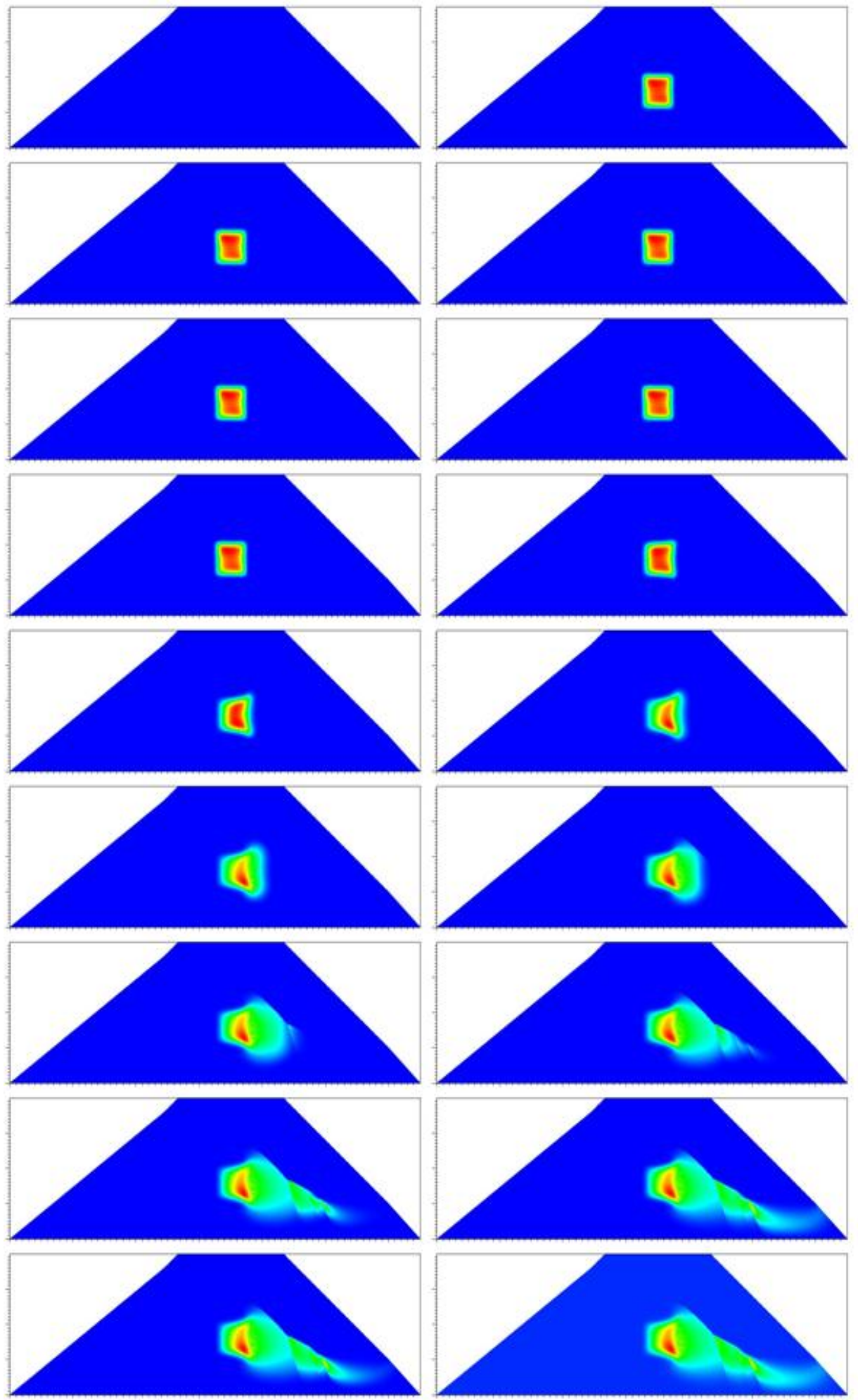

Figure 29. Time-evolution (snapshots) of fine Entrapment (filtration) within the embankment. Time flows from left to right and top to bottom. Simulations shown here do include attachment of the fine particles; once mobilized they are either attached or washed out. Attachment "isotherm" is

$$
F\left(\sigma / \eta_{0}\right)=1-\beta^{2} \sigma^{2} \text {. }
$$



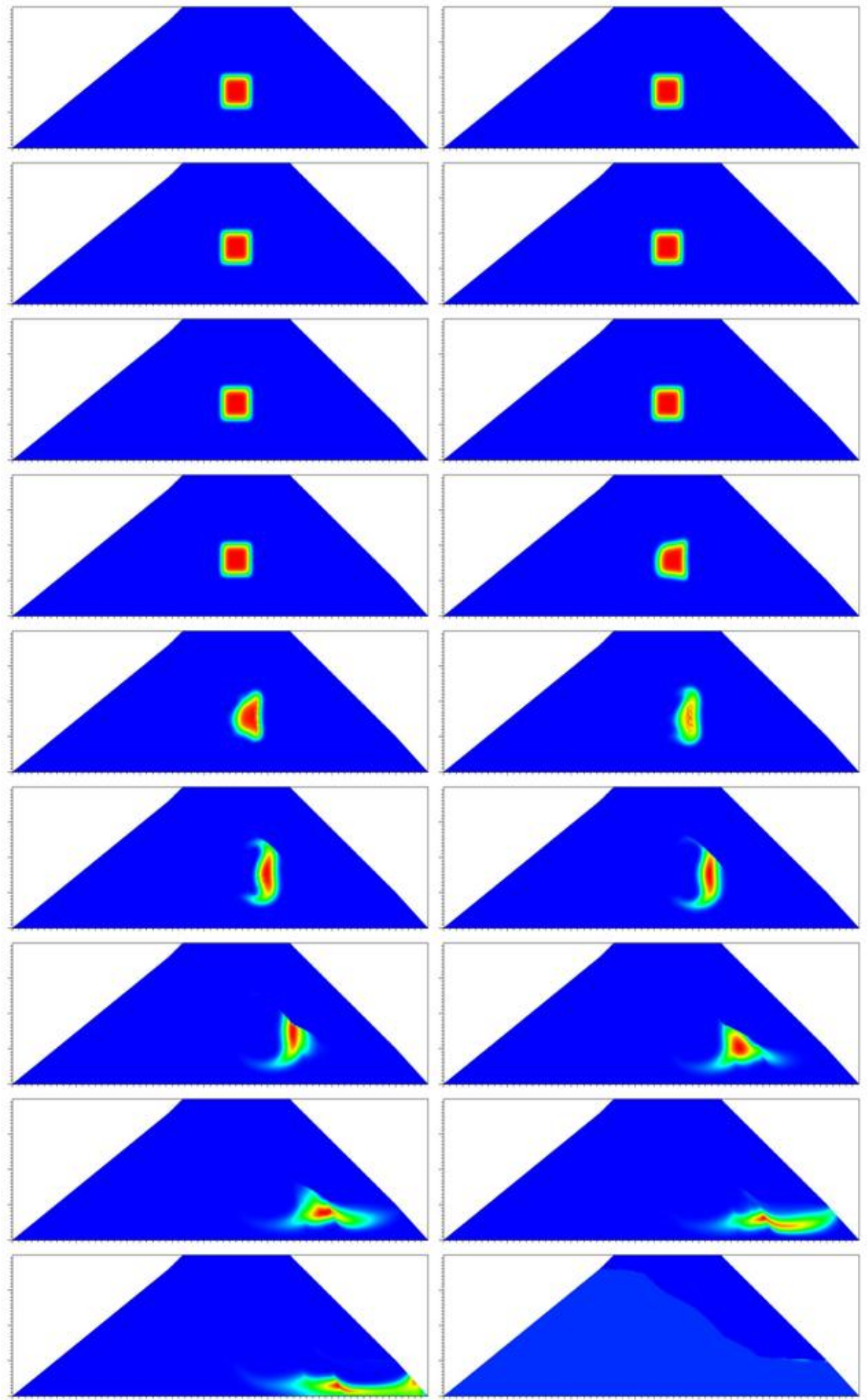

Figure 30. Time-evolution (snapshots) of fine migration (washed) within the embankment. Time flows from left to right and top to bottom. Simulations shown here do include attachment of the fine particles; once mobilized they are either attached or washed out. Attachment "isotherm" is

$$
F\left(\sigma / \eta_{0}\right)=1-\beta^{2} \sigma^{2} \text {. }
$$


An expression that relates the velocity of a suspended particle to the interstitial fluid velocity and aspect ratio between fine radius and pore radius, adapted for spherical particles flowing in a cylindrical tube, is given by

$$
V_{P}=\frac{U}{\eta}\left[2-\left(1-\frac{R_{P}}{\mathfrak{R}}\right)^{2}\right]
$$

where $U$ is the superficial fluid (Darcy) velocity, $\eta$ is the porosity, and $\mathfrak{R}$ is the pore radius. In natural soils, in particular clays and loams develop surface charges when saturated. Grains surfaces contain "chemical" patch charge heterogeneities. A two patch charge heterogeneity model called mobile-immobile fraction can be used to mimic the chemical heterogeneity. Thus $O$ becomes a linear combination of both fractions: $O=\varepsilon O_{f}+(1-\varepsilon) O_{u}$. The heterogeneous parameter $\varepsilon$ is the fraction of spatially available surface area that is favorable for the fines deposition.

\section{KINETICS AND DYNAMICS OF FINE DEPOSITION}

Most cases involving deposition of fines, the rate of deposition declines as retained particles block subsequent attachment. Because of blocking effects, the kinetic rate equation must be modified to consider the dynamic or transient aspects of fines deposition. Deposition dynamics that are produced by blocking effects may be quantified by appending a dynamic blocking function to the kinetic rate equation. The dynamic blocking function $B(O)$ describes the probability of a particle contacting a portion of collector surface that is unoccupied by previously deposited particles. It provides a correction for the blocking effects of deposited particles on the initial deposition rate (this is similar to $F(\sigma)$ ). Deposition dynamics produced by blocking effect may be quantified by:

$$
\frac{\partial O}{\partial t}=\pi R_{P}^{2} k \eta B(O)
$$

where $k$ is the kinetic rate constant also known as the particle transfer function, and $B(O)$ is the blocking function. The simplest form is $B(O)=1-\beta O, \beta$ is the excluded area parameter. It is worth noting that this functional form is exactly the same as in Type I model. Several other blocking non-linear functions are given in the literature - the common one is the so-called random sequential deposition (RSD). Similarly the kinetics can be split into mobile and immobile phases: 


$$
\frac{\partial O}{\partial t}=\pi R_{P}^{2} \eta\left[\varepsilon k_{f} B\left(O_{f}\right)+(1-\epsilon) k_{u} B\left(O_{u}\right)\right]
$$

The dynamic rate equations for geochemically heterogeneous surfaces along with the transport equation comprise the governing equations of the transport model for fines in geochemically heterogeneous porous media. Because the kinetic of entrapment is essential to the fate of fines we explored a linear kinetic case: $F\left(\sigma / \eta_{0}\right)=1-\beta \sigma$ for Type I and $B(O)=1-\beta O$ for Type II models.

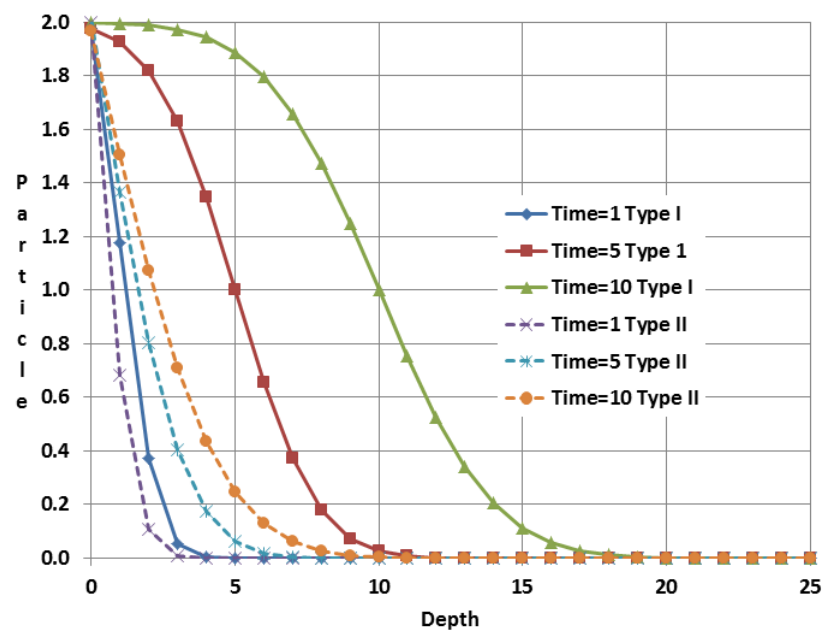

Figure 31. Front migration as function of depth and different times for Type I and Type II fine migration models.

A simple comparison between Type I and Type II of the propagation of the front is given on Figure 31 for the same parameters. As one can see that the Type II model front comes to a halt long before Type I model. We would not be surprised that using group theory (similarities) one can mathematically reduce, for a subset of common parameters, the Type II model into Type I model. Simulations conducted here warrant the fines to filtrate, washout and impact the hydraulic conductivity within the embankment. Snapshots in time of attachment and washout of fines, respectively, for the linear isotherm as similar to those depicted in Figure 27 and Figure 28 and are not re-produced here.

\section{SIMULATION OF EMBANKMENT AFTER DYNAMIC LOADING WITH ATTACHMENT TYPE II}

We have re-simulated the same problem in the previous section except we are using Type II attachment model, the kinetics were still linear (Langmuirian). It was estimated that $80 \%$ of the total fine mobilized were attached and retained within the embankment in the Type II case compared to only $30 \%$ in the Type I model both using linear kinetics of attachments. As stated 
before, in real life, core of dams are made of very fine soils, mainly clays. Clay minerals are electrically charged and have a high cation-exchange capacity and render the attachment process (filtration) a non-linear one function of the density (concentration) of fines. It is expected that non-linear kinetic isotherm for filtration model does mimic well the real physical processes of filtration. We believe that using non-linear fines attachment model will lead to a higher retention rates of fines thus a more certain self-rehabilitation of the embankment.

In this study we have implemented a non-linear filtration model to simulate the impact of mobilized fines on the fate of flow and stability of embankments. The model solves simultaneously, the flow, attachment and washout of fine particles within a heterogeneous porous media. The model uses state of the art finite element method with adaptive mesh refinement that not only captures the interface between water dense with fines/clear water but also the non-linearity of the free surface itself. Through this preliminary analysis, one concludes that the attachment kinetic isotherm is the key function of the model and more parametric studies need to be conducted to assess the sensitivity of the kinetic isotherm parameters on the stability of the embankment. It is worth mentioning that the model, for the more realistic, non-linear kinetic isotherms has predicted a self- rehabilitation of the breached core with retention of $80 \%$ of the mobilized fines. As stated in the analysis Type I, a more realistic value should exceed the assumed one, thus a retention exceeding $80 \%$. The current framework allows for model uncertainty within the physical model used, i.e. Type I or Type II model. More analyses need to be conducted to assess the nonlinear kinetic models using Type I and Type II to assess the impact of the parameter space on the rate of fine retention thus the resilience of the dam to self-rehabilitate.

Through the dam scale numerical investigation we explored two different representations of the flow and transport of released fines through the dam embankment. It is worth noting that at the dam scale model whether single or dual populations formulation is used calls for not only several parameters but also different functional forms of the attachment kinetics, $F$. Those parameters and functional forms need to be either measured at the dam scale or upscaled from grain scale numerical investigation. In the next section we will address the physical processes at the grain scale and the mesoscale (few 10 s of centimeters).

\section{NUMERICAL INVESTIGATION AT THE GRAIN SCALE}

SOIL EROSION IN A CONCRETE-WRAPAROUND CRACK

If the shell and filter zones do not effectively fill the cracks and prevent higher flow of water, then erosion soil within the initial crack may potentially lead to dam failure, necessitating an understanding of erosion characteristics of the soil within the dam core. Detailed validation experiments and numerical simulations of soil at the micro scale are needed to better 
understand and parameterize soil properties. It is well known that different soils erode at different rates depending on factors such as grain size, soil composition, plasticity, compaction, water content, stress-history, and soil cohesion. Several experimental methods have been developed to analyze soil erosion, the most common being the hole erosion test, or "HET" (e.g., Wan and Fell 2004, Bonelli and Brivois 2008), and the jet erosion test, or "JET"(e.g., Hanson and Cook 2004). Such experiments provide data for specific types of soils, though the general constitutive relationship between erosion parameters and geotechnical or chemical soil properties remain unknown (Wan and Fell 2002).

An initial computational approach to the problem is based on sediment transport modeling. Most hydraulic sediment transport models represent both soil and fluid as a continuum (e.g., Brethour 2001, Zhao and Fernando, 2007) where the erosion domain is represented as two media. The first medium is a packed solid domain consisting of particles completely bound together and forming a solid-like structure; the second medium is a mixture of suspended particles and fluid. At the surface of the packed bed of sediments, the fluid shear stress acts to remove sediment (first medium). If the fluid stresses are high enough to break the frictional forces between the particles, they begin to migrate from the surface, forming suspended particles (second medium). The majority of sediment transport models assume that sediment motion occurs when the Shields parameter:

$$
\theta=\frac{\tau}{g d\left(\rho_{s}-\rho_{f}\right)}
$$

exceeds a critical value $\theta_{c r}$. Here $\rho_{s}$ and $\rho_{f}$ are sediment grain and fluid density, correspondingly, $\tau$ is the shear stress, and $d$ is the grain diameter. The erosion process is then the simulated by an erosion constitutive law that manifests either as the mass or momentum exchange terms at the eroded boundary. In this case, the packed sediments are not simulated, and the solid domain is represented as a moving boundary. Bonelli et al. (2006) proposed a phenomenological piping erosion model using asymptotic analysis assuming that: (1) sediment concentration is low and does not affect density, velocity and stresses, (2) the flow is quasi steady, and (3) interface velocities are low and do not contribute to the inertia. Using these assumptions, they showed that dimensionless radius of the hole can be estimated as an exponential function of dimensionless time and initial and critical stress, when the hole is progressively eroding as:

$$
\frac{R(t)}{R_{0}}=1+\left(1-\frac{\tau_{c}}{\tau_{0}}\right)\left(e^{\frac{t}{t_{e r}}}-1\right)
$$


where $R(t)$ is hole radius at time $t, R_{0}$ is the initial radius at time zero, $\tau_{c}$ is critical shear stress, $\tau_{0}$ is shear stress at time zero. Characteristic erosion time scale is defined as $t_{e r}=\frac{2 L \rho_{s}}{k_{e r} \Delta h}$, where $k_{e r}$ is erosion coefficient, $\mathrm{L}$ is a characteristic geometrical length of the system and $\Delta \mathrm{h}$ is the head drop. Furthermore, the dimensional flow rate is given by:

$$
Q^{*}=\frac{Q(t)}{Q_{0}}=\left(\frac{R(t)}{R_{0}}\right)^{5 / 2}=\left[1+\left(1-\frac{\tau_{c}}{\tau_{0}}\right)\left(e^{\frac{t}{t_{e r}}}-1\right)\right]^{5 / 2}
$$

This model can be used for both interpreting HET experiment as well as predicting crack growth if soil properties are known from HET experiment. The significant advantage of this method for interpreting HET experiment is that the final diameter of the hole does not need to be measured to find the soil erosion coefficient $k_{e r}$. Assuming that erosion coefficient $k_{e r}$ and critical stress $\tau_{c}$ are known from HET experiment for particular soil type, it is straightforward to estimate crack growth in time from equation (16). While this model was applied to numerous HETs experiments and was found to reproduce HET test results quite well it is not generally applicable for other erosion processes, e.g. JET experiment. Another approach is to introduce a diffusion term (lift force) for the solid phase (Brethour, 2001). In the current approach both solid domain and domain with a mixture of fluid and suspended sediments are simulated simultaneously as one system. The diffusion term (lift force) is introduced as $\mathrm{F}=\nabla(\mathrm{k} \nabla \mathrm{C})$, where the diffusion coefficient $\mathrm{k}$ depends upon the shear stress and critical shear stress difference $\left(\tau-\tau_{c}\right)$, and $C$ is the particle phase concentration.

A challenge arises when simulating the flow of the solid laden fluid. Correct representation of the general rheological behavior of the solid and fluid mixture as well as transition from solid to fluid-like behavior is a very complicated problem. The common approach is to assume a shear stress-shear rate constitutive relationship:

$$
T=-\left(\lambda-\frac{2}{3} \mu\right)(\nabla \cdot u) I+\mu S
$$

where $T$ is the shear stress, $S$ is the shear rate, and $\mu$ and $\lambda$ are shear and bulk viscosities, respectively. One approach to simulate fluid-particle suspensions (or mud flow) is to treat them as thixotropic yield stress fluids or Bingham fluids (Chanson et al. 2006). The granular bed deforms in the region in which the shear stress exceeds a certain value (yield stress). In these models the effective viscosity is determined by the shear stress and the shear rate as: 


$$
\mu_{e f f}=\left\{\begin{array}{c}
\mu_{0}+T_{0}, T \geq T_{0} \\
\infty, T<T_{0}
\end{array}\right.
$$

where $T_{0}$ is the constant yield stress, and $\mu_{0}$ is the initial shear viscosity. In another approach (Zhao et al. 2007) the solid shear viscosity $\mu_{s}$ is represented as a sum of frictional $\mu_{\text {fric }}$, collisional $\mu_{c o l}$ and kinetic viscosities $\mu_{\text {kin }}$ :

$$
\mu_{s}=\mu_{\text {fric }}+\mu_{\text {col }}+\mu_{k i n}
$$

and an additional drag term dependent on the solid volume fractions is included in the momentum equations to represent solid-like behavior. Both approaches (Equations (19) and (20)) have been incorporated into the numerical code used herein to investigate soil erodibility within the crack. To represent the settling of the sediment particles due to gravity we estimate the relative slip velocity between fluid and particles as

$$
u_{\text {slip }}=\frac{d^{2}}{18 \mu f_{\text {drag }}} \frac{\nabla P\left(\rho_{s}-\rho_{f}\right)}{\rho}
$$

where drag term ( $f_{d r a g}$ ) is function of the Reynolds number

$$
f_{\text {drag }}=\left\{\begin{array}{c}
1+0.15 R e^{0.687} \text { if } R e \leq 1000 \\
0.0175 R e \quad \text { if } R e>1000
\end{array}\right.
$$

For densely packed fluidized beds, the drag equation (21) also takes into account volume fractions occupied by fluid and particles.

We applied the numerical model described above to investigate erosion processes for HET and JET experiments. Figure 32 shows an erosion simulation within a crack of maximum and minimum width of $8 \mathrm{~mm}$ and $6 \mathrm{~mm}$, respectively. The flow is caused by a pressure gradient of $27 \mathrm{kPa} / \mathrm{m}$ (a hydraulic gradient of $2.75 \mathrm{~m} / \mathrm{m}$ ). In this simulation we assumed that critical shear stress was $8 \mathrm{~Pa}$. This numerical configuration is close to FTHET010 experiment conditions considered in Bonelli et al. (2006). Figure 33 shows results of simulations of JET experiment. The $6.4 \mathrm{~mm}$ diameter submerged hydraulic jet was positioned initially $30 \mathrm{~cm}$ above the sample surface. The velocity at the jet nozzle was $6.6 \mathrm{~m} / \mathrm{s}$. As a submerged jet erosion test progresses with time, the scour surface in the zone of the impinging jet erodes away from the jet nozzle 
until an equilibrium depth is reached. The HET and JET methods yield significantly different estimates of the erosion coefficient and critical stress (Wahl et al. 2008). The numerical experiments described above are performed for the same type of soils. We estimate the factor of difference for erosion coefficient between the JET and HET experiments to be 0.06 which agrees with experimental data (Regazzoni 2008). It should be noted that JET method is easier to perform, and it is applicable to a wider range of soils. Therefore, establishing connection in interpretation between these two tests is an important task. Direct numerical simulation of soil erosion with the continuum approach described above can be used to get more insight on the difference between JET and HET data. Also, the model assumes empirical constitutive laws for soil behavior that need further improvements and validation. In the scenario considered so far, it is assumed that the hole is progressively eroding and eventually will lead to the dam failure, if the filters do not prevent further erosion. The issues related to the filter design are considered in the next section.

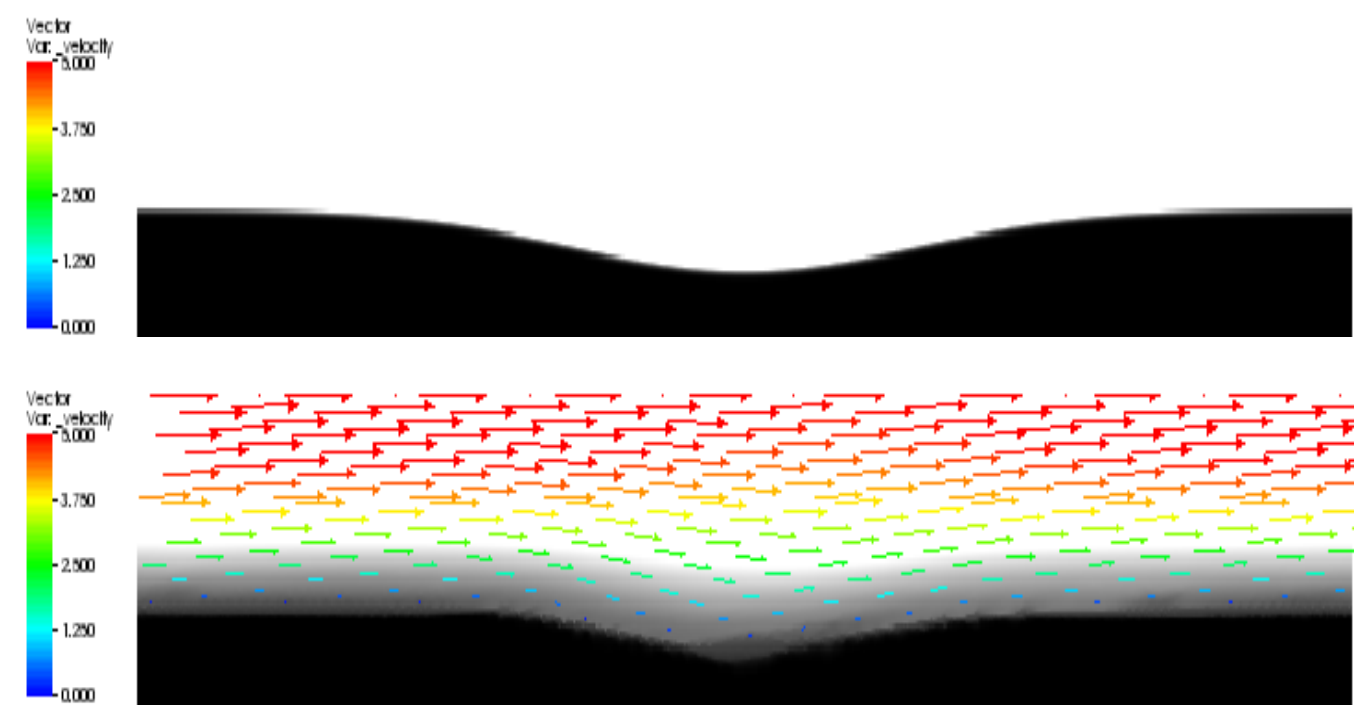

Figure 32. Numerical simulation of multiphase flow in an interfacial gap between concrete and soil (HET experiment). Top frame: initial conditions, bed not subject to erosion. Bottom frame: bed is subject to horizontal flow.

\section{BEHAVIOR OF GRAVEL IN FILTER ZONES}

Granular filters are one of the most important protective design elements of large, zoned embankment dams. The main role of filters is to prevent movement of the core materials past the filter zones and to lower the phreatic surface so that water safely seeps through the embankment at the downstream toe. Design of the size distribution of particles within the filter is based on a large body of empirical, physical experiments. Numerical rather than empirical determination of filter zone gradations is nontrivial. For example, the particle size distribution of the filter should contain small enough particles to have small void spaces and prevent 
erosion. However, it is well known that seepage flow within a coarsely graded filter material can erode finer particles, thus generating a "suffusion" process that may over time compromise the performance of the filter. Also, clogging of the filter may occur, causing loss of the filter's ability to remove water from protected soil.

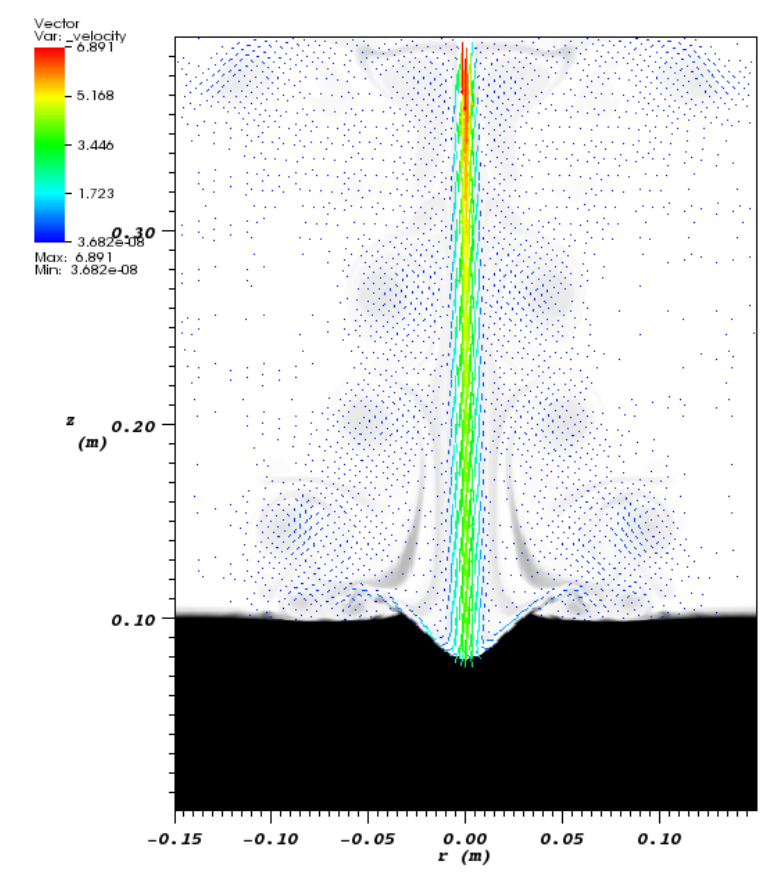

Figure 33. Numerical simulation of JET experiment.

Existing filter designs criteria are, in most cases, empirically derive (e.g., USACE Engineer Manual 1110-2-1901, Fell et al. 2005). Some of these criteria are listed below.

Clogging criterion: $\quad D_{15}^{\text {filter }} / D_{85}^{\text {soil }} \leq 5$

Permeability criterion: $\quad D_{15}^{\text {filter }} / D_{15}^{\text {soil }} \geq 5$

where $D_{15}$ and $D_{85}$ are the diameters of a soil mixture which correspond to cumulative weight percentage of $15 \%$ and $85 \%$ in a particle size distribution, respectively. These criteria are derived for filters that are operated during normal conditions. During dynamic loading due to earthquakes or explosions the filter can be deformed homogeneously with associative dilatancy, as well as localized shear bands with even higher void ratios can be created. Numerical modeling holds promise as a complimentary approach for optimizing the selection of filter components. Numerical continuum formulations of flow through porous media are one way to approach such problem. Continuum approaches are often heavily dependent on a few 
unknown parameters such as soil permeability. The physical background of such parameters typically depends on the localized effects of the flow and pore geometry. Thus, detailed simulations of the flow at the grain-scale can provide the necessary physical parameters for the continuum formulations. An example of such direct simulations of fluid flow through the filter layer that consists of spherical particles is illustrated in Figure 34.

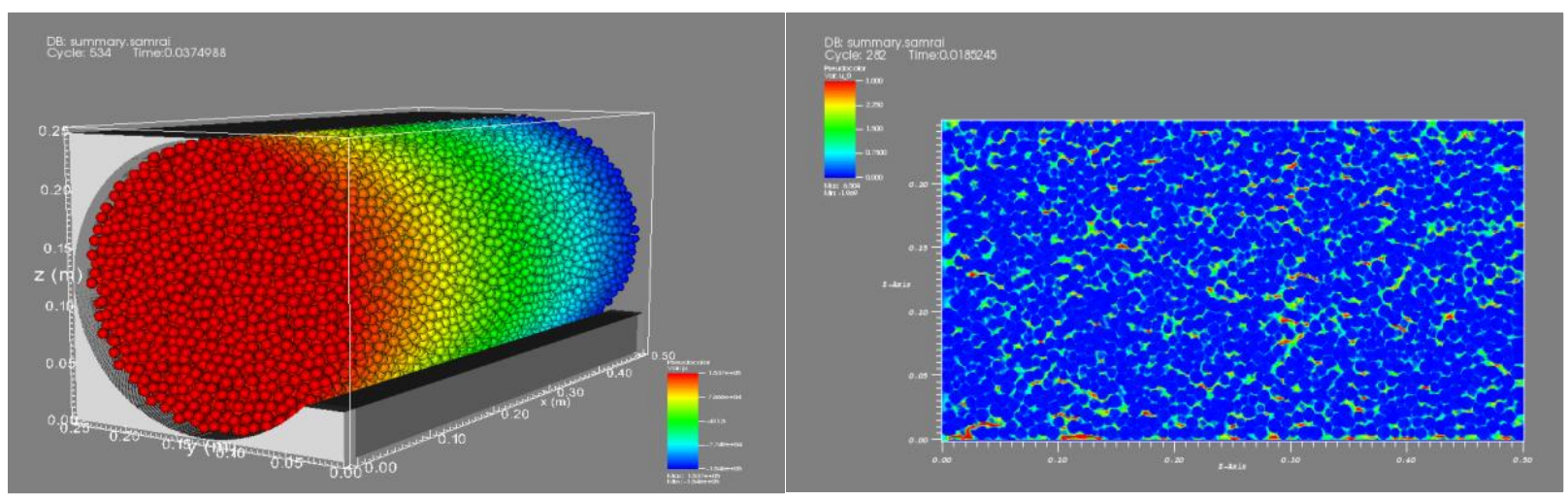

Figure 34. Simulation of the fluid migration through the gravel filter. Hydraulic pressure drop (left panel) and fluid velocity (right panel).

Another example of how local inhomogenities in the filter packing could affect its permeability is shown in Figure 35. Two different configurations of randomly packed spheres with the same porosity of $42 \%$ are considered (Figure $35 \mathrm{a}, \mathrm{b}$ ). In the first configuration the packing anisotropy in the vertical plane is introduced by removing few particles from initial randomly packed structures with porosity of $36 \%$. In the second configuration, the anisotropy is introduced in the horizontal plane in the same way. We estimate the maximum difference in pore pressure between these two cases is $22 \%$ (Figure $35 \mathrm{c}$ ).
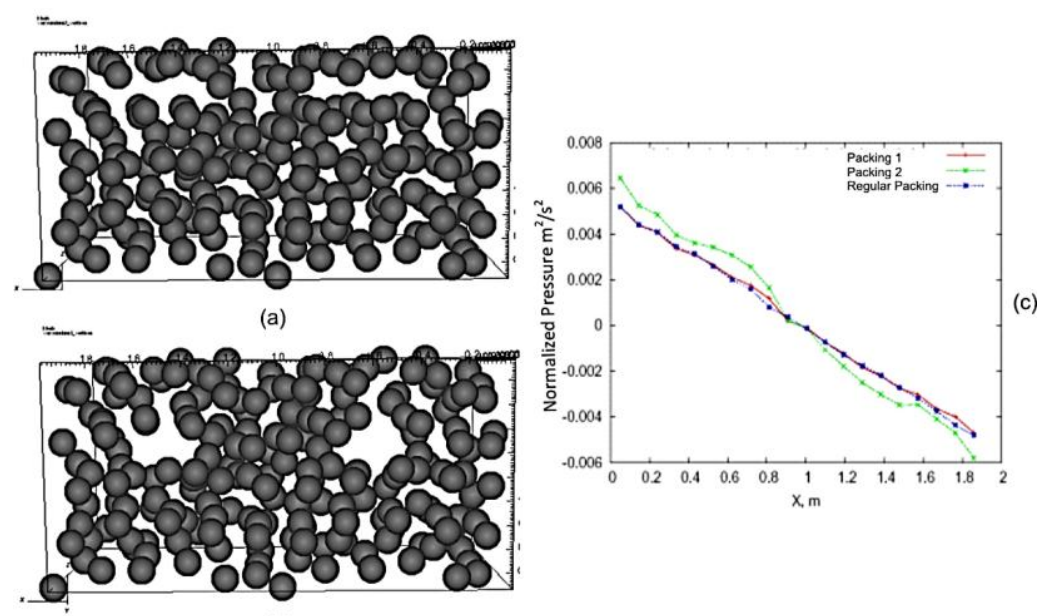

Figure 35. Simulation of the fluid migration through the gravel filter with local inhomogenities in packing in the vertical plane (a) and horizontal plane (b), difference in hydraulic pressure drop (c). 
When there is a significant opening between concrete and both filter and core sections, the coarse grains and gravel become detached from the filter and slide into the crack or are transported away by the flowing water. An example of gravel migration and sedimentation through a crack is illustrated in Figure 36. Figure 36 shows a computational domain of $0.55 \mathrm{~m} \mathrm{x}$ $0.13 \mathrm{~m} \times 0.13 \mathrm{~m}$. Here an initial layer of spherical gravel particles of $1 \mathrm{~cm}$ in diameter is placed between two water tight layers, and an initial hydraulic pressure drop through a crack of 5 $\mathrm{kPa} / \mathrm{m}$ is applied. As the fluid moves through a crack, gravel particles become detached from the filter layer and are transported downstream. Under certain conditions, the gravel could fill the crack and prevent further erosion.

(a)

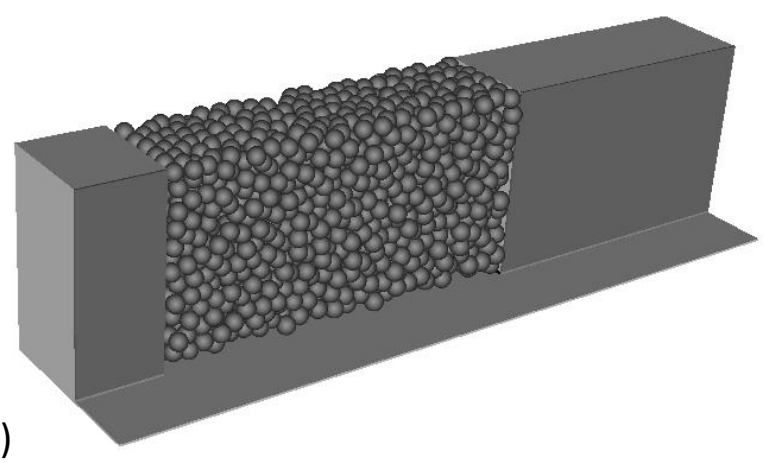

(b)

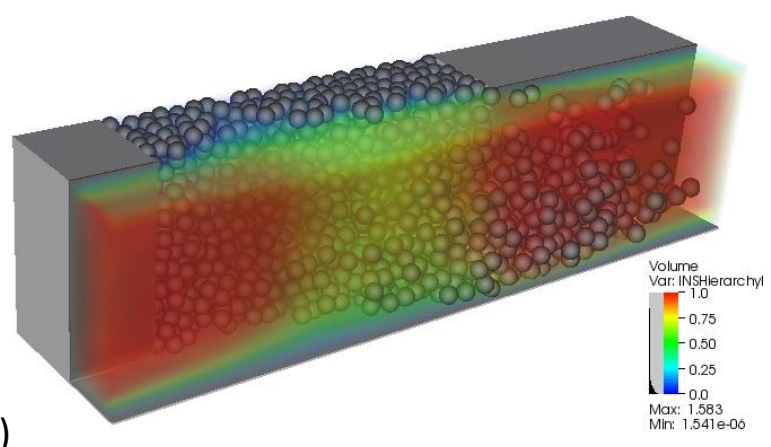

Figure 36. Gravel motion through a crack. (a) Initial setup. (b) Particles and flow field at 0.5 seconds. The magnitude of a velocity field is shown in color.

These simulations employ the Lagrange multiplier technique (Glowinski et al. 1999, Sharma and Patankar, 2005) and a stationary Eulerian grid with adaptive mesh refinement (AMR) to model fluid flow. Each particle is explicitly resolved on the Eulerian grid as a separate domain, using solid volume fractions. The fluid equations are solved throughout the entire calculation domain; however, Lagrange multiplier constraints are applied inside the particle domain such that the fluid within any volume associated with a solid particle moves as an incompressible rigid body. Mutual forces for the fluid-particle interactions are internal for the system. The particles interact with the fluid via fluid dynamics equations, resulting in implicit fluid-rigid-body coupling relations that produce realistic fluid flow around the particles (i.e., no-slip boundary conditions). In addition, particle-particle interaction is implemented using the DEM method (Cundall and Strack 1979) with frictional, inelastic contact forces. This method is flexible enough to handle arbitrary particle shapes and size distributions and does not require extra parameterizations for fluid-particle interactions. This approach is suitable for developing parameterizations and constitutive laws for the continuum (or multiphase) approach described in the previous section.

Additional computational complications arise when predicting erosion within a solid matrix structure consisting of gravel, sand, and fine particles (or clay). For example, the suffusion 
process, when finer soil particles are detached by seepage flow from the solid matrix, may ultimately lead to the filter failure. To our knowledge, the general erosion laws for this process have yet to be derived, and only a few attempts have been made toward a better understanding of numerical and experimental modeling of the suffusion process (Bonelli and Marot 2008). The problem has similarities with the dialysis process used for blood filtration (e.g., Kleinstreur et al. 1983) where molecules diffuse from high concentration to low concentration through a semi-permeable membrane. However, unlike dialysis, during erosion, the particles can be detached from the solid matrix (contrasting to a fixed membrane of known permeability in dialysis). It is clear that the continuum formulation cannot model this class of problem. At the same time, the discrete representation of each grain ranging from finer particles (clay) to gravel-scale is computationally intensive, requiring large computational resources. We anticipate that careful experimentally validated discrete simulations of the gravel together with continuum representation of finer particles described in the previous section are needed to study the internal erosion process in a gravel/sand/clay mixture and the trapping of the particles into filter zones of embankment dams.

\section{FILTERS CLOGGING CRITERIA}

Filters are mainly designed using empirical criteria. These criteria have been proposed by researchers as the result of a correlation between different base soil ('base soil' refers to any material of the dam or foundation that is able to suffer internal erosion and must be protected, for example, the clay core of an embankment dam) and filter variables that produce a satisfactory behavior if they are tested in a laboratory under different conditions. Some of these empirical criteria are:

$$
\begin{array}{ll}
\frac{D_{15}^{\text {filter }}}{D_{85}^{\text {soil }}} \leq 5 & \text { USBR, } 1955 \text { and } \\
\frac{D_{15}^{\text {filter }}}{D_{85}^{\text {soil }}} \leq 9 & \text { sherard et al., } 1984
\end{array}
$$

where $D_{15}$ and $D_{85}$ are the diameters of a soil mixture which correspond to cumulative weight percentages of $15 \%$ and $85 \%$ in a particle size distribution of filter and soil.

Indraratna et al. (2007) proposed to use constriction-based criterion $D_{C 35} / d_{85} \leq 1$, where $D_{C 35}$ is the equivalent constriction diameter. These empirical criteria indirectly take into account all the factors affecting filtration but are only applicable to the range of soils tested and depend on testing methods, definitions of failure, etc. Therefore, additional experimental data and numerical simulations are needed to evaluate clogging criteria in filter layers. Interfaces 
between concrete and earthen layers of embankment are most susceptible to develop inhomogeneity under hazardous conditions due to impedance mismatch between materials. Consequently, empirical clogging criteria developed for bulk materials may not be applicable under large deformation and need to be independently validated. Numerical modeling holds promise as a complimentary approach to address this problem.

\section{GRAIN SCALE MODELING}

We performed extensive investigations to establish the validity of our computational modeling approach by comparing simulation results with well-established empirical and analytical models describing flow through packed beds. The results of comparison against Ergun equation for flow through stationary beds (periodic and cubic packing) can be found in Kanarska et al. (2011).

The majority of sediment transport models assume that sediment motion occurs when Shields parameter (equation (15)) exceeds a critical value $\theta_{c}$. This fact was confirmed in experiments by many researchers (Shields 1936, Meyer-Peter and Muller 1948, Soulsby 1997, Miedema 2008). However, the value of threshold parameters varies among them and may reach some time a factor of two or even more (Buffington and Montgomery 1997, Ouriemi et al. 2009). A generalized erosion law in dimensionless form can be represented as:

$$
\frac{q}{q^{*}}=A\left(\theta-\theta_{c}\right)^{c}
$$

where, on the left side, the actual mass flux $q$ is dimensionalized by the mass flux: $q^{*}=\gamma \rho_{f} \sqrt{g d}$, where $\gamma$ is the specific gravity, $\rho_{f}$ is the fluid density and $\mathrm{d}$ is the grain diameter. Many experimental and numerical studies have been performed and published to confirm the appropriate choice of the empirical constants $A$ and $c$. An order of factor of difference for parameter $A$ and a factor of two for constant $c$ were found. For example, Wan and Fell (2004), Bonelli and Brivos (2008) assumed a linear function of critical shear stress in erosion law (C=1). Lobkovsky et al. (2008) found that for $C=1.89 \pm 0.25$ and $A=0.11 \pm 0.03$, equation (17) fits well their experimental data. Meyer-Peter and Muller (1948) proposed to use $A=1.5$. Wong and Parker (2006) found the following values $A=4.93$ and $C=1.6$. In general, these parameters depend on the flow and the particle properties and therefore need to be investigated in more detail. Shields (1936) ran a series of flume experiments with water flowing over flat beds of sands. As the applied shear stress is increased, a critical shear stress is reached at which grains will begin to move. He plotted the results of his experiments in a dimensionless form on what is now known as the Shields diagram (Figure 37). 


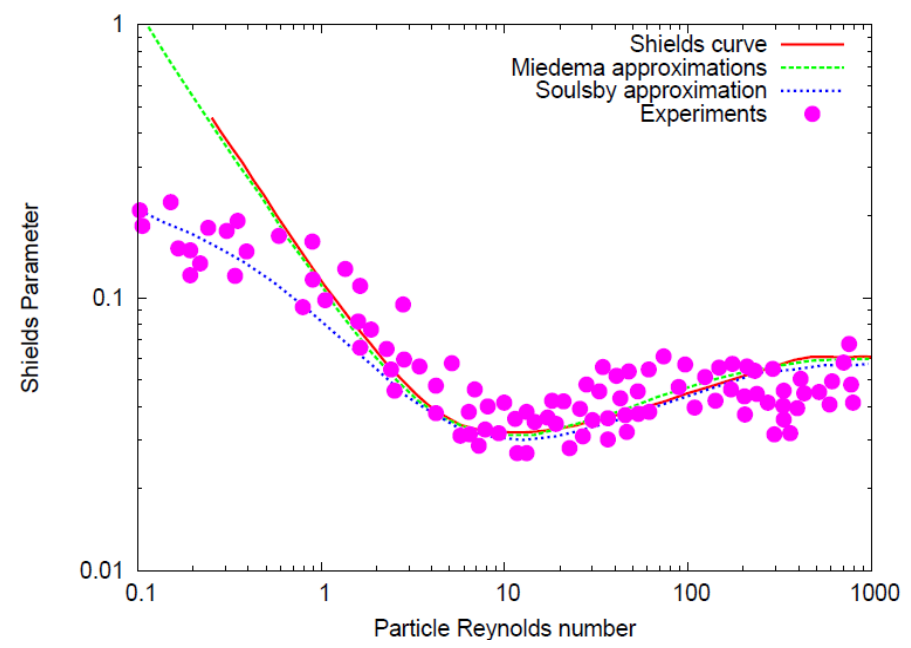

Figure 37. Shields diagram (adapted from Miedema, 2008) shows relationship between dimensionless shear stresses (Shields parameter) and particle Reynolds number. Shields curves proposed by different authors (Soulsby 1997; Miedema 2008) are shown. Please note that Shields curves are derived for non-cohesive soils.

Shields (1936) obtained a relation (the well-known Shields curve) between particle Reynolds number $\operatorname{Re}_{p}=u_{*} d / v$, where $v$ is the kinematic viscosity, $d$ is a particle diameter, $u_{*}$ is the critical shear stress velocity. The value of the critical stress depends on the size and density of the particles as well as on their shape, packing and the cohesive forces acting between particles.

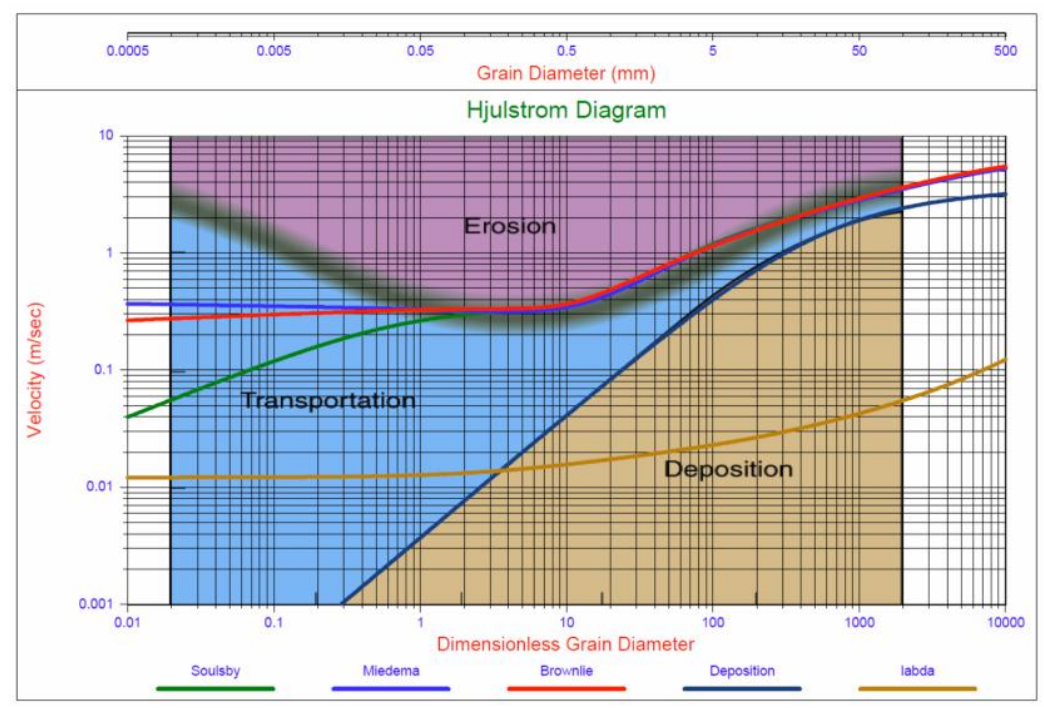

Figure 38. Hjulstrom and Shields diagrams converted to mean flow velocity (adopted from Miedema, 2008). Hjulstrom diagram (bold gray line and dark blue line) shows the relationship between stream velocity and particle erosion, transport and deposition. Bold gray line shows erosion velocity derived by Hjulstrom (1935) for cohesive sediments. 
Another parameter that characterizes soil erosion is erosion (or pickup) velocity that is defined as the minimum flow velocity required for picking up a particle initially at rest. It is closely related to the critical shear stress. Erosion velocity, similar to the shear stress velocity, is a complex function of many variables, including particle properties (size, shape, density, electrostatic behavior) and fluid properties (density, viscosity). Figure 38 shows several key concepts about the relationships between erosion, transportation, and deposition of particles known as the Hjulstrom diagram (Hjulstrom, 1935). Approximations of Shields curve converted to mean flow velocity by Brownlie (1981), Soulsby (1997), and Miedema (2008) approximations are also shown in Figure 38. For particle sizes where friction is the dominating force preventing erosion, the curves follow each other closely, and the required velocity increases with particle size. However, for cohesive sediments, mostly clay and silt, the erosion velocity increases with decreasing grain size, as the cohesive forces are relatively more important when the particles get smaller. The critical velocity for deposition, on the other hand, depends on the settling velocity, and that decreases with decreasing grain size. The Hjulstrom curve, plotted by the bold gray line in Figure 38, shows that sand particles of a size around $0.1 \mathrm{~mm}$ require the lowest stream velocity to erode.

However, many other researchers found significant deviations from Shields and Hjulstorm diagrams (see, for example, overview in Ouriemi et al. 2009). Here we attempt to clarify these uncertainties with the help of numerical experiments using the grain-scale model (Kanarska et al. 2011). First, we establish a set of particle-scale configurations to study response of a granular bed to forcing by a fluid which flows over the crack surface for different particle sizes, shapes, and environmental conditions. Then we compare our results with a few recent experiments of Dasani et al. (2009), Bohling et al. (2009), and Lobkovsky et al. (2008) as well as empirical and theoretical approximations related to Hjulstrom and Shields diagrams. A general constitutive law for erosion rates is established and investigated for different particle properties.

\section{GRAIN SCALE SOIL EROSION SIMULATIONS}

A packed layer of soil particles (spherical or irregular, crushed particles) of initial height $h^{*}=$ $6 \mathrm{~mm}$ is being eroded by fluid flow initiated by a pressure drop equal to $1000 \mathrm{~Pa} / \mathrm{m}$ over length $L=8 \mathrm{~mm}$. The fluid has density $\rho_{f}=1000 \mathrm{~kg} / \mathrm{m}^{3}$ and kinematic viscosity of $10^{-6} \mathrm{~m}^{2} / \mathrm{s}$, the particle density is $\rho_{S}=2500 \mathrm{~kg} / \mathrm{m}^{3}$. Physical parameters of fluid and particles which we used in the numerical configurations are given in Table 3.

We used 6 sets of particle sizes (10,50, 100, 200, 400, and 800 microns) for spherical particles and 4 sets $(50,100,200$, and 400 microns) for irregular particles which were approximated by 
triangular shapes. A slice of simulations of soil erosion for 400 micron spherical and irregular particles is shown in Figure 39.

Table 3. Physical properties of particles and fluid.

\begin{tabular}{|ll|}
\hline Parameter & Value \\
\hline Particle density & $2500 \mathrm{~kg} / \mathrm{m}^{3}$ \\
\hline Fluid density & $1000 \mathrm{~kg} / \mathrm{m}^{3}$ \\
\hline Fluid viscosity & $1 \times 10^{-3} \mathrm{~N} / \mathrm{m}^{2}$ \\
\hline Coefficient of friction & 0.5 \\
\hline Stiffness & $5-30 \mathrm{kN} / \mathrm{m}$ \\
\hline Particle sizes & $10-800$ microns \\
\hline Particle shapes & Spherical, triangular \\
\hline Number of particles & 400 \\
\hline Porosity for spherical particles & 0.14 \\
\hline Porosity for irregular particles & 0.34 \\
\hline
\end{tabular}

We slowly increased flow capacity starting from zero in simulations. When the water is flowing over a bed, there will be a certain velocity (erosion velocity mentioned earlier) at which the solid particles will be dislodged from their resting positions. This velocity is related to the equivalent stress as critical shear stress or more commonly as critical shear stress velocity $u_{*}$. When we detected, in simulations, that particle begin to detach from the surface, we recorded both the averaged distribution of shear stresses $\tau(z)$ and the velocity profile $u(z)$ (Figure 40). Figure 40 shows that the fluid shear stress inside the particle bed is very small and the maximum value of shear stresses lies at the particle bed surface. Based on maximum shear stresses at the moment of particle detachment from the surface we estimate the critical shear stress velocity as:

$$
u^{*}=\sqrt{\tau / \varrho}
$$

At the initial stage the flow in a crack has relatively low Reynolds number ( $\operatorname{Re}<5000$, where $u$ is the flow mean velocity) and our velocity profile is close to the parabolic one at the moment of particle detachment. Once erosion begins, a small crack forms and grows into a fissure. Therefore, at later times during erosion, the flow regime can be changed from laminar to turbulent, since the characteristic length and the mean flow increase. In this case, quantitative analysis of the flow parameters is quite complicated. However, because we have the possibility to calculate shear stresses directly from the numerical simulations, it will be shown that we are able to derive a general erosion law that depends on shear stresses directly rather than the mean flow velocity or hydraulic pressure gradient. 

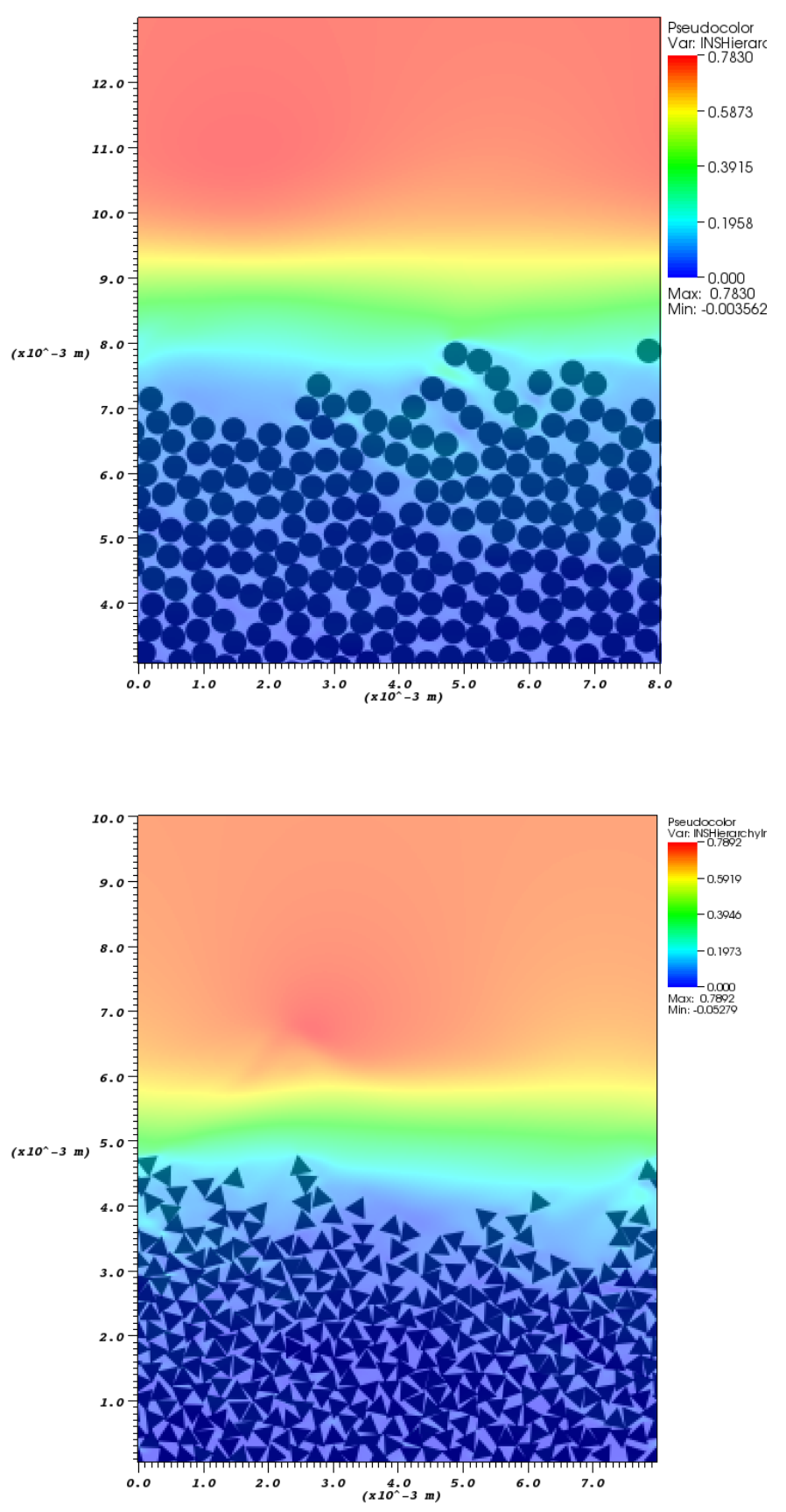

Figure 39. Simulations of erosion of $\mathbf{4 0 0}$ micron particles (spheres and irregular particles.

It is a known fact that the critical shear stress for laminar flow in a crack (Potter and Wiggert, 2008) can be calculated as:

$$
\tau=6 U \mu / H
$$


where $U$ is the averaged velocity in a crack, and $\mathrm{H}$ is the crack width. These values are also presented in Table 4. We found that the critical stresses measured directly slightly exceed critical stresses estimated from the mean parabolic profile. Even for hydraulically smooth flow (i.e., particle Reynolds number $\sim 5$ ), the rough surface of the granular bed yields a fluctuating local fluid stresses (see Figure 41). This may cause discrepancy between the shear stresses based on parabolic velocity profile and measured directly (Table 4. Numerical results from simulations with spherical particles.) and also explain some deviations from experimental data discussed below.

We compare our findings listed in Table 4, and Table 5 with the experimentally derived critical shear stress velocities performed by Bohling (2009). It is worth noting that the threshold curve for non-cohesive sediments is based on a modified Shields' (1936) curve according to Soulsby (1997).
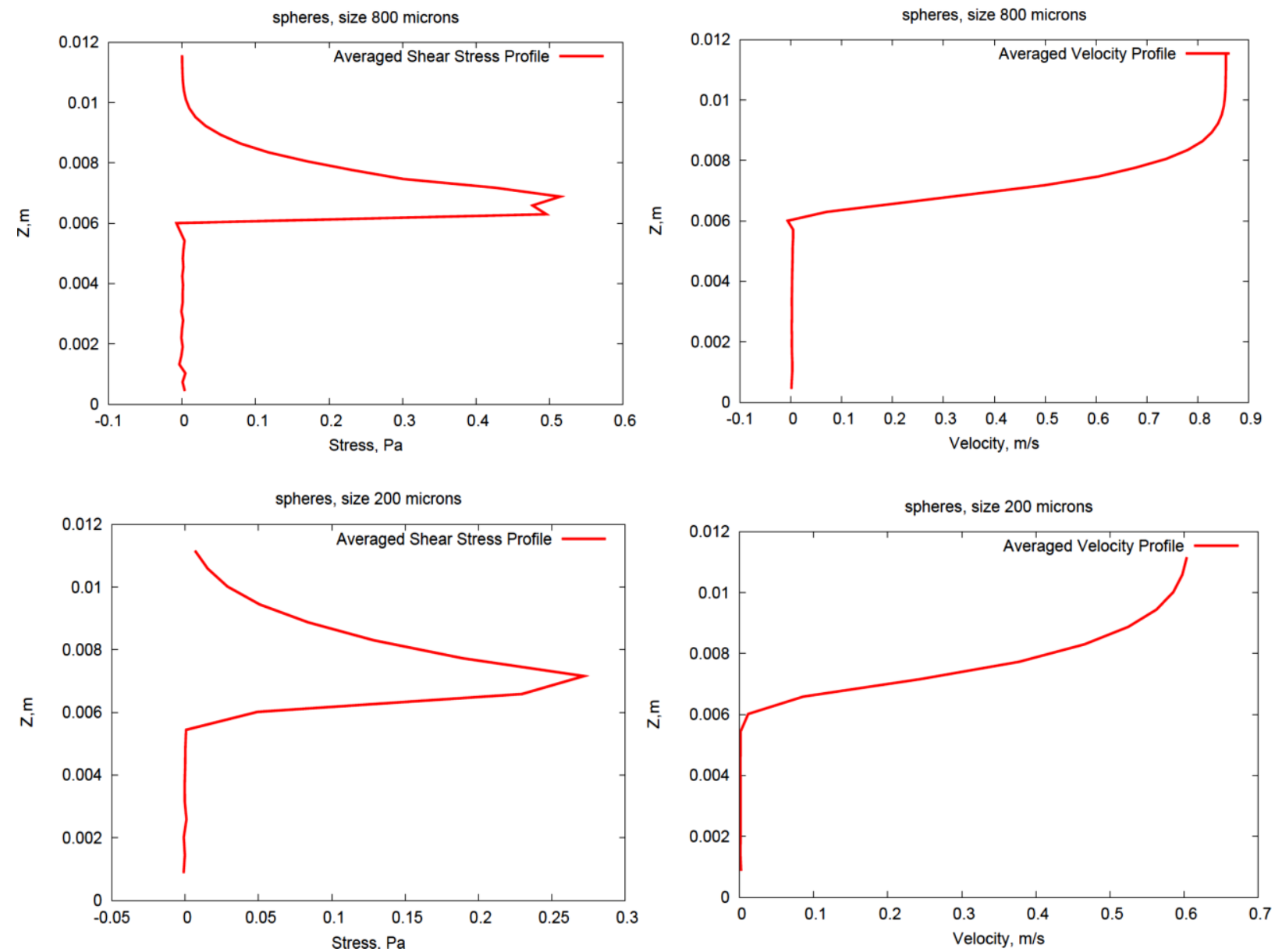

Figure 40. Examples of stress and averaged velocity profile for spheres of size $\mathbf{8 0 0}$ and $\mathbf{2 0 0}$ microns at the moment of particle detachment from the surface. 


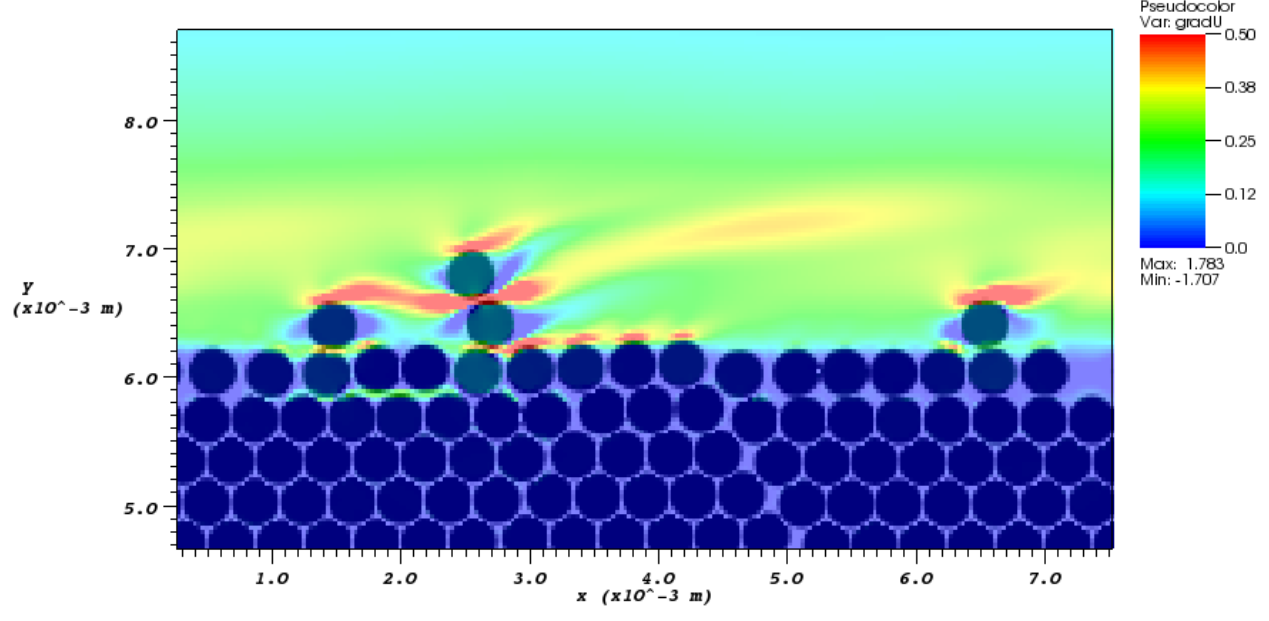

Figure 41. Shear stress distribution in simulations. Local heterogeneities in geometry of granular bed result in discrepancy between mean shear stress and local shear stress even in laminar regime.

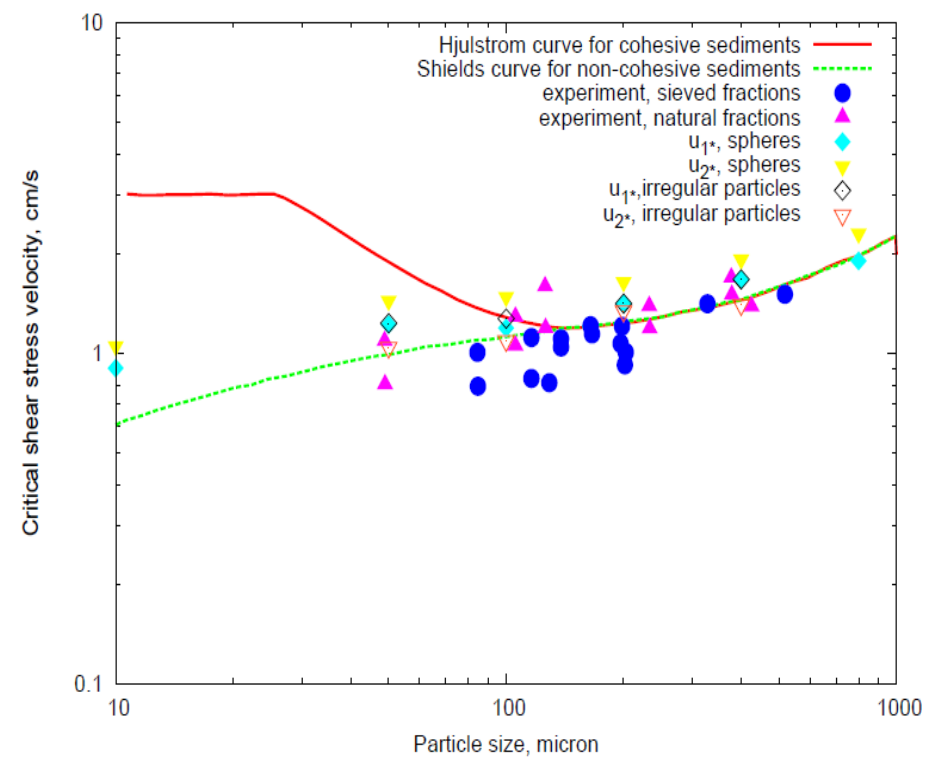

Figure 42. The experimentally derived critical shear stress velocities in comparison to correlations between critical shear stress velocity and grain size from literature. The symbols distinguish between the different used sample types sieved sediment fraction and naturally composed sediments (Bohling et al. 2007). The threshold curve for non-cohesive sediments is based on a modified Shields (1936) curve according to Soulsby and Whitehouse (1997). The curve for cohesive sediments is an adjustment to the Hjulstrom (1935) plot given by Zanke (1977). This graphic is adopted from Bohling et al. (2007) paper. Irregular shapes are also plotted.

The curve for cohesive sediments is an adjustment to the Hjulstrom diagram (Figure 38). The overall agreement with experimental data is good, however the critical velocities obtained in the simulations based on direct measurements of shear stresses is slightly higher than the 
values predicted by the empirical curves and experiments. At the same time the critical shear stress velocities based on the averaged flow properties as well as data for irregular particles match these data better. It should be noted, that an increased threshold value due to cohesive effects was not observed in the studied range of grain sizes (40-900 microns) in experiments of Bohling (2009). We found quite good correlation between experimental data of Bohling (2009) and Shields and Hjulstrom critical curves for the particle sizes in range 50-800 microns with a general tendency for critical shear stresses to decrease with decreasing the particle size. At smaller particle sizes, more likely, cohesive effects become important and needs to be taken into account.

To compare our simulation with Dasani et al., (2010) experiment that was performed in larger domain we compute the mean velocity profiles using the critical shear stress velocity obtained by simulations and assuming logarithmic velocity profile. The logarithmic velocity profile for hydraulically smooth flow is given by:

$$
\frac{u}{u_{*}}=\frac{1}{\kappa} \ln \left(\frac{u_{*} z}{v}\right)+5.5=\frac{1}{\kappa} \ln \left(9.05 \frac{u_{*} z}{v}\right)
$$

The logarithmic velocity profile for hydraulically rough surface (particle Reynolds number $>40$ ) is:

$$
\frac{u}{u_{*}}=\frac{1}{\kappa} \ln \left(30 \frac{z}{k_{s}}\right)
$$

where $k_{s}$ is the surface roughness. Detail description can be found in Potter and Wiggert, (2008). By integrating the velocity profile over the flow depth $h$ we can derive the mean flow velocity value as:

$$
u_{\text {mean }}=\frac{u_{*}}{\kappa}\left\lfloor\frac{z_{0}}{h}+\ln \left(\frac{h}{z_{0}}\right)-1\right\rfloor
$$

where $z_{0}=\frac{v}{9.05 u_{*}}$ for smooth flow and $z_{0}=\frac{k_{3}}{30}$ for rough flow. Particle Reynolds number $\operatorname{Re}_{p}$ ranges from 0.1 to 18 in our numerical experiments. Therefore we used equations (21) and (22) for hydraulically smooth flow to estimate the mean flow velocity. Results of comparisons 
between pickup velocities for different particle sizes (particle diameter 10, 50, 100, 200, 400, and 800 microns) and shapes (spherical and crushed particles approximated by spherical and triangular shapes accordingly) with Dasani et al. (2010) data are shown in Figure 43. We see that the overall agreement with experimental data is quite good. Both experiments and simulations clearly exhibit how non-spherical particles begin to be entrained by the fluid flow with smaller velocities followed by spherical particles.

Comparison of the numerical results with experiments of Bohling et al. (2009) and Dasani et al. (2010) for the shear stresses (shear stress velocities) and mean velocities show very good agreement when the particle size is between 50-800 microns for both spherical and irregular particles.

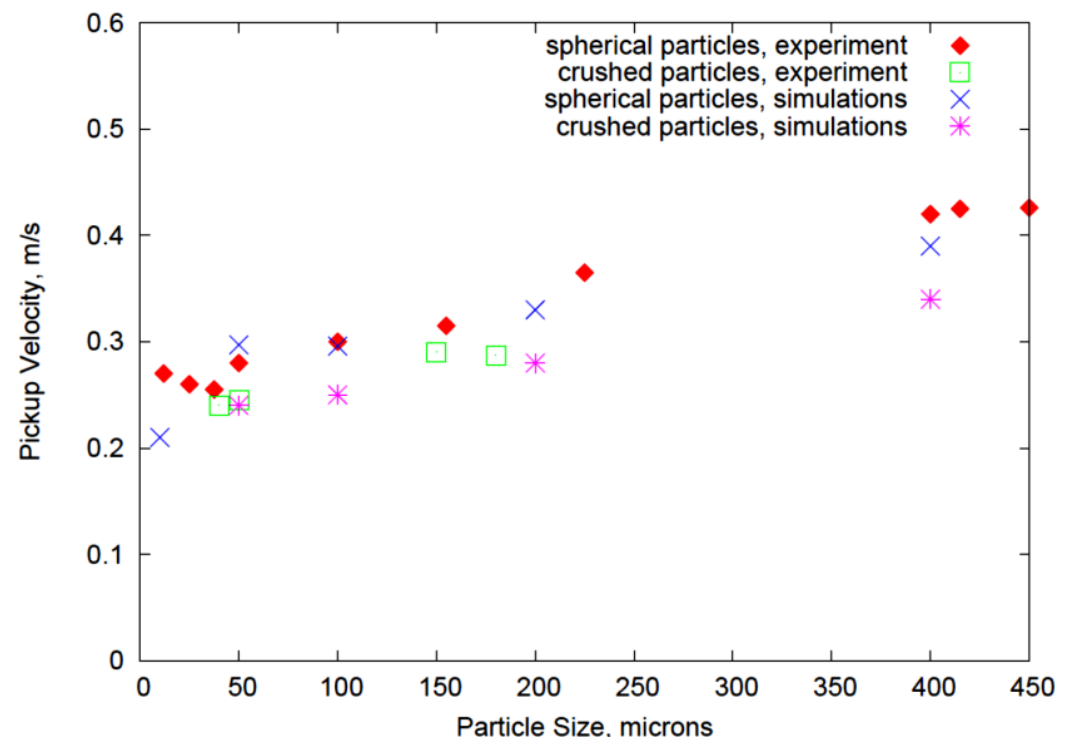

Figure 43. Comparison of pickup velocities in the experiments of Dasani et al. (2010), and the current numerical simulations.

Since we have good agreement on the threshold values with experimental data for the particle sizes of 50-800 microns, we use these numerical results to derive constitutive laws for particle mass fluxes from the crack surface. To get a general constitutive law we need to find the critical shear stress and the particle flux from the surface in a functional form. We have already described the procedure of finding the shear stresses. To find particle mass flux we estimate at each time step the particle bed position. Since we consider periodic domain in the direction of the fluid flow, we delete each suspended particle in water that reaches periodic boundary to avoid multiple particle entrances in the domain area. In this case the bed height begins to decrease with increasing time, as the test section is not fed in with particles and we can easily estimate particle bed evolution by recording maximum position of the bed. An example of particle bed evolution in time for spherical particles of radius 100 and 400 microns is shown in 
Figure 44. An example of polynomial fit for granular bed evolution of 100 and 400 micron spheres.. Please note that the bed height is normalized by the initial bed position $h^{*}$. Time is normalized by $t^{*}=h^{*} / \sqrt{\gamma g d}$. An example of polynomial fit to the particle bed evolution is a shown by curve in Figure 45 . This fit is used to get the derivative $\partial h / \partial t$ and dimensionalized particle flux $q / q^{*}$.
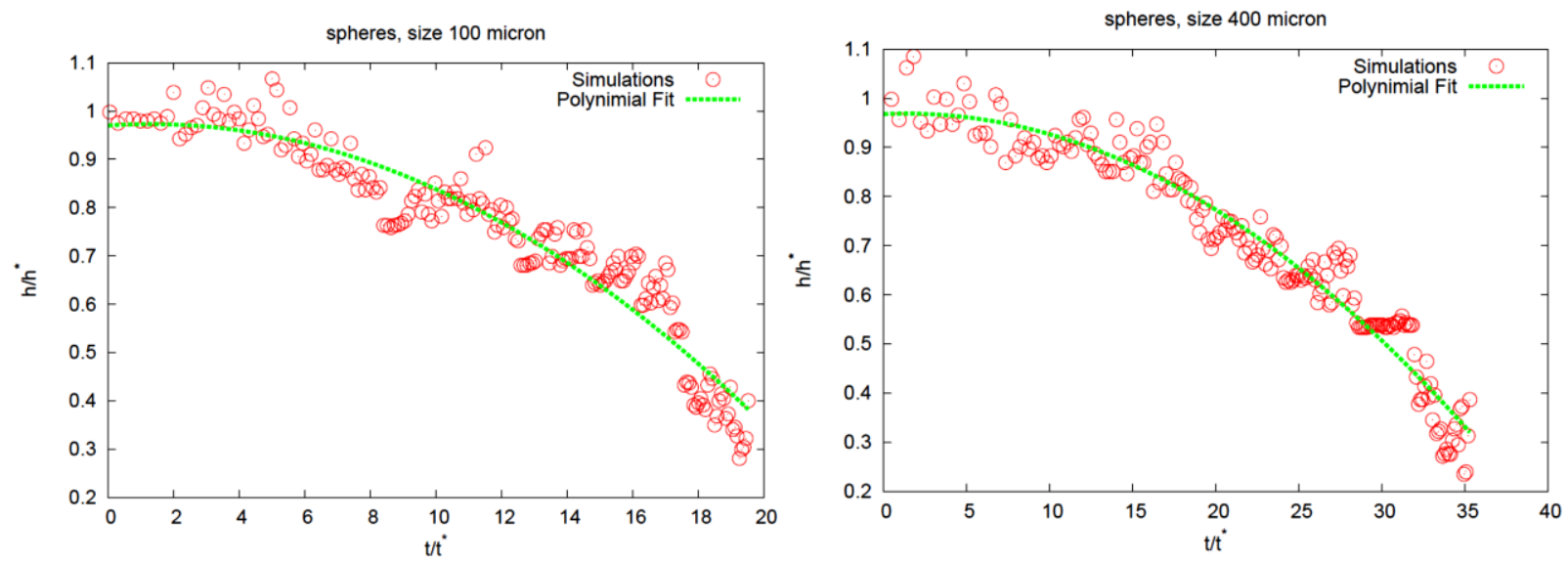

Figure 44. An example of polynomial fit for granular bed evolution of 100 and 400 micron spheres.
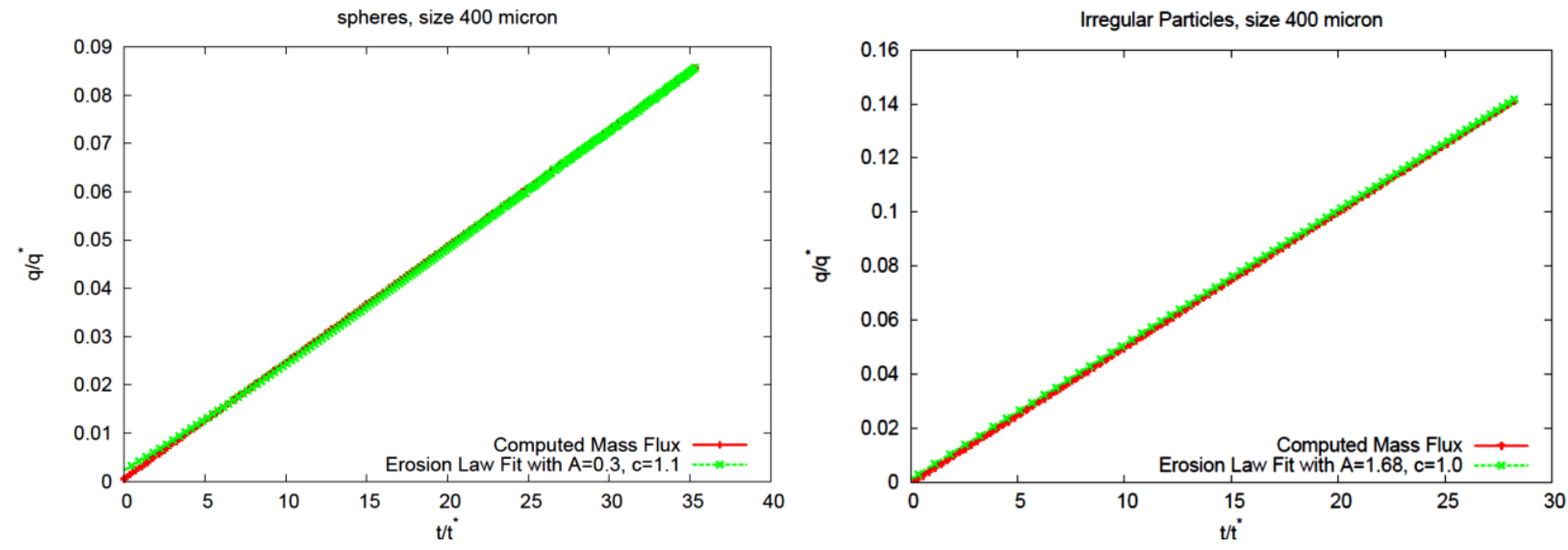

Figure 45. An example of estimation of parameters for erosion law based on numerical simulations for 400 microns particles of spherical and irregular shape.

Once the polynomial fit is found, time derivative of the bed evolution can be connected to particle flux $q$ as:

$$
q=\rho_{s} \frac{\partial h}{\partial t}
$$


Therefore the erosion law (equation (15)) can be rewritten in terms of $\partial h / \partial t$ as

$$
\frac{1}{H^{*}} \frac{\partial h}{\partial t}=A\left(\theta-\theta_{c}\right)^{c}
$$

where $H^{*}=\frac{\gamma \sqrt{g d}}{(1+\gamma)}$

Table 4. Numerical results from simulations with spherical particles.

\begin{tabular}{|lllllll|}
\hline Particle size, microns & $\mathbf{8 0 0}$ & 400 & 200 & 100 & 50 & 10 \\
\hline Critical stress, Pa, measured directly & 0.52 & 0.37 & 0.27 & 0.22 & 0.21 & 0.11 \\
\hline $\begin{array}{l}\text { Critical stress, Pa, estimated from mean } \\
\text { parabolic velocity }\end{array}$ & 0.361 & 0.28 & 0.2 & 0.14 & 0.15 & 0.08 \\
\hline $\begin{array}{l}\text { Critical shear stress velocity, } \mathbf{u}_{\mathbf{1}^{*}} \mathbf{c m} / \mathbf{s}, \\
\text { based on measured shear stress }\end{array}$ & 2.3 & 1.92 & 1.64 & 1.48 & 1.44 & 1.05 \\
\hline $\begin{array}{l}\text { Critical shear stress velocity, } \mathbf{u}_{\mathbf{2}^{*}} \mathbf{c m} / \mathbf{s}, \\
\text { based on parabolic profile }\end{array}$ & 1.9 & 1.67 & 1.41 & 1.19 & 1.22 & 0.9 \\
\hline Mean velocity, m/s, based on log profile & 0.48 & 0.39 & 0.33 & 0.296 & 0.297 & 0.21 \\
\hline $\begin{array}{l}\text { Mean velocity, m/s, based on parabolic } \\
\text { profile }\end{array}$ & 0.72 & 0.56 & 0.4 & 0.28 & 0.3 & 0.24 \\
\hline Erosion law coefficient A & 0.16 & 0.3 & 0.35 & 0.31 & 0.27 & 0.54 \\
\hline Erosion law exponent c & 1 & 1.1 & 1.15 & 1.17 & 1 & 1.2 \\
\hline Maximum Particle Reynolds number & 18.4 & 7.68 & 3.28 & 1.48 & 0.72 & 0.1 \\
\hline
\end{tabular}

Table 5. Numerical results from simulations with irregular shapes.

\begin{tabular}{|lllll|}
\hline Particle size, microns & 400 & 200 & 100 & 50 \\
\hline Critical stress, Pa, measured directly & 0.28 & 0.2 & 0.16 & 0.15 \\
\hline $\begin{array}{l}\text { Critical stress, Pa, estimated from mean } \\
\text { parabolic velocity }\end{array}$ & 0.2 & 0.18 & 0.12 & 0.11 \\
\hline $\begin{array}{l}\text { Critical shear stress velocity, } \mathbf{u}_{\mathbf{1}^{*}} \mathbf{c m} / \mathbf{s}, \\
\text { based on measured shear stress }\end{array}$ & 1.67 & 1.41 & 1.27 & 1.23 \\
\hline $\begin{array}{l}\text { Critical shear stress velocity, } \mathbf{u}_{\mathbf{2}^{*}} \mathbf{c m} / \mathbf{s}, \\
\text { based on parabolic profile }\end{array}$ & 1.4 & 1.34 & 1.09 & 1.04 \\
\hline Mean velocity, $\mathbf{m} / \mathbf{s}$, based on log profile & 0.34 & 0.28 & 0.25 & 0.24 \\
\hline $\begin{array}{l}\text { Mean velocity, } \mathbf{m} / \mathbf{s}, \text { based on parabolic } \\
\text { profile }\end{array}$ & 0.53 & 0.48 & 0.32 & 0.29 \\
\hline Erosion law coefficient $\mathbf{A}$ & 1.68 & 1.72 & 1.9 & 2.1 \\
\hline Erosion law exponent c & 1 & 1 & 1.05 & 1 \\
\hline Maximum Particle Reynolds number & 6.68 & 2.82 & 1.27 & 0.36 \\
\hline
\end{tabular}

For all particle sizes considered here, we found that erosion law power is close to 1 (ranges from 1.0 to 1.2). Coefficient $A$ in equation (33) ranges from 0.16 to 0.54 for spherical particles and from 1.68 to 2.1 for irregular shape particles. Lobkovsky et al., (2008) come to value $A=0.11$ 
\pm 0.03 for 700 microns glass beads. This is close to our value $A=0.16$ for 800 micron particles. We found about a factor of five or even more difference in erosion rates between spherical and irregular particles. This difference includes two effects: (1) the initial porosity difference between spherical packing and packing of irregular particles (initial porosity was lower for spherical particles (see Table 3), (2) difference in the drag and lift forces due to the different particle shapes. We apply gravity settling algorithm without shear velocity to get stable particle configuration. For a certain particle shape, there is a unique maximum porosity value for this configuration. Hence, it is hard to separate effects (1) and (2) from each other. Rough estimations of contribution of each of these factors can be done based on the difference in the particle pickup velocities in Figure 43, which mostly attributed to effect (2). The maximum difference between pickup velocities for spherical and irregular particles is about $20 \%$, while the difference in erosion rates is a factor of five. Therefore, we conclude that such a big difference in erosion rates is mainly due to the differences in particle compaction, which implicitly includes particle shape effects.

\section{PARTICLE AND FLUID TRANSPORT IN FILTER LAYERS}

An example of direct simulations of soil migration through the filter layer that consists of spherical particles is illustrated in Figure 46 for $D^{\text {filter }} / d^{\text {soil }}=4$ and $D^{\text {filter }} / d^{\text {soil }}=10$. Changes of permeability of filter in time show that for $D^{\text {filter }} / d^{\text {soil }}=4$ clogging of soil particles occur and soil particles are retained by the filter. For sand particles with $D^{\text {filter }} / d^{\text {soil }}=10$ the simulations predict that the particles will pass through the filter voids. These preliminary findings agree with existing empirical criteria of Sherard et al. (1984).

We initiated the investigation of soil and fluid migration through the damaged filter. We simulate two possible scenarios: case 1 - moderate damage, case 2 - significant damage to a filter zone. These two configurations were produced by applying the shear and normal force to initial homogeneous configuration. Figure 47 shows particle and fluid transport through the damaged filter. We compare solid and fluid fluxes in both configurations. A difference of factor of two in permeability was found for these two scenarios. The factor of difference in soil fluxes was even more pronounced, since in the case 1 the soil fluxes approach zero and filter selfheals due to clogging of the pores. In the case 2 the particles are continuously washed out from the existing heterogeneous structure in the filter layer (Figure 48). These first sets of simulations demonstrate the applicability of considered approach to the problem of soil clogging in filter layers. Future work will be concentrated on more detailed study of soil properties once they enter filter layers. In particular, how lateral dispersion, particle properties, magnitude of the applied hydraulic gradient as well as local heterogeneities in packing affect the clogging criteria and hydraulic conductivity of the media. 
(a)

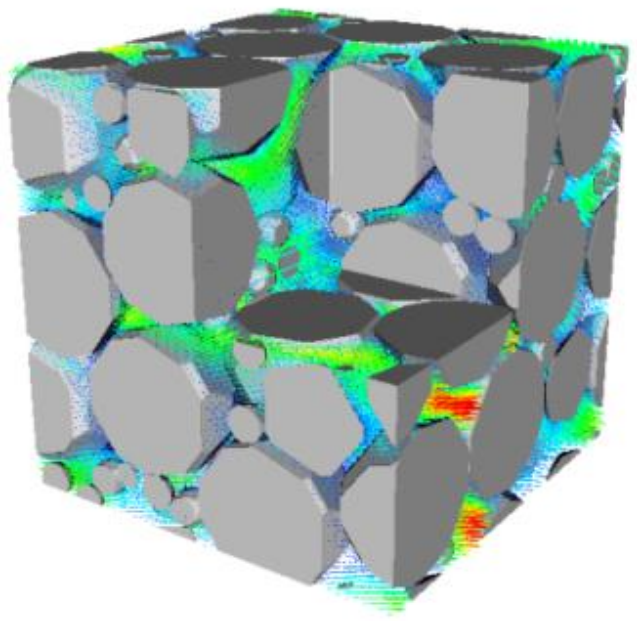

(b)

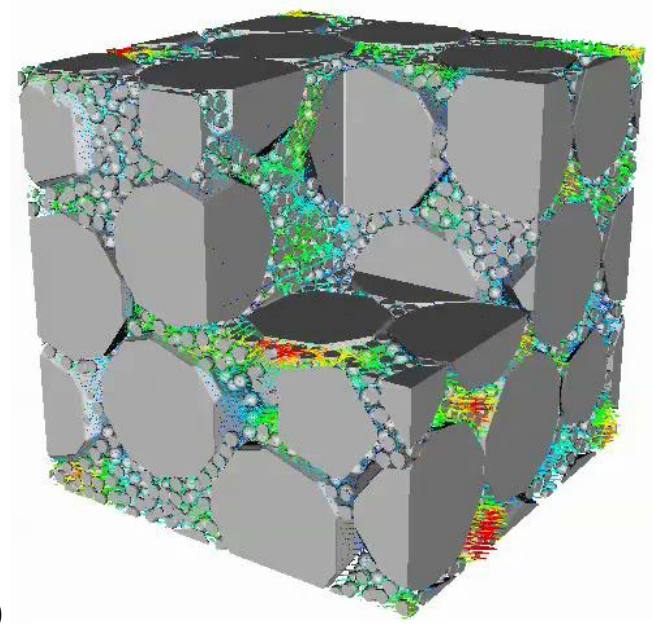

(c)

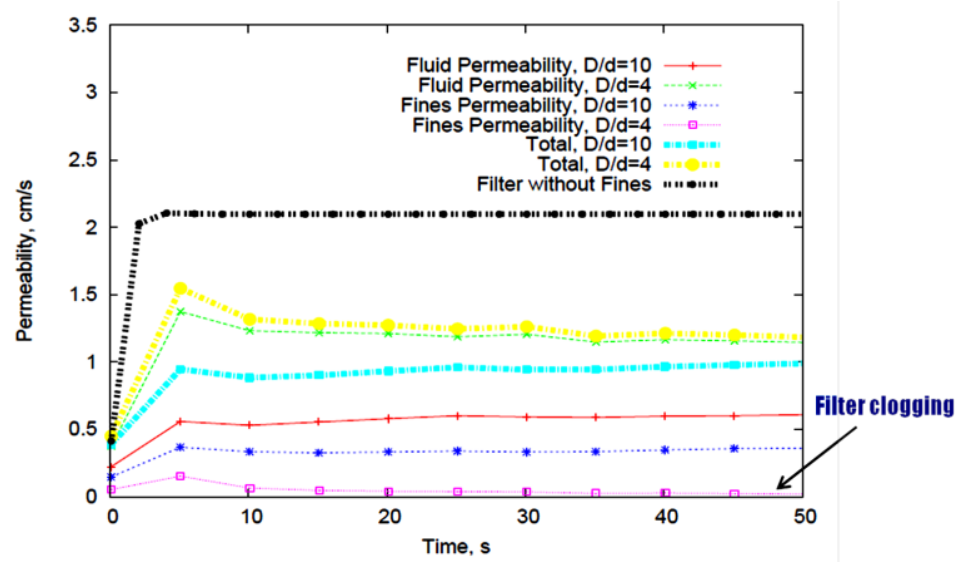

Figure 46. Code prediction for core particles migration through the filter for $D^{\text {filter }} \backslash d^{\text {soil }}=4$ (a) and $D^{\text {filter }} \backslash d^{\text {soil }}=10$ (b). Changes in permeability of the filter with time (c).

(a)

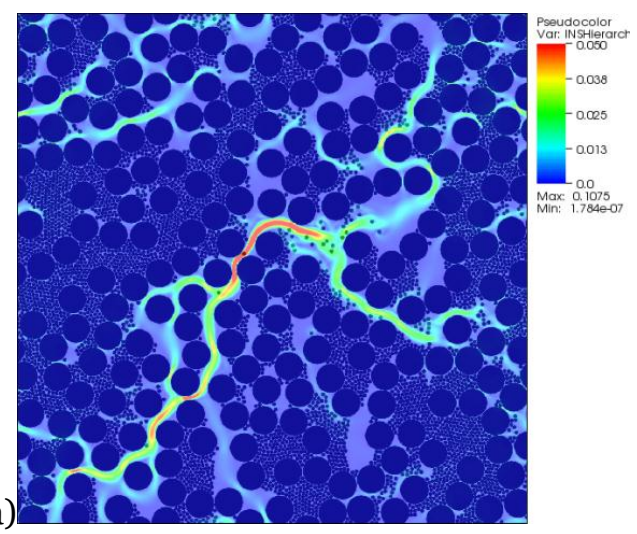

(b)

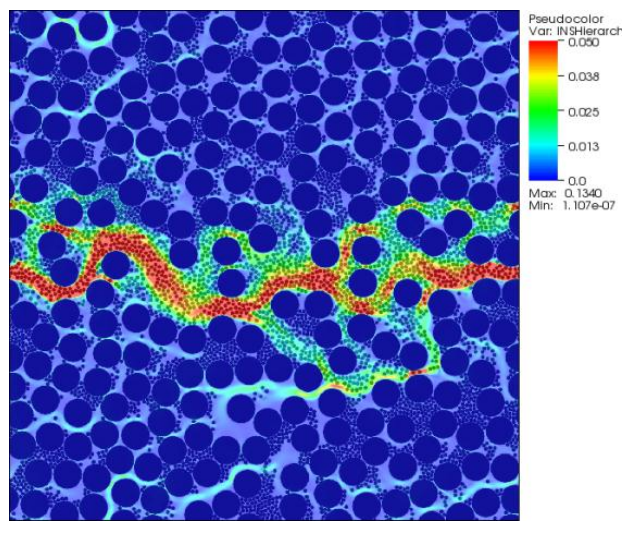

Figure 47. Soil migration through "damaged" filter. (a) case of effective filter with moderate damage. Soil particles effectively clog increased pore spaces. (b) case of non-effective filter with significant damage. Soil particles are washed out through the crack and are not retained by the filter. The color scale for velocity field is from 0 to $0.05 \mathrm{~m} / \mathrm{s}$. 


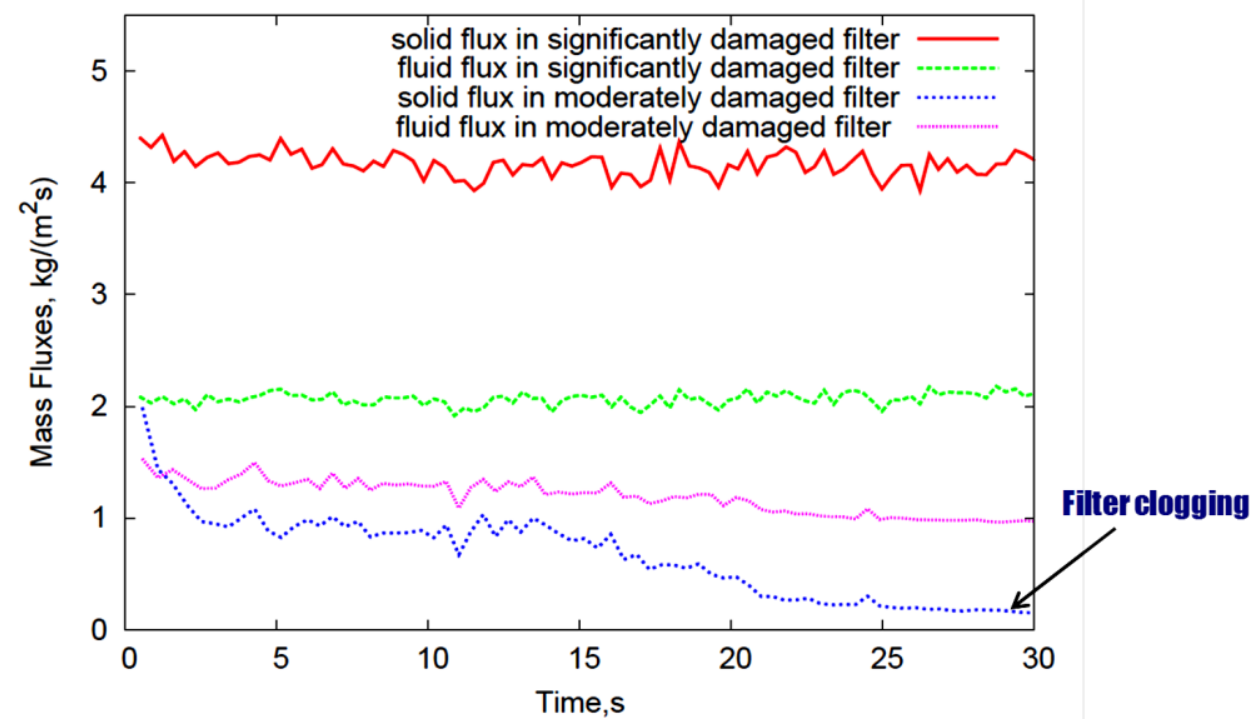

Figure 48. Comparison of soil and fluid fluxes in moderately damaged filter and significantly damaged filter.

\section{MESOSCALE SIMULATIONS}

Particulate flows occur in a wide range of industrial applications and in nature. Clearly, it is difficult to have one single simulation method that can cover all length and time scales. Currently, there is a hierarchy of methods that can cover different length and time scales with different levels of details (Zhu et al. 2007).

When the computational grid size is much larger than particle size usually a two-fluid (or multiphase) approach is used. The computational fluid dynamics two-fluid approach is often associated with methods described in Gidaspow (1994) and various implementations are available, such as the commercial code FLUENT (2001) and the DOE/NETL code MFIX (Syamlal et al. 1993). These two-fluid codes usually utilize a granular-kinetic-theory based constitutive model to represent the "fluid" comprised of fluidized particles, and empirical two-way coupling relations between fluid and particles. An advantage of the multi-fluid model is that in principle, it can be used to compute any multiphase flow regime. However, the effective use of these models strongly depends on the constitutive or closure relations for the solid phase and momentum exchange between phases. For two-phase systems comprised of billions of particles (like most fluidized-beds or pneumatic-transport systems for fine particulates), such continuum models are the only computationally viable simulation methods available. In fact, development of a general theory to correctly represent granular flow with fluid as a continuum is still a challenging research area.

If the cell size is larger than the particle size, a combined CFD-DEM coupling approach is used. In this approach, the motion of individual particles is obtained by solving Newton's equations of 
motion, while the flow of continuum gas is determined by the CFD on a computational cell scale. A variety of continuum fluid codes coupled with discrete-element method (DEM) particles, are utilized by several researchers ( Zhu et al., 2007). The algorithm relies on the parameterizations of drag terms similar to the Ergun equation (Ergun 1952) for static packed beds, or the Wen-Yu equation for moving beds (Wen and Yu 1966). While some questions regarding parameterizations of drag terms still remain open, this approach might be suitable for simulations of intermediate-scale system (about a million particles) and promises to be a powerful tool. However, there are some restrictions of the algorithm and the CFD-DEM coupling algorithm, since it assumes that the cell size in the CFD model should be larger than the particle size. This may result in using a fairly coarse mesh in the areas (nozzles, openings) where only few particles across an important geometric feature are considered as well as placing severe restrictions on the maximum particle size that can be included in simulations.

In the present work, the contact is detected in the region where two particles overlap based on the volume of fraction function and does not require expensive contact searching algorithms. While this approach would not be applicable for "classical" DEM algorithms, since the particle domain is not resolved in DEM, it is a natural fit for fluid-particle problems. Moreover, it is easily extendable to non-spherical objects, which are hard to accommodate in a typical DEM approach. In addition, if the application domain has complex geometry (e.g., in pneumatic conveying systems), it needs to be represented in the numerical code. This is done using the volume of fraction technique that allows different geometric objects of different shapes and sizes.

Parallel capabilities are important for improving efficiency of the numerical method. Despite the large number of publications devoted to the fictitious domain method, its parallel implementation is rarely discussed. A parallel implementation of the method is based on the Lawrence Livermore Structured Adaptive Mesh Refinement Application Infrastructure (SAMRAI) library and allows local grid refinement at the solid interfaces. Eulerian methods with AMR and advanced interface tracking capabilities are well suited for problems where accurate tracking of solid-fluid and fluid-fluid interfaces are required. The purpose of AMR is to concentrate the computational work near the regions of interest (e.g., solid boundary or maximum vorticity region). When properly designed, AMR can significantly reduce the computational effort required to obtain a desired level of accuracy in the simulation. Griffith et al. (Griffith et al. 2007, Griffith et al. 2010) extended SAMRAI infrastructure for fluid-structure interaction problems using immersed boundary method (Peskin 1977). This work extends this framework using Distributed Lagrance Multiplier technique to describe fluid-solid interactions for the case of a large number of rigid particles. The convergence properties and scalability of numerical algorithm are investigated for different benchmark examples and application. 
NUMERICAL MODEL

Governing equations and algorithm are presented below. The particle domain is denoted as $P(t)$, where $\partial P$ is the interface between the particle and the fluid. $F$ is the fluid domain that is not shared with the particle. The entire computational domain that includes both the fluid and the particles is denoted by $F \cup P$. The governing equations in the fluid domain can be written as:

$$
\begin{aligned}
& \rho\left(\frac{\partial \vec{u}}{\partial t}+(\vec{u} \cdot \nabla) \vec{u}\right)=\nabla \cdot \sigma+\rho g+\vec{f}, \text { in } F \cup P \\
& \nabla \cdot \vec{u}=0, \text { in } F \cup P \\
& D[\vec{u}]=0, \text { in } P(t) \\
& D[\vec{u}] \cdot \vec{n}=0, \text { on } \partial P(t) \\
& \vec{u}=\vec{u}_{s}, \text { in } P(t)
\end{aligned}
$$

where $\vec{u}$ is the fluid velocity, $p$ is the pressure, $\rho$ is the density that is equal to $\rho_{f}$ in the fluid domain and equal to $\rho_{s}$ in the particle domain, $g$ is gravity, $\vec{n}$ is a unit normal on the particle surface. The rigid body velocity inside the particle $\vec{u}_{S}$ is represented as:

$$
\vec{u}_{s}=\vec{U}+\vec{\Omega} \times \vec{r}
$$

where $\vec{U}$ and $\vec{\Omega}$ are the translational and angular velocities of the particle, respectively, and $\vec{r}$ is the position vector of the point with respect to the particle centroid. Force $\vec{f}$ that appears in the right hand side of the momentum equation is non-zero only in the particle domain and arises as a result of the rigid body motion constraint in the particle domain. $D[\vec{u}]$ is the deformation-rate tensor defined as: 


$$
D[\vec{u}]=\frac{1}{2}\left[\nabla \vec{u}+\nabla \vec{u}^{T}\right]
$$

The stress tensor is then given by:

$$
\sigma=-p I+\tau
$$

where $I$ is the identity tensor, $p$ is the pressure and $\tau$ is the viscous stress tensor given by:

$$
\tau=\mu D[\vec{u}]
$$

for a Newtonian fluid, where $\mu$ is the viscosity of the fluid. The particle domain in this formulation is treated as a fluid with an additional constraint (Lagrange multiplier) to impose the rigid body motions in such a way that deformation-rate tensor $D[\vec{u}]$ within the particle domain is zero. It should be noted that the representation (39) for the solid velocity is sufficient and necessary condition for the validity of (40). Stresses can develop in the rigid fluid domains, but the only displacements allowed are strain-free translations and rotations.

The integration of governing equations is done using fractional time stepping approach at time interval $\left[\mathrm{t}^{\mathrm{n}}, \mathrm{t}^{\mathrm{n}+1}\right]$. In the present algorithm, the velocity and pressure are cell-centered quantities. The velocity is defined at multiple integers of $\Delta t$, whereas the pressure is defined at half-time steps. The system of equations (34)-(36) - with boundary conditions (37)-(38) are solved numerically using operator-splitting technique that combines incompressibility condition, advection-diffusion as a first step; the constraint of rigid body motion in the particle domain and the related distributed Lagrange multiplier technique as the second step.

- First step: We solve the Navier-Stokes equations on the Eulerian grid. We neglect the presence of the particles at this point, except for their density. We project the particles onto the Eulerian fluid grid by computing a volume fraction of each particle, which ranges between 0 if the grid cell is completely outside any particles and 1 if it is completely inside:

$$
\rho \frac{\overrightarrow{\tilde{u}}-\vec{u}^{n}}{\Delta t}+\nabla p^{n+1 / 2}=R^{n+1 / 2}
$$




$$
\nabla \cdot \tilde{u}=0
$$

where $\mathrm{R}^{\mathrm{n}+1 / 2}$ represents all terms in the right-sides of momentum equations except the pressure gradient terms. An unsplit second order Godunov procedure is used to approximate the non-linear advection term that appears in the momentum equations using both velocities defined at the centers of the Cartesian grid as well as velocities defined at the cell faces. The density is computed as: $\rho=\rho_{s} \phi+\rho_{f}(1-\phi)$, where $\phi$ is the solid volume fraction which is equal to 1 in the solid domain and equal to 0 in the fluid domain.

- Second step: The code uses a Lagrange multiplier method to enforce the correct no-slip boundary condition over the surface of the particles and inside them. We enforce rigid body motion on the particles by taking the average translational and rotational velocities within each particle region, and setting it equal to the particle's Lagrangian velocity. The velocity at each Eulerian grid point is updated accordingly. The particle velocity in a given cell is split on translational $\vec{U}$ and rotational $\vec{\Omega}$ parts as:

$$
\vec{u}_{s}=\vec{U}+\vec{\Omega} \times \vec{r}
$$

where $\vec{r}$ is a vector which connects a particle centroid and a center of the considered grid cell. Particle velocities $\vec{U}$ and $\vec{\Omega}$ are computed by integration of the provisional velocity field $\overrightarrow{\tilde{u}}$ in the solid domain as:

$$
\begin{aligned}
& M \vec{U}=\int_{P} \rho_{s} \tilde{u} d V \\
& I_{p} \vec{\Omega}=\int_{P} r \times \rho_{s} \tilde{u} d V
\end{aligned}
$$

where $M$ is the mass of a particle and $I_{p}$ is the moment of inertia of a particle. The velocity field $\vec{u}^{n+1}$ is then updated in the solid, fluid and mixed domains (where both solid and fluid are presented) as: 


$$
\begin{cases}\vec{u}^{n+1}=\vec{u}_{s}, & \text { in solid cells } \\ \vec{u}^{n+1}=\overrightarrow{\vec{u}}, & \text { in fluid cells } \\ \vec{u}^{n+1}=\frac{\rho_{s} \phi \vec{u}_{s}+\rho_{f}(1-\phi) \overrightarrow{\tilde{u}}}{\rho}, & \text { in mixedcells }\end{cases}
$$

where $\phi$ is the solid volume of fraction, computed based on the particle position at time $\mathrm{n}$. This step could be considered as finding force $\vec{f}$ that modifies provisional velocity field $\overrightarrow{\tilde{u}}$ such that the final velocity field $\vec{u}^{n+1}$ satisfies rigid body motion constraints. Therefore the force $\vec{f}$ can be defined as:

$$
\vec{u}^{n+1}=\overrightarrow{\tilde{u}}+\frac{\vec{f} \Delta t}{\rho}
$$

Also as it was mentioned before the final velocity should provide zero deformation-rate tensor as:

$$
D\left[\vec{u}^{n+1}\right]=0
$$

Since in the method considered here the final velocity in the solid domain is known at this step and it is equal to $\vec{u}_{s}$, condition (50) is satisfied automatically and the final velocity is calculated from Eq. (48) directly. This approach is similar to one implemented in Patankar (2001).

- Third step: If at this point, the volume fraction in a grid-cell is greater than one, a collision has occurred. We use a visco-elastic collision model to apply appropriate forces to each particle involved in a collision, and then update their velocities appropriately. If the particle Reynolds number and solid volume fraction are low, particles do not interact and $U^{n+1}$ and $X^{n+1}$ defined at the previous step are the final velocities that describe velocity field inside the particle. When the concentration of particles is high enough they begin to interact with each other. If the viscosity of fluid is high it would prevent particles from colliding with each other. But if fluid forces are insufficient to prevent particle contacts, the separately applied collision forces $\vec{F}^{n+1}$ similar to DEM models (Cundall and Strack, 1979) are used in our code. In the present work the contact 
is detected in the region where two particles overlap based on the volume of fraction function $\phi$. The condition $\phi>1$ means that more than one particle exists in the given grid cell, therefore additional collision force is applied in each grid cell where particles overlap. The collision force acts along the normal ( $\vec{F}_{n}$-component) as well as the tangential direction ( $\vec{F}_{t}$-component) at the point of contact between two particles. For spherical particles, the normal and tangential directions are defined by the line joining the centers of two colliding particles and two lines perpendicular to it, respectively. The components of the normal collisional force is computed in each cell where particles overlap and then integrated over the overlapped region as:

$$
\vec{F}_{n}=-\sum_{i j k}\left(k_{n} V_{i j k}+d_{n} V_{i j k} v_{n}\right) / m_{p}
$$

where $V_{i j k}$ - volume of cell $i j k$ in the overlapped region, $v_{n}$ - relative velocity between the two interacting particles in the normal direction, $k_{n}$ - normal spring constant (or stiffness), $d_{n}$ - damping coefficient in the normal direction, $m_{p}$ - particle mass. The tangential component is computed according to a Coulomb friction law as:

$$
\vec{F}_{t}= \begin{cases}\sum_{i j k} d_{t} V_{i j k} \vec{v}_{t}, & \left|F_{t}\right| \leq \mu_{f}|F n| \\ -\mu_{f}|F n| \vec{t}, & \left|F_{t}\right| \geq \mu_{f}|F n|\end{cases}
$$

where $\vec{v}_{t}$ - relative velocity between the two interacting particles in the tangential direction $\vec{t}, \mu_{f}$ is the friction coefficient, and $d_{t}$ is the damping coefficient in the tangential direction. Geometrically, the collision force depends on the overlap volume between two particles. In the case of particle-wall collisions the computational procedure is trivially extended, taking into account that velocity of walls is zero. In general case the stiffness and damping coefficients for particle-wall and particle-particle interactions are different.

Once collision forces are calculated, the final particle velocities (translational and rotational) are updated using velocity field from previous time step as: 


$$
\begin{aligned}
& \vec{U}^{n+1}=\overrightarrow{\widetilde{U}}^{n+1}+\vec{F}^{n+1 / 2} \Delta t \\
& I_{p} \vec{\Omega}^{n+1}=I_{p} \overrightarrow{\widetilde{\Omega}}^{n+1}+\left[\vec{r} \times \vec{F}^{n+1 / 2}\right] \Delta t
\end{aligned}
$$

where $\vec{U}^{n+1}$ and $\vec{\Omega}^{n+1}$ are particle velocities computed at the previous stage.

- Fourth step: Finally, we update the Lagrangian position of each particle and begin again for the next time step. Particle position $\vec{X}^{n+1}$ are explicitly updated by the following procedure:

$$
\vec{X}^{n+1}=\vec{X}^{n}+\frac{\vec{U}^{n+1}+\vec{U}^{n}}{2} \Delta t
$$

where $\vec{X}$ is the position of the particle centroid. If a particle or a solid body is represented as polyhedron with vertices coordinates $\vec{X}_{v}{ }^{n+1}$, their positions are updated as:

$$
\vec{X}_{v}^{n+1}=\vec{X}^{n+1}+[\vec{\Omega} \times \vec{r}]
$$

Once the vertices positions are defined the volume fractions for solid bodies are computed and used for computations at the next time step. The important advantage of the present collision method is that it is flexible enough to handle arbitrary particle shapes and size distributions and does not require extra parameterizations for fluid particle interactions. The shear friction forces discussed above can allow only slow movement in the tangent direction, not stop or reverse it. These results are inadequate for applications that require truly static friction, such as heap formation or angles of repose. In such situation, there is a threshold force below which the grains do not move at all, opposed by static friction. However, implementation of even a simple historydependent threshold rule is algorithmically complicated and will be a subject of the current work. Thus the applications in this report are limited by the visco-elastic collisional model described above. 


\section{CODE VALIDATION AND APPLICATION}

The determination of pressure drop through a packed bed as a function of fluid flow rate, geometrical constrains of the bed and physical properties of bed material is very critical, for example, in hydraulic and pneumatic devices. The well-known empirically derived equation used for that purpose has been proposed by Ergun (1952) based on experimental measurements:

$$
\frac{\Delta p}{L}=A \frac{(1-\varepsilon)^{2}}{\varepsilon^{3}} \frac{v}{D_{p}^{2}} u+B \frac{(1-\varepsilon)}{\varepsilon^{3}} \frac{\rho_{f}}{D_{p}} u^{2}
$$

where $D_{p}$ is the pressure drop through the packed bed, $L$ is the bed length or height, $\varepsilon=V_{f} / V$ is the bed porosity, where $V_{f}$ is the voids volume, $V$ is the total volume, $u$ is the superficial velocity at the exit of bed, $v$ is the fluid kinematic viscosity, and $D_{p}$ is the particle diameter. Ergun Eq. (57) incorporates momentum loses due to viscose effects (first term in (57) which is important in laminar regime) and inertia effects (the second term in (57) which dominates in the turbulent regime). Ergun equations are often used in more general form by introducing a friction coefficient $K$ which is defined as

$$
K=A+B \operatorname{Re}^{c}
$$

A standard form of Ergun equation uses the values of empirical constants $A=150, B=1.75, C=$ 1. However many other studies have been performed and published to check the appropriate choice of the empirical constants $A, B$ and $C$ in Equation (58). Some authors proposed, values in the range 150-200 for $A$ and 1.7-4.0 for $B$ as well as functional forms for these coefficients that depends on both porosity and Reynolds number $\operatorname{Re}=D_{p} / v(1-\varepsilon)$, see overview in Plessis (2001). Vortwek and Brunn (1994) proposed to use values $A=181, B=2.01$ and $C=0.96$ to estimate the pressure drop in randomly packed beds. Franzen (1979) found that for $A=164.97$, $B=1.976$ and $C=0.9$, (it will be nice to have the same significant digit, it is inconsistent throughout the document) Equation (57) gives good estimate of the pressure drop for cubic packing of spheres. The reason for the variation in the constants was determined as the variations in particle geometry and orientation, as well as macroscopic properties of the packing. It should be noted that Ergun equation was derived for densely packed beds, and is not expected to be valid for high void ratios. For that range (when porosity is smaller than 40\%), normally the Wen and Yu equation (Wen and Yu 1966) is used. However this equation 
significantly under predicts the drag force at higher Reynolds numbers (Beetsra et al. 2007). Also the transition from Ergun to Wen and Yu equation is not a smooth function. For moving particles the situation becomes even more complicated since particles begin to interact with each other and may dissipate additional energy that affects the pressure drop. Therefore detailed mesoscale simulations that resolve the flow around each particle are needed to predict flow characteristics such as the pressure drop and volumetric flow rates in these systems.

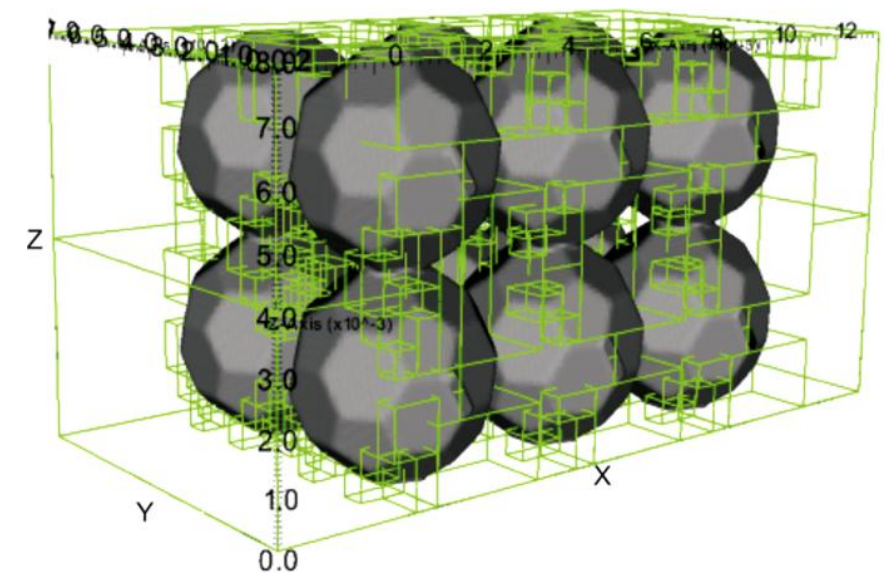

Figure 49. Computational domain and geometry of AMR patches for simulation of fluid flow through the cubic array of spheres.

As the first step, we validate our code against experimental and empirical data for both randomly and regularly arranged packing in different flow regimes and Reynolds numbers. First we consider configuration that consists of the 12 monodisperse spheres of radius $R=0.002 \mathrm{~m}$ arranged in the cubic packing (Figure 49). Domain size is $0.012 \mathrm{~m} \times 0.008 \mathrm{~m} \times 0.008 \mathrm{~m}$. We consider two flow regimes with Reynolds numbers $\operatorname{Re}=18$ and $\operatorname{Re}=900$. A constant inflow velocity field of $u=0.02 \mathrm{~m} / \mathrm{s}$ was prescribed at the left boundary and directed along $x$-axis. Open boundary condition is set at the right side. Other boundaries are chosen to be periodic. Cells with the high vorticity magnitude are tagged for adaptive mesh refinement. An adaptive computation employs a total of two levels with refinement ratio of 2 .

Four different grids (i) $36 \times 24 \times 24$, (ii) $72 \times 48 \times 48$, (iii) $144 \times 96 \times 96$ and (iiii) $288 \times 192 \times 192$ for the finest level were chosen. The code employs adaptive time step with Courant number 0.85. It takes about $1.5 \mathrm{~h}$ of wall clock time to reach steady state (about 4000 timesteps) for configuration with resolution (iii) 144 x 96 x 96 on 16 nodes Opteron Linux cluster. The porosity is $\varepsilon=47.64 \%$. Figure 50 shows values of friction coefficient $\mathrm{K}$ in different data sets that include mentioned earlier Ergun (1952), Franzen (1979), Hovekamp (2002), Martin et al. (1951), Vortwek and Brown (1994). For small Re numbers, difference in the data sets for both random and cubic packing is small and our simulations agree well with existing data. For high $\operatorname{Re}$ numbers, there is a difference of about $40 \%$ for the friction coefficient in existing empirical and 
experimental data (Figure 50). We found that our computed results lie within the range of available experimental data and agree well with the experimental data of Martin et al. (1951). Another validation of the code for high Reynolds numbers will be presented in this section for periodic array of spheres.
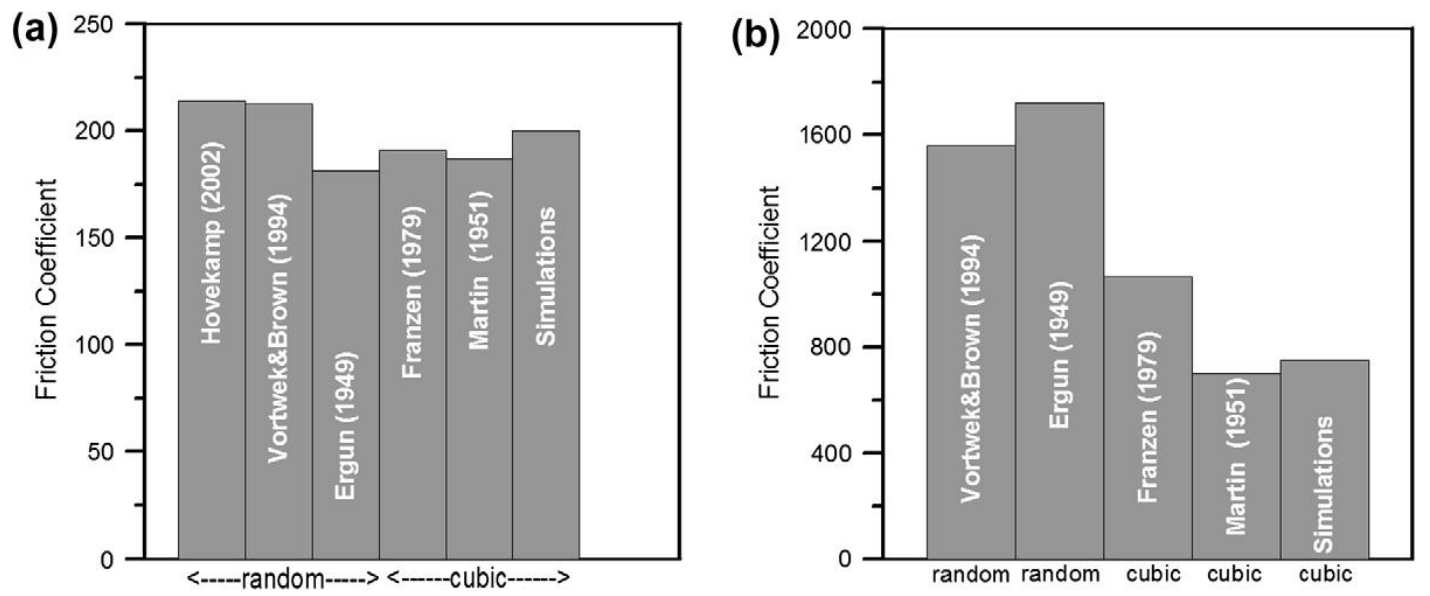

Figure 50. Comparison of the computed friction coefficient with available data for random and cubic packing $s$ at $\operatorname{Re}=18$ (left panel) and $\operatorname{Re}=900$ (right panel). Data from Hovekamp (2002), Vortwek and Brunn (1994), Ergun (1952), Franzen (1979), and Martin et al. (1951) are shown.
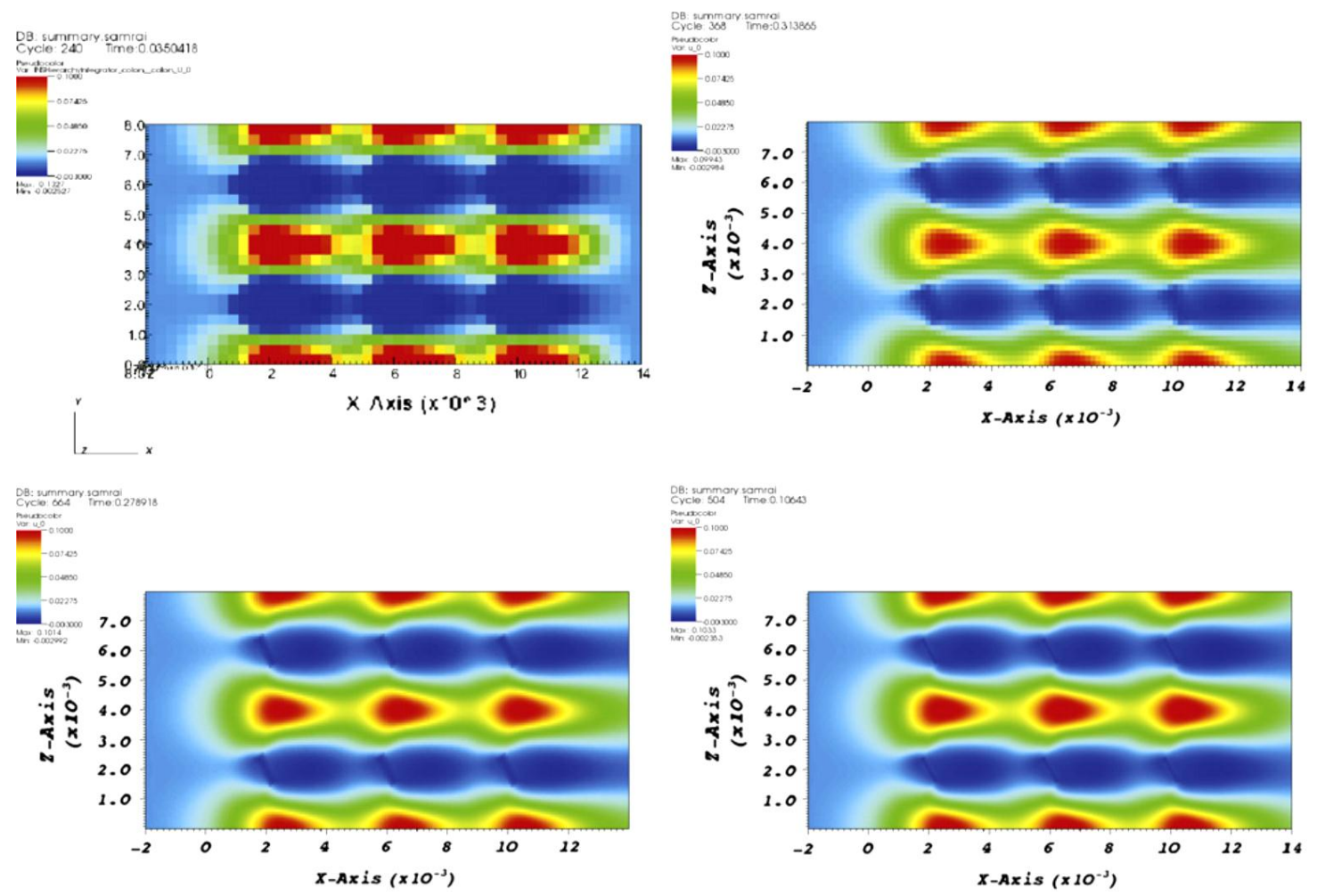

Figure 51. Horizontal velocities (in $\mathrm{m} / \mathrm{s}$ ) at the central slice ( $\mathrm{x}-\mathrm{z}$ plane at $\mathrm{y}=\mathbf{0 . 0 0 4} \mathrm{m}$ ) for the cubic packing of spheres at $\operatorname{Re}=18$. 


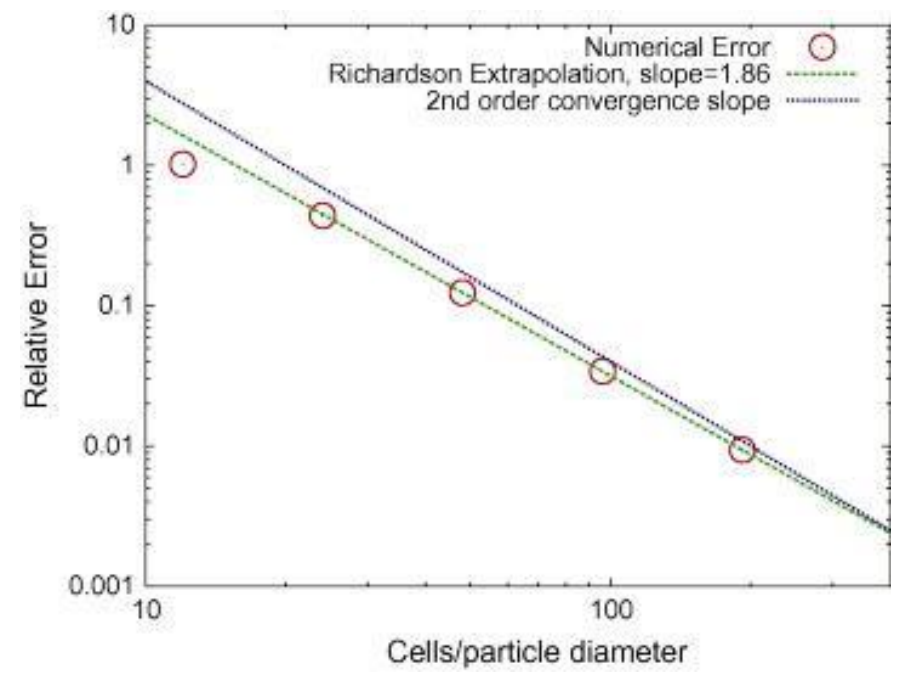

Figure 52. Convergence rate for $\mathrm{Re}=18$. Numerical error versus resolution is shown.

We use Richardson extrapolation $f(h)=f_{\text {exact }}+C h^{p}$ to estimate the convergence order using computed values of $f(h)$. Here $f$ is a calculated parameter (pressure drop in our case), $h$ is some measure of grid spacing, $C$ is a constant, and $p$ is the order of convergence. We calculate the relative error $(\mathrm{RE})$ of our computed solution $\mathrm{f}$, with respect to the exact solution $f_{\text {exact }}$, where $R E=\left\|f-f_{\text {exact }}\right\| /\left\|f_{\text {exact }}\right\|$. Figure 52 and Figure 54 present relative errors for different resolutions. Based on the results of simulations we found that the convergence rate is close to the second-order: 1.86 for $\mathrm{Re}=18$ and 1.8 for $\mathrm{Re}=900$. However the low Reynolds number flow requires less resolution to get converged solution then the high Reynolds number flow. About 24 cells per particle are needed to describe flow behavior for $\operatorname{Re}=18$. In the case of $\operatorname{Re}=$ 900 , the resolution needs to be twice higher to get the same order of error. This is mainly because of the flow separation and turbulent boundary effects that require finer grid resolutions to describe them adequately (Figure 51 and Figure 53).

In these simulations we do not use any turbulent model. However in future studies we may need to incorporate additional drag terms to be able to simulate high Re number flow regimes with relatively modest resolution (e.g. the same resolution as for small Re numbers). It should be also mentioned, that the grid resolution needed for converged solution depends on particular configuration and porosity. For densely packed beds, the flow characteristics are constrained by the maximum resolution available in the void space between particles. Fineresolution simulations are required to describe flow effects through these small void spaces for high Re numbers. Therefore an AMR technique that allows increasing resolution in fluid domain would be very useful. 

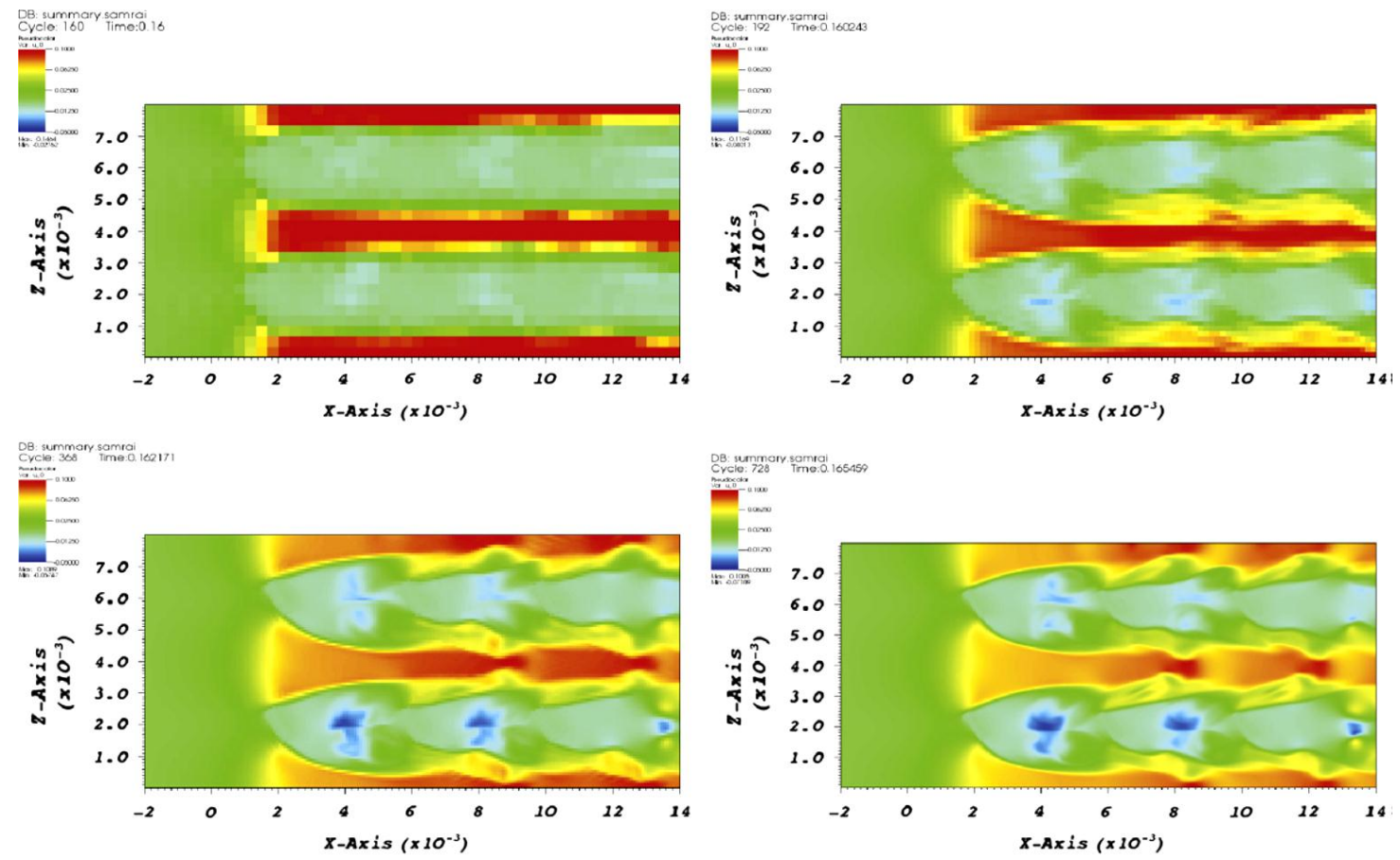

Figure 53. Horizontal velocities (in $\mathrm{m} / \mathrm{s}$ ) at the central slice ( $x-z$ plane at $y=0.004 \mathrm{~m}$ ) for the cubic packing of spheres at $\operatorname{Re}=900$.

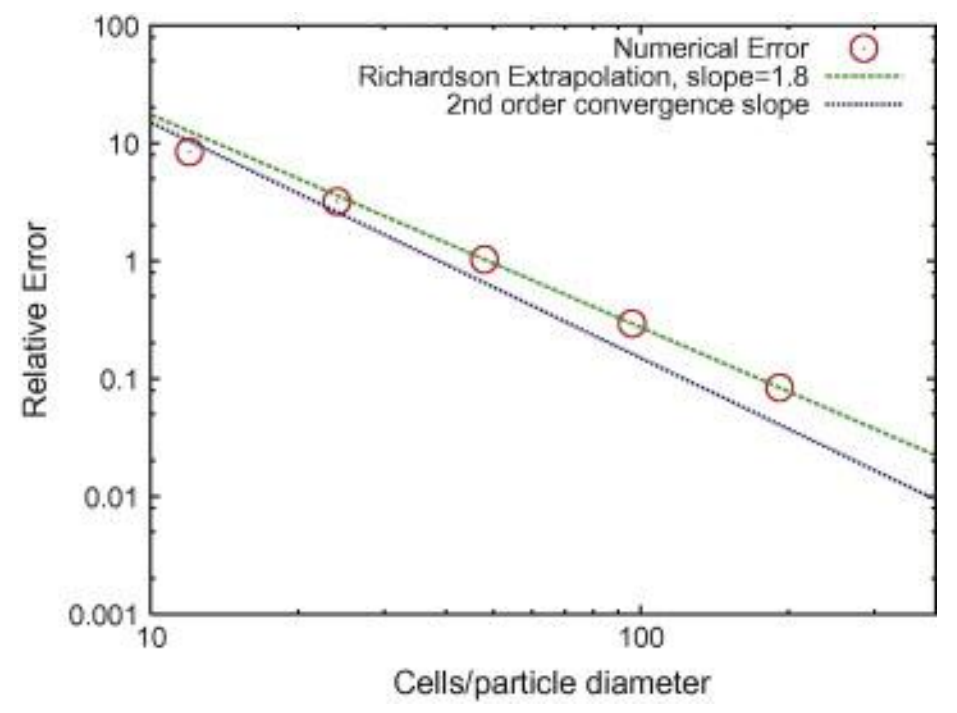

Figure 54. Convergence rate for $\operatorname{Re}=900$. Numerical error versus resolution is shown.

The next example is flow in a periodic domain of randomly packed spheres of radius $\mathrm{R}=$ $0.11283 \mathrm{~m}$. Re number in this configuration is about 900 and the solid packing ratio is $60 \%$. $\mathrm{A}$ superficial velocity is specified as $u=0.008 \mathrm{~m} / \mathrm{s}$. The periodic random distribution of spheres is generated using Donev et al. (2005) algorithm. The pressure drop through the packed bed is illustrated in Figure 55. We perform a numerical analysis to estimate the convergence rate of numerical solution. The error in the pressure drop decreases with approximately second-order 
when refining the mesh (Figure 56). The difference for converged solution with Ergun equation (57) for coefficients $A=150$ and $B=1.75$ is found to be $7 \%$. This is quite reasonable agreement since the considered configuration with given porosity of $40 \%$ is a good representation of the cases investigated in the experiments by Ergun (1952). Our goal here is to present both code validations as well as provide guidance for the future code's applications. Therefore, in the next section we show how the local inhomogeneities in the local porosity distribution of randomly packed bed with the same global parameters (porosity, particle size) may influence the flow and transport properties.

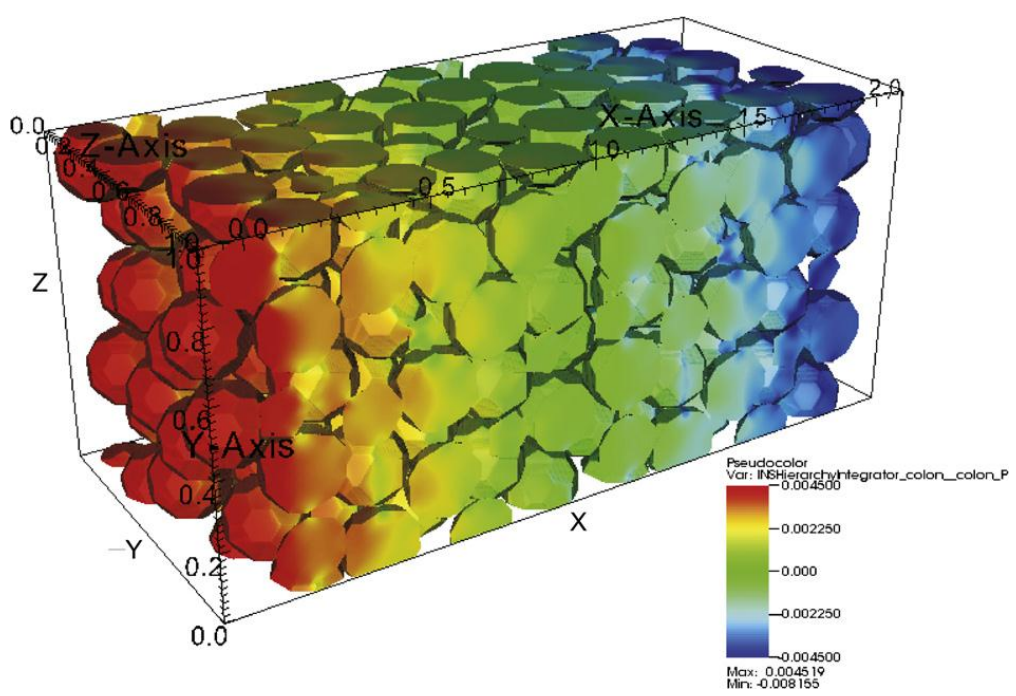

Figure 55. Pressure drop (in $\mathrm{Pa}$ ) through the randomly packed bed.

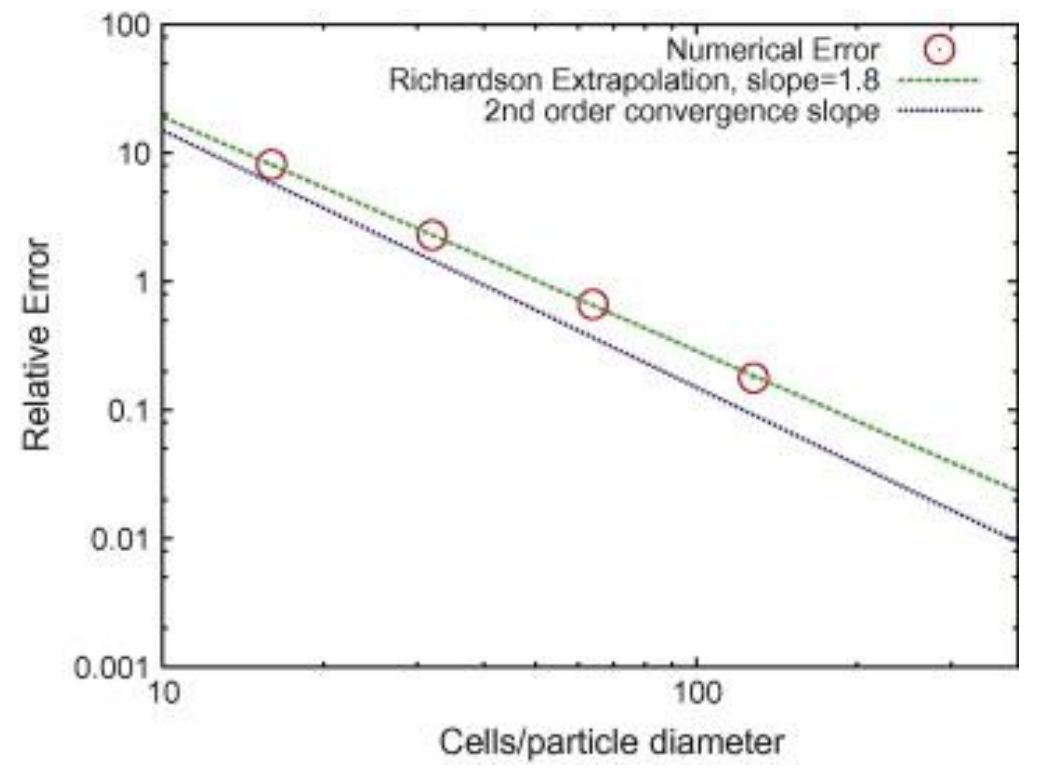

Figure 56. Convergence rate. Numerical error versus resolution is shown. 

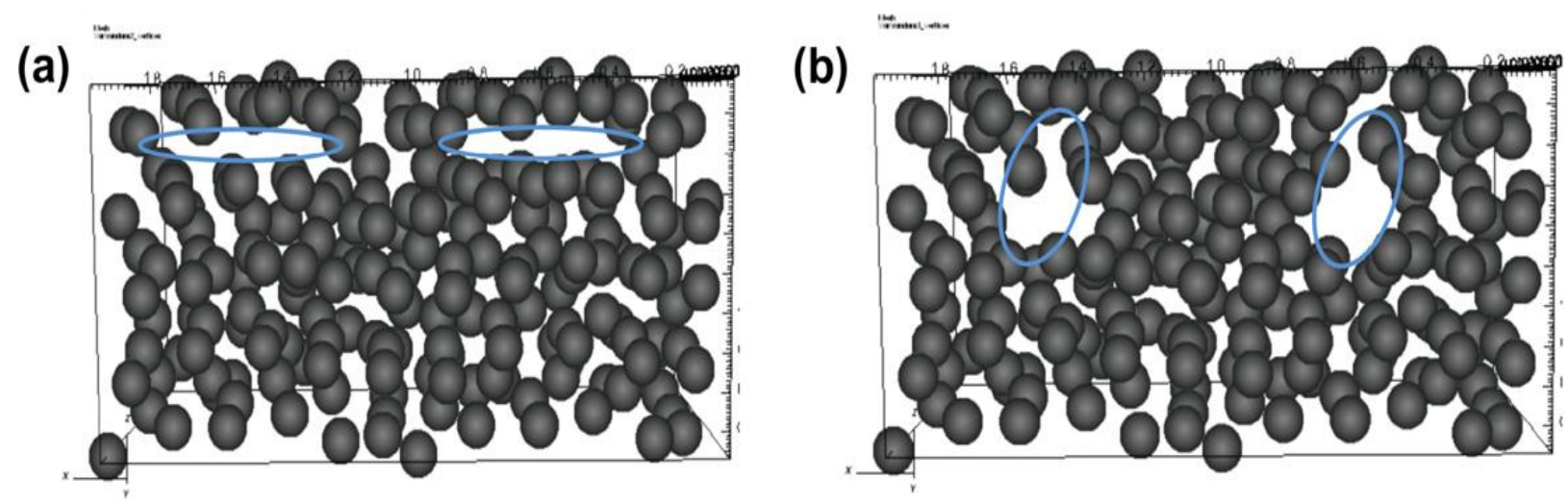

Figure 57. Two different packings with the same porosity of $42 \%$. In configuration 1 (a) the anisotropy in the vertical $x-y$ plane is introduced by removing few particles from the initial randomly packed structure with porosity $36 \%$ at $z=0.8 \mathrm{~m}$. In configuration 2 (b) the anisotropy in the horizontal $y_{-} z$ plane is introduced in the same manner at $x=0.3 \mathrm{~m}$.

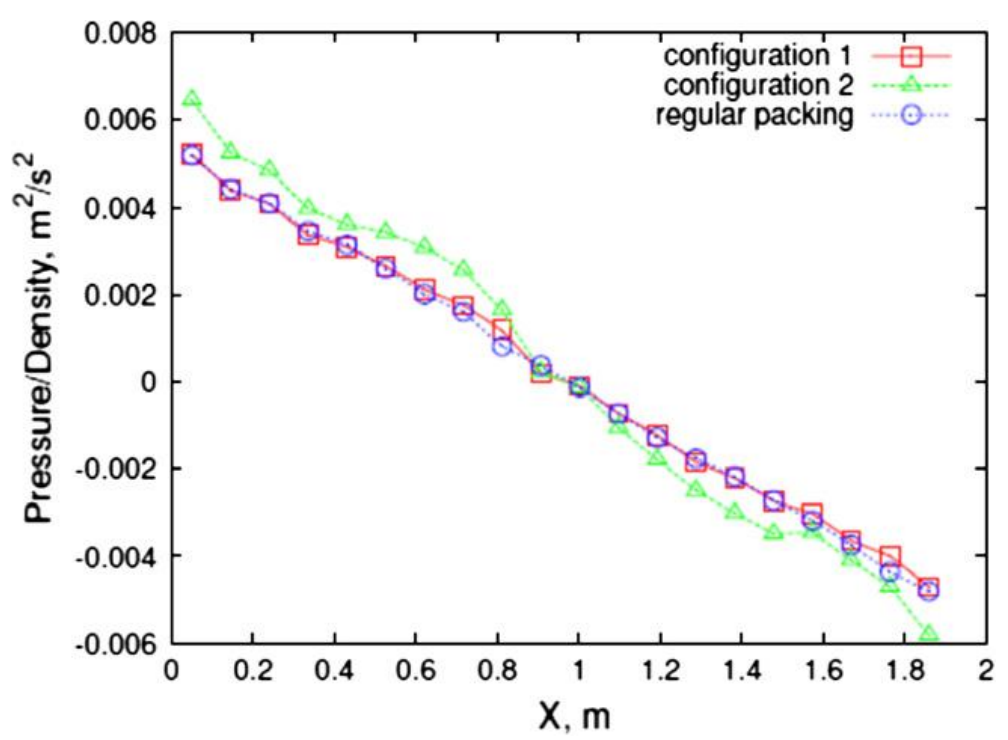

Figure 58. Comparison of the pressure drop in configuration 1, configuration 2 and regular random packing with porosity $42 \%$.

It is previously mentioned that Ergun equation (57) is widely used as a drag parameterization in the coarse grid models. But it includes only averaged properties of the packed bed such as porosity. Thus, pressure drop correlations, using only the mean porosity as system describing parameter, can give an approximate range of the pressure drop in packed beds, but do not account for the influence of the local structure on the global pressure drop and permeability. However, in many applications it is important to know how the local anisotropic inhomogeneities in the packing could affect averaged properties of the packed bed and flow characteristics. These local inhomogeneities in the granular medium can appear, for example, during dynamic loading as a result of homogeneous deformation with associative dilatancy or 
localized shear bands with even higher void ratios. Anisotropy can be observed also in the improperly fixed packings leading to the formation of bypass channels. We generate two different configurations of randomly packed spheres with the same porosity of $42 \%$.

In the first configuration (configuration 1 ) the anisotropy in packing in vertical $x-y$ plane is introduced, by removing few particles from initial randomly packed structure with porosity 36 $\%$ (Figure $57 \mathrm{a}$ ) at $\mathrm{z}=0.8 \mathrm{~m}$. The second configuration (configuration 2 ) is produced in the similar way by removing few particles in the horizontal plane $y-z$ at $x=0.3 \mathrm{~m}$ and $x=1.3 \mathrm{~m}$ (Figure $57 b)$. We estimate the pressure drop in both anisotropic configurations and compare it with the pressure drop in the regular random packing with the same porosity of $42 \%$. Figure 58 shows the computed pressure drop in each configuration. The pressure drop integrated in each crosssection along $\mathrm{x}$-axis is defined as $P(x)=\sum p(x, y, z) \Delta y \Delta z$, where $p(x, y, z)$ is a pressure value at point $(x, y, z)$. It is found the pressure drop in the configuration 1 is almost identical to the pressure drop in the regular packing with the same porosity of $36 \%$. The maximum difference in the pressure drop $\Delta P(x)$ between these two cases is found to be $22 \%$ (Figure 58).

Based on the averaged packing densities $\varepsilon_{1}(z)$ and $\varepsilon_{2}(x)$ in the granular bed (Figure 59) computed as $\varepsilon_{1}(z)=\sum \varepsilon(x, y, z) \Delta x \Delta y$ for configuration 1 , and $\varepsilon_{2}(x)=\sum \varepsilon(x, y, z) \Delta y \Delta z$ for configuration 2, we represent the packed bed as a combined medium that consists of two layers of granular material with different porosities and volumes (Figure 60).
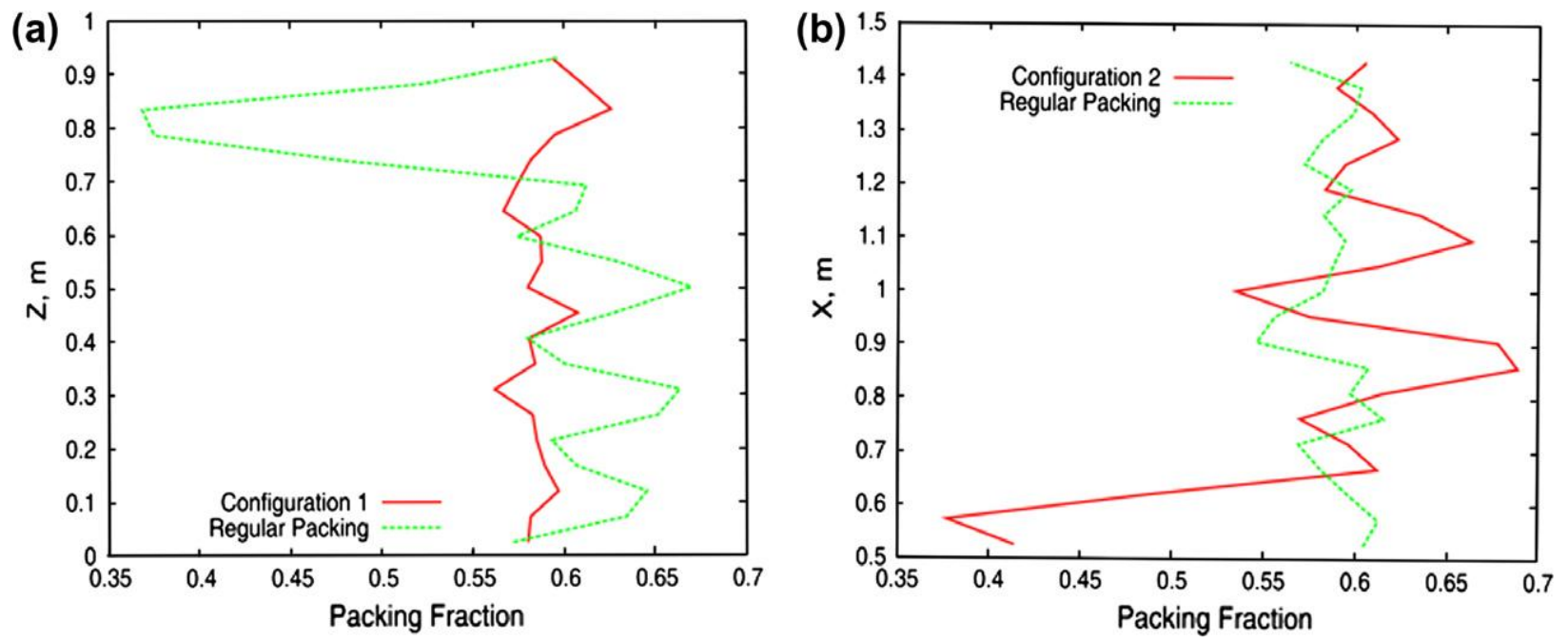

Figure 59. Averaged packing density for configuration 1 (a) and configuration 2 (b).

The first layer has width $0.7 \mathrm{~m}$ and porosity $36 \%$ and the second layer has width $0.3 \mathrm{~m}$ and porosity 56\%. For configuration 1 these layers are connected in parallel, whereas in configuration 2 they are connected in series. Analytical estimations from Ergun equation give us normalized pressure drop $0.0079 \mathrm{~m}^{2} / \mathrm{s}^{2}$ for configuration 1 , about $0.0118 \mathrm{~m}^{2} / \mathrm{s}^{2}$ for configuration 
2, and $0.0089 \mathrm{~m}^{2} / \mathrm{s}^{2}$ for the regular packing with porosity $42 \%$. Therefore the analytical estimation from Ergun equation predicts that the pressure drop in configuration 1 decreases by $11 \%$ and increases by $32 \%$ for configuration 2 from the pressure drop estimated for regular packing. The difference between directly calculated results and analytical estimations from Ergun equation can be explained by non-linear effects in the flow behavior at the grain scale (since introduced anisotropy was caused by inhomogeneity at the grain scale). This highlights importance of the local effects in packing on the global characteristics of the packed bed.
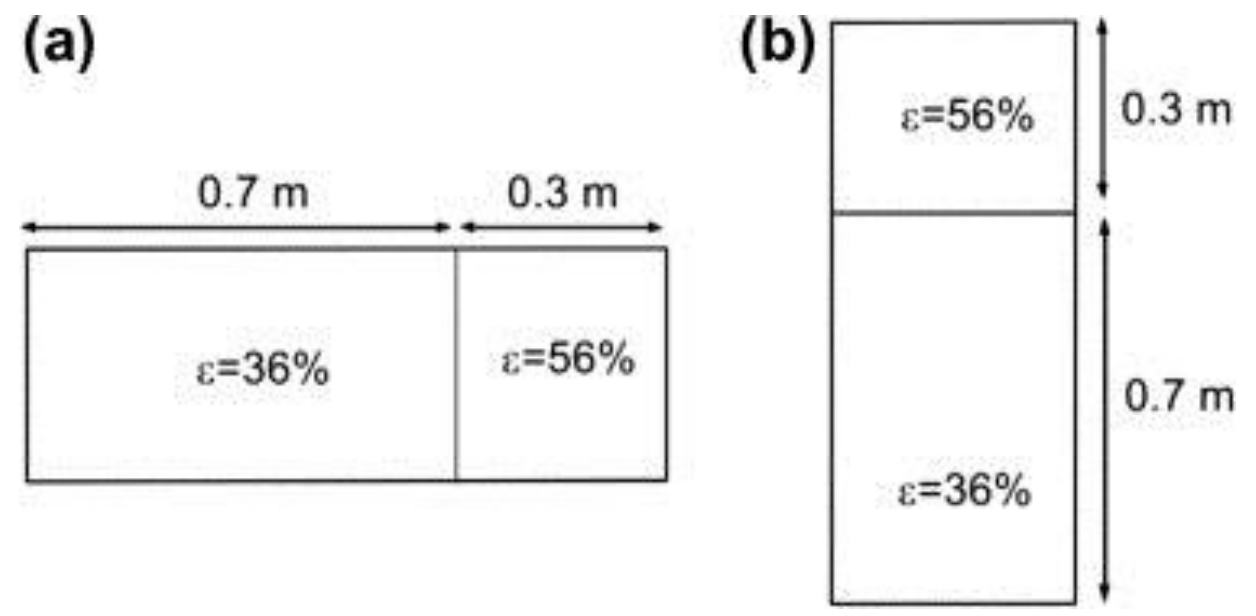

Figure 60. Schematic representation of two layers with different porosity and volume to estimate averaged properties of the combined medium.

We further verify our code by investigating the sedimentation of a cylindrical (2D) and spherical (3D) particle in a Newtonian fluid. We consider sedimentation of a particle with diameter $\mathrm{d}=$ $0.25 \mathrm{~cm}$ in a channel $0.02 \mathrm{~m} \times 0.06 \mathrm{~m}$ in $2 \mathrm{D}$ and $0.02 \mathrm{~m} \times 0.02 \mathrm{~m} \times 0.06 \mathrm{~m}$ in 3D. The fluid density is $\rho_{f}=1000 \mathrm{~kg} / \mathrm{m}^{3}$ and the particle density is $\rho_{s}=1250 \mathrm{~kg} / \mathrm{m}^{3}$.

The various Reynolds number dependent flow regimes are obtained by varying the fluid dynamic viscosity $\mathrm{m}$. We consider three cases here with $v=0.1,0.01$ and $0.0006 \mathrm{~Pa}$ s. Gravity acceleration $9.8 \mathrm{~m} / \mathrm{s} 2$ acts in a negative vertical direction. The simulation is started at $\mathrm{t}=0 \mathrm{~s}$ by dropping a particle at the center of the channel at $0.04 \mathrm{~m}$ depth. Different grid resolutions are considered ranging from 4 up to 128 grid cells per particle diameter. We choose this example since it is widely used as a benchmark example for testing numerical models, e.g. fictitious domain methods (Glowinski et al. 2001, Blasco et al. 2009) and immersed finite element method (Lee et al. 2009) with which we compare our numerical simulations. Figure 61 shows the vertical velocity and pressure profiles of the particulate flow with a single disk falling.

We compared computed numerical results for terminal velocity and particle position with other existing implementations and the result of comparisons is presented in Figure 59Figure 60. For the small Re number the flow is laminar and the disk reaches its terminal velocity relatively 
quickly (Figure 62). The maximum difference with existing numerical solution of Glowinski et al. (2001) for resolution $256 \times 768$ is about $2.4 \%$. The difference with other solutions is even less.

(a)

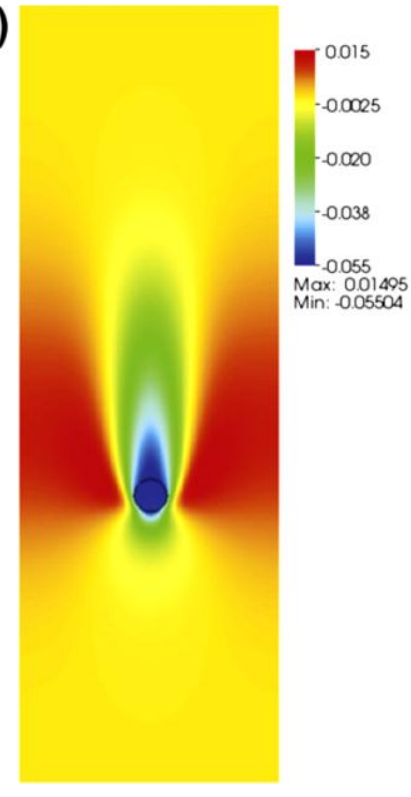

(b)

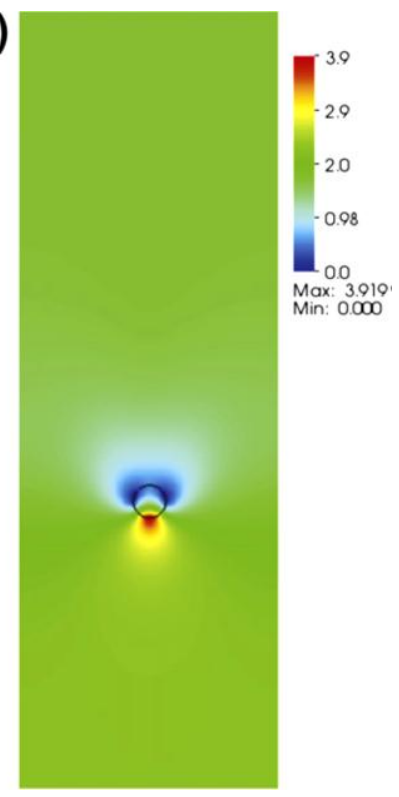

Figure 61. Numerical simulation of falling particle in a Newtonian fluid. A vertical velocity (in $\mathrm{m} / \mathrm{s}$ ) and pressure distribution (in $\mathrm{Pa}$ ) are shown.

In the case of sedimentation of spherical particle for small Reynolds numbers $\operatorname{Re}=\frac{w_{t} d}{v}$, where $w_{t}$ is a terminal velocity, there is an analytical solution for wt described by Stokes velocity (Stokes 1966, Lamb 1945) as

$$
w_{t}=\frac{\left(\rho_{f}-\rho_{s}\right) g d^{2}}{18 v}
$$

Heywood (1948) developed an approximate method for calculating the terminal velocity for wide range of Reynolds numbers. We compared computed settling velocities for falling spheres in three different regimes with Stokes and Heywood velocities for small Re numbers (for $\mathrm{Re}=$ $0.2, \operatorname{Re}=11$ ) and Heywood velocities for high Re numbers (for $\operatorname{Re}=500$ ). The overall agreement with existing theoretical values is good for both small and high Reynolds numbers (Figure 62). We also investigate the convergence rate for different Re numbers (Figure 62). The results agree well with the previous finding for the fixed array of spheres considered. Whereas for small Re numbers, only few cells per particle diameter (around 8) are required to get converged solution, for high Re numbers, about 32 grid cells are needed to represent the flow around particle and drag effects correctly. 

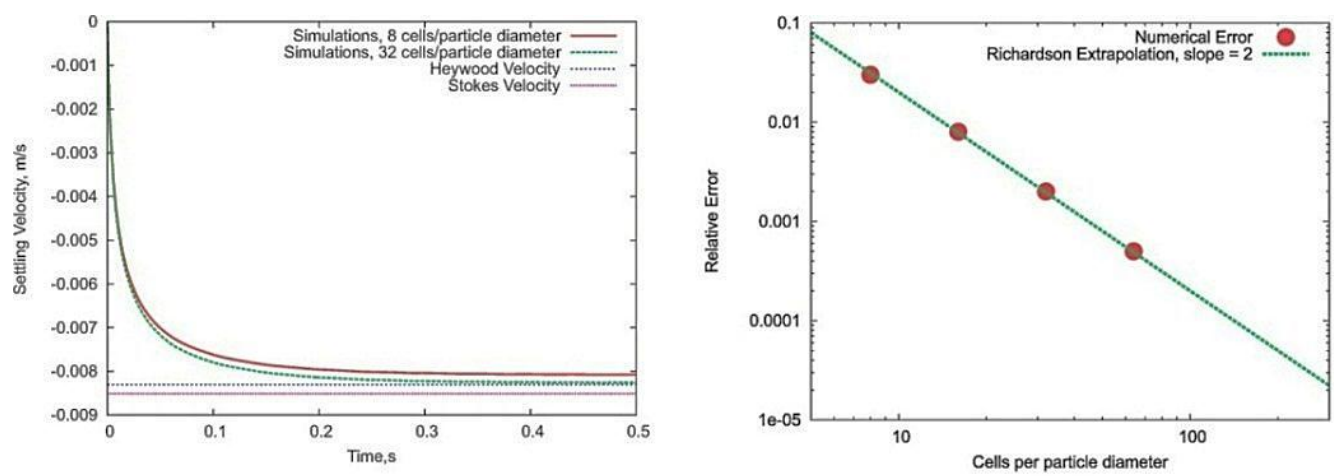

(a) $\mathrm{Re}=0.2$
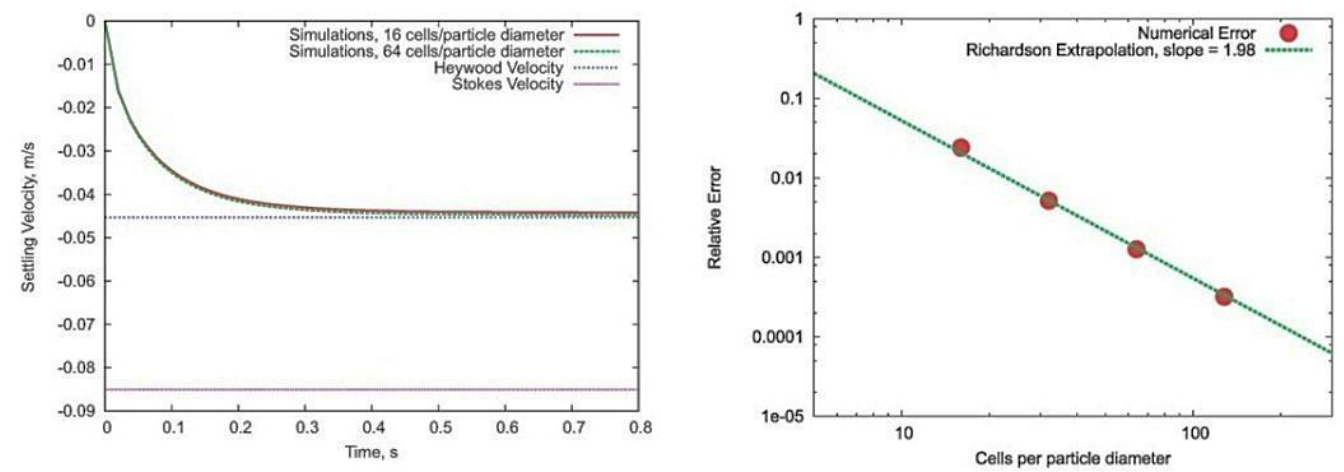

(b) $\operatorname{Re}=11$
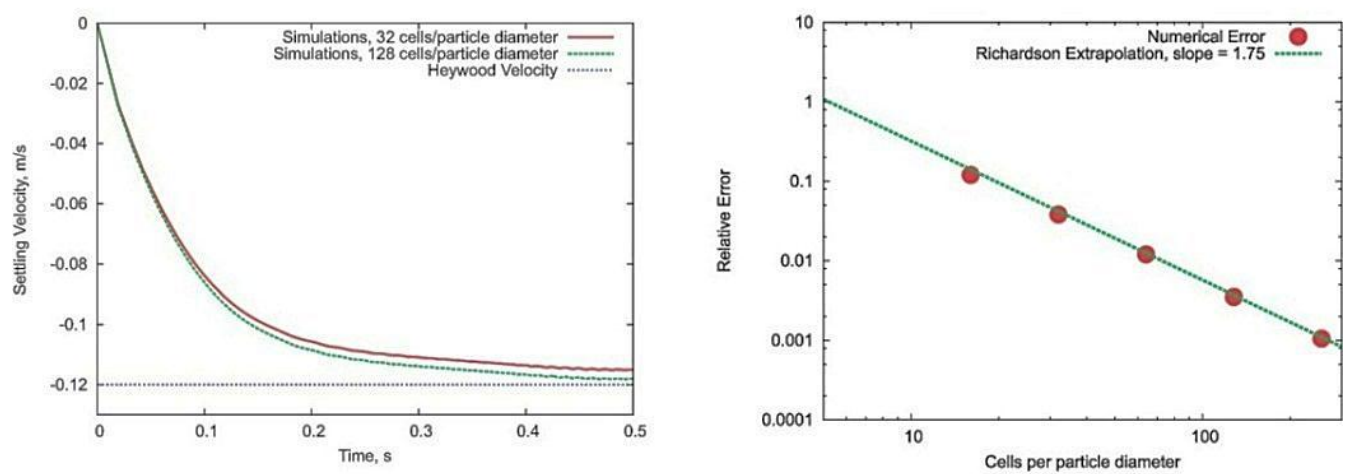

(c) $\operatorname{Re}=500$

Figure 62. Comparison of settling velocity for falling particle example (3D) with analytical Stokes solution Stokes (1966) and Heywood tabulated data Heywood (1948) for different Reynolds numbers (left column). Convergence study for different Reynolds numbers (right column).

Figure 63 shows the patch structure for a numerical solution with adaptive mesh refinement (AMR). The refinement is chosen in zones with maximum vorticity which are shown by color. An adaptive computation employs a total of two levels, with a refinement ratio of two. A reasonable code performance and adaptive speed up with factor of 3.2 is achieved in this example. Our parallel data managing and AMR implementations done similar to algorithm 
described in Griffith et al. (2007, 2010). We do not present detail performance study for AMR technique since it can be found in Griffith et al. (2010).

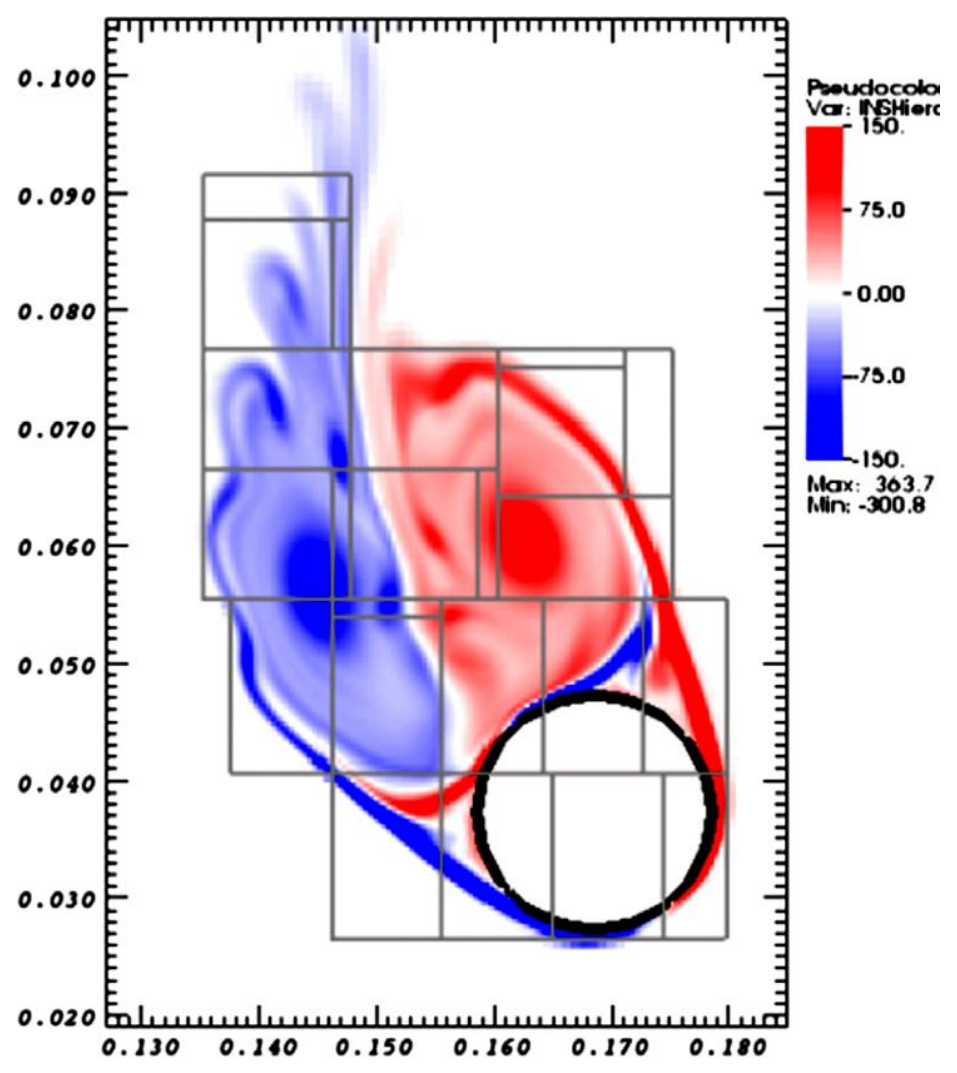

Figure 63. AMR grid in simulations of falling particle in a Newtonian fluid.

Instead, we investigate a code performance as a function of the number of processors, resolution and the number of particles. The summary is presented in Table 6.

Table 6 Time per CPU in seconds to perform a single computed time step as a function of the number of processors and the resolution for the falling particle.

\begin{tabular}{|cccc|}
$\begin{array}{c}\text { Processors/ } \\
\text { Resolution }\end{array}$ & $128 \times 384$ & $\mathbf{2 5 6} \times \mathbf{7 6 8}$ & $512 \times 1536$ \\
\hline $\mathbf{2}$ & 0.578 & 3.76 & 21.8 \\
\hline $\mathbf{4}$ & 0.297 & 1.92 & 11.4 \\
\hline $\mathbf{8}$ & 0.194 & 0.97 & 4.93 \\
\hline $\mathbf{1 6}$ & 0.136 & 0.57 & 2.41 \\
\hline
\end{tabular}

For the falling particle example we use the 2D benchmark configuration described in this section with changing the resolution and the number of processors (2, 4, 8 and 16). For the falling particles example we keep the same parameters (the particle diameter, fluid and particle densities) but increase the computation domain up to $0.2 \times 0.2 \mathrm{~m}$. This time, we investigate the 
code performance with the fixed grid resolution $512 \times 512$ and changing the number of particles $(1,400,1600$ and 6400$)$ and the number of processors $(2,4,8$ and 16). The solid volume fraction is equal to 0.0001 for 1 particle, 0.04 for 400 particles, 0.16 for 1600 particles and 0.64 for 6400 particles. Table 6 demonstrates that the overall code performance and scalability are quite reasonable. It is important that the efficiency of the code does not reduce significantly with increasing the number of particles (less than 10\% difference between simulations with 1 and 6400 particles), so the particle calculations take small fraction of computational cost of underlying fluid solver.

The examples considered in the previous sections focused mainly on the flow through stationary packed beds. When the particles are not so constrained, the motion of the fluid leads to particle motion, and the moving particles interact with each other as well as with the fluid leading to complex flow patterns that depend significantly on the packing density and particle contact physics. For highly viscous fluids, viscous forces could prevent contact between particles, but for most applications, these forces are insufficient to prevent particle contacts. To account for particle-particle interactions, separately applied collision forces similar to those used in the distinct element method (DEM) (Cundall and Strack 1979) are implemented in the code.

To validate the code performance and the particle contact physics algorithm, we performed simulations of a representative experiment conducted by Mike Laufer under the guidance of Professor Per F. Peterson at the U.C. Berkeley Thermal Hydraulics Laboratory. The original experiment was done in PREX-1 facility (Bardet et al. 2007) with some modifications. The modifications included a lowering of the discharge cone and a shortening of the discharge chute in order to accommodate the free release of pebbles. Also, the experiment was done without circulation water through the pebble bed.

The experimental configuration (Figure 64) consists of 2,500 polypropylene pebbles, initially suspended in a vertical water column that was discharged through a narrow opening. The pebble reservoir is $40.6 \mathrm{~cm}$ in diameter, while the narrow opening has a diameter of $10.2 \mathrm{~cm}$. A conical region, with a $45^{\circ}$ cone angle, connects the pebble reservoir to the narrow opening. The surface of the cone has been perforated to allow back flow of water into the reservoir as the pebbles were evacuated. The pebbles were all the same size, with a diameter of $2.5 \mathrm{~cm}$ and a density of $0.843 \mathrm{~g} / \mathrm{cm}^{3}$. The bottom boundary of the container is closed, and a physical barrier is placed in the narrow opening to inhibit pebble motion such that, at the beginning of the experiment, both the pebbles and water are at rest.

The experiment is initiated by removing the physical barrier, thus allowing the pebbles to move upward through the water column. As the pebbles flow out of the lower reservoir, the 
evacuated volume is filled with water flowing into the reservoir through the porous conical section. In the simulations, the holes in the conical section were not modeled explicitly.

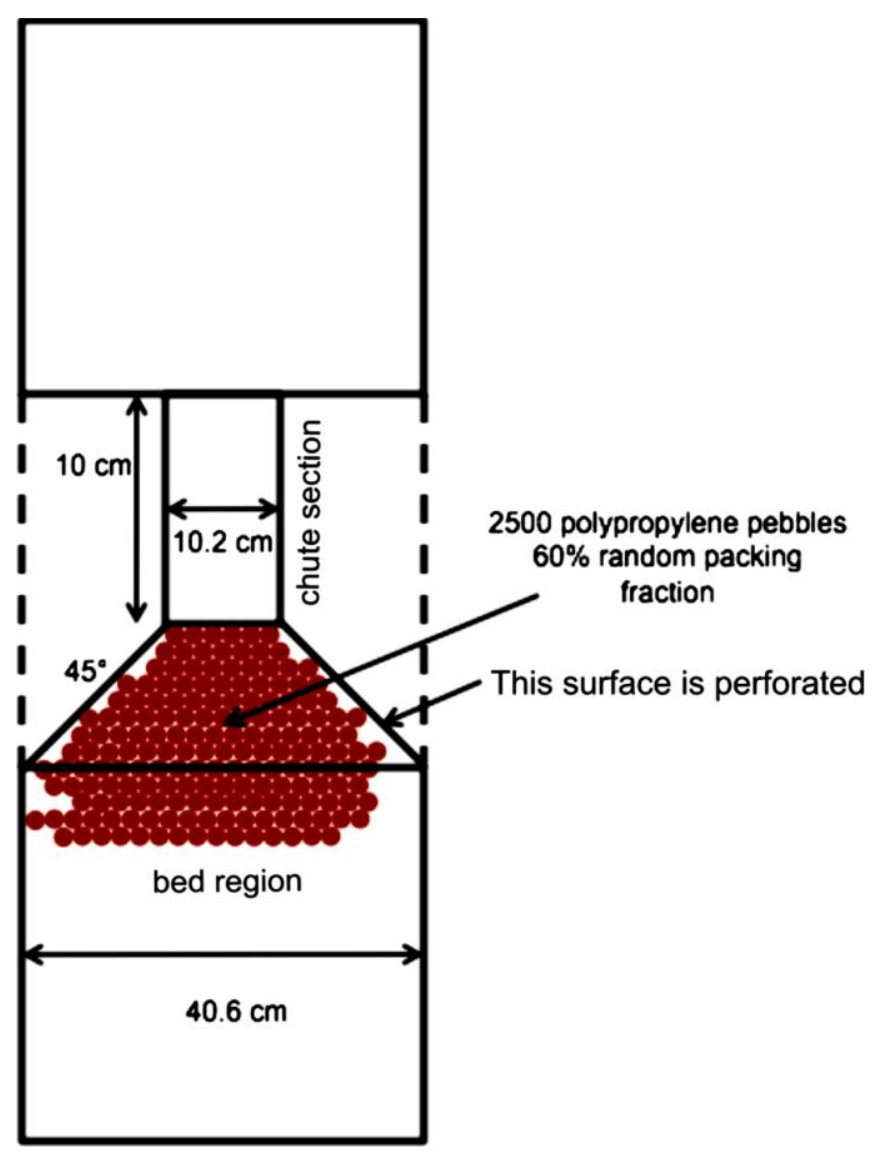

Figure 64. Schematic description of experimental setup performed at the U.C. Berkeley Thermal Hydraulics Lab.

Instead, the effect of the holes was taken into account by allowing the conical surface to physically constrain pebble motion while at the same time be transparent to fluid flow. We employ $768 \times 512 \times 512$ grid resolutions for the finest level, which corresponds to 32 cells per particle diameter. The maximum Reynolds number in the simulations based on the particle diameter was about 5,000. A maximum adaptive time step with Courant number 0.5 was chosen. We also decrease Courant numbers up to 0.05 to study effects of changing the stiffness parameter. It should be noted that we use the same contact parameters or both particleparticle and particle-wall collisions in this example.

The comparisons between simulation results and experimental data are presented in Figure 65 and Figure 66. The overall agreement is very good, though there are some discrepancies. Initially, simulation show that the pebbles move faster in the central region, and slower near the wall with some of the pebbles near the wall moving in a downward direction. This can be 
explained by the fact that the counter fluid flow is not distributed uniformly through the cone section. There could be several reasons for this behavior. One reason may be that the holes in the conical section were not modeled explicitly. Another, more likely reason, is that our initial packing was not ideally conformed to the boundary of the container, thus leading to higher porosity near the skin of the container than in the interior of the pebble bed, thus leading to higher fluid flow rates near the outer boundary of the cylinder. However, it appears that those local fluctuations do not affect the integral characteristics of the flow field. A comparison of the simulated evolution of the bed bottom position with that observed during the experiment is shown in Figure 66.

The bed bottom position was taken as the lowest pebble position in the central cylindrical slice of radius $5.1 \mathrm{~cm}$. We compute the averaged pebble mass flow as

$$
q(z)=\rho_{s} \frac{\sum u(x, y, z) V_{s}(x, y, z) \Delta x \Delta y}{\sum V_{s}(x, y, z) \Delta x \Delta y}
$$

where $\rho_{s}$ is the pebble density, $u(x, y, z)$ is the flow velocity and $V_{s}$ is the volume of fraction of a solid domain in a given point $(x, y, z)$. The pebble mass flux was found to be $4 \mathrm{~kg} /\left(\mathrm{m}^{2} \mathrm{~s}\right)$ in the bed region and about $60 \mathrm{~kg} /\left(\mathrm{m}^{2} \mathrm{~s}\right)$ in the chute section.

(a)

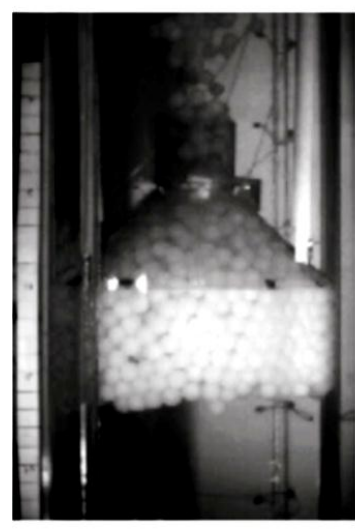

Experiment

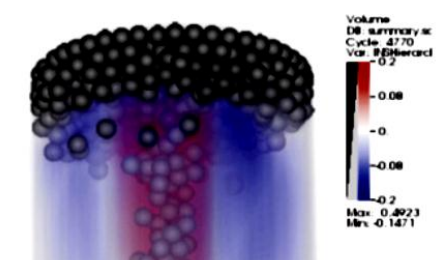

(b)

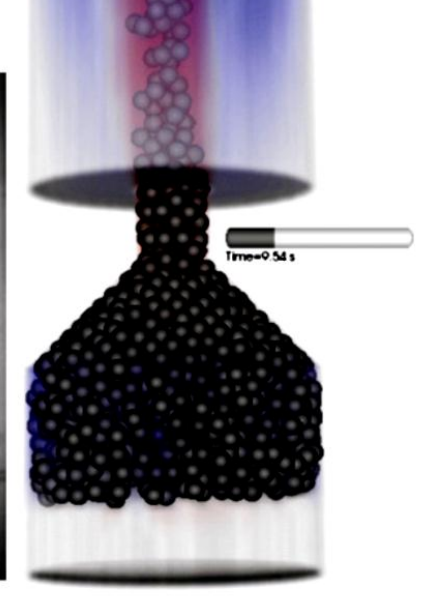

Simulations

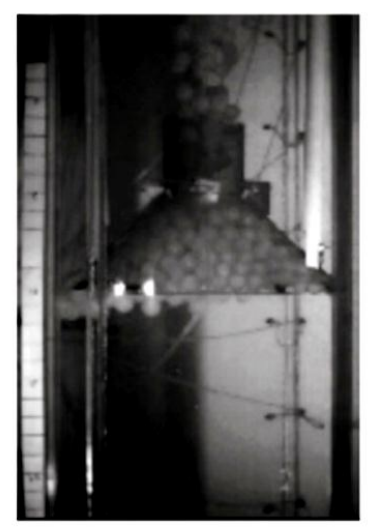

Experiment

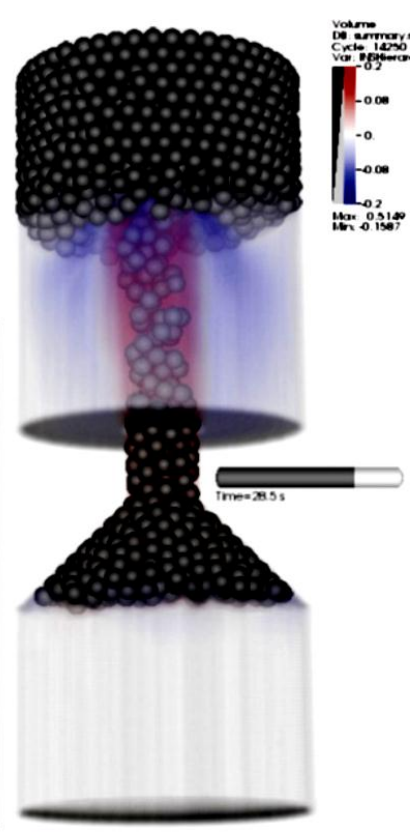

Simulations

Figure 65. Comparison of the pebble flow in experiment and simulations at time $9.5 \mathrm{~s}$ (left panel) and 28.5 s (right panel). 


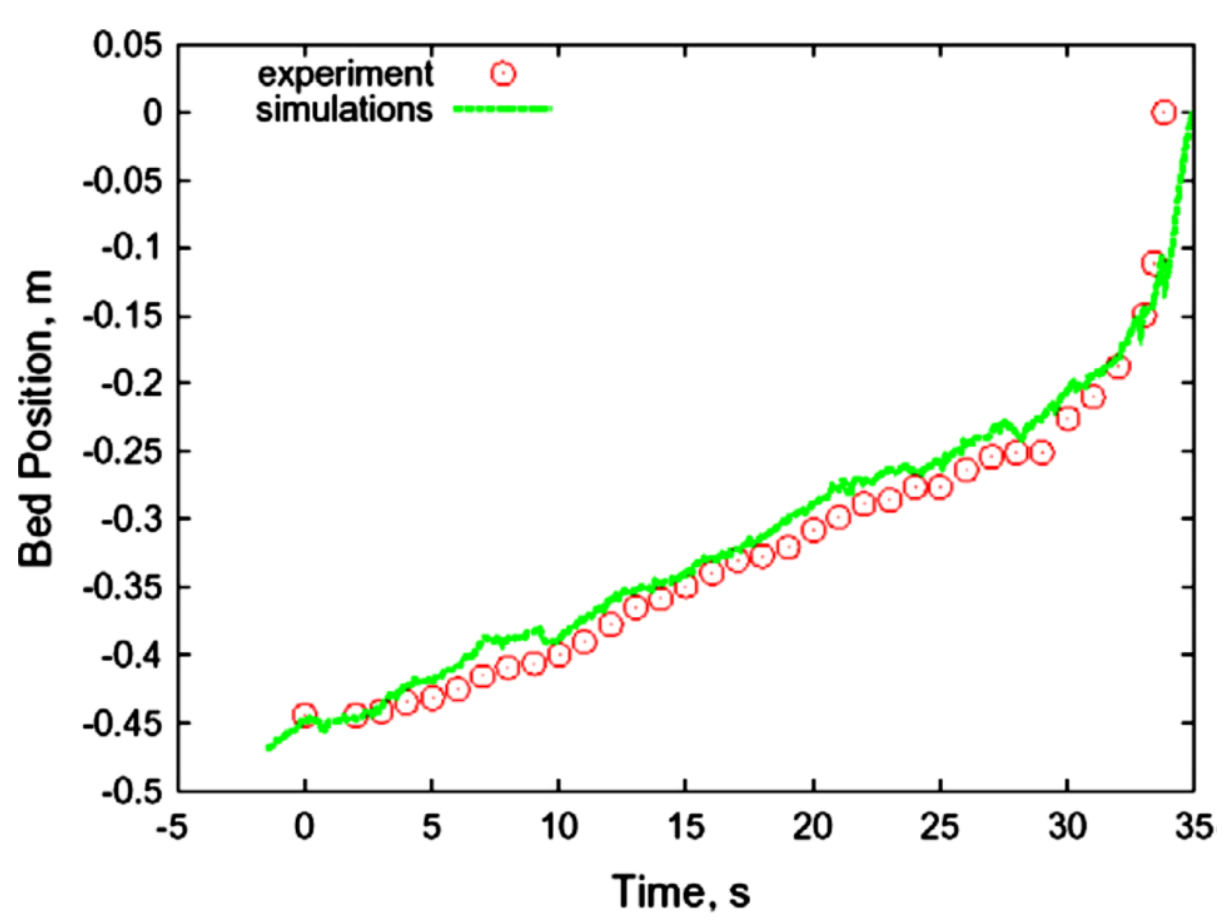

Figure 66. Comparison between experiment and simulations for the bed position below the chute outlet.

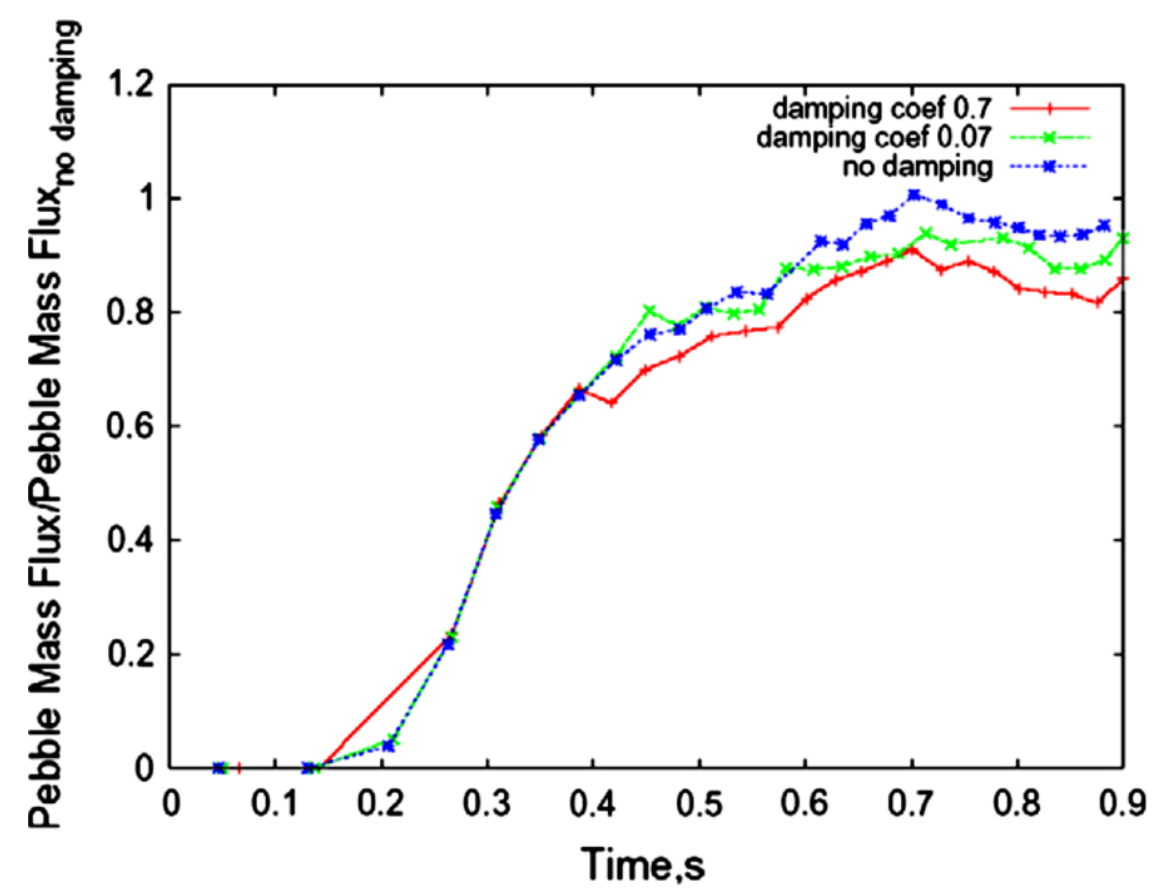

Figure 67. Effect of the damping coefficient on the pebble mass flux in the chute section. The pebble mass flux is shown relative to the pebble mass flux with no damping. Note that the damping coefficient is connected to the restitution coefficient Tsuji et al. (1992). Damping coefficient 0 corresponds to no damping and restitution coefficient 1 , damping coefficient 1 corresponds to fully damped situation with restitution coefficient 0 . 
We performed numerous parametric studies for different boundary conditions and contact model parameters. The most important ones are presented and discussed. The averaged pebble mass flux appears to be not very sensitive to changes in the damping coefficient (Figure 67). According to Tsuji et al. (1992) the damping coefficient is related to the restitution coefficient (the ratio between particle velocities before and after collision). We compare results with no damping (damping parameter 0 ), damping parameters 0.07 and 0.7 and the maximum difference was less than $15 \%$. Our numerical simulations showed that this parameter has a significant effect on the results, and should be chosen very carefully. The characteristic time step for fluid flow could be hundreds of times larger than the time step required to simulate contact interaction between particles based on the stiffness estimated from the physical properties of the particles. If we use real stiffness and explicit integration of contact forces, the computations become prohibitively expensive. However, as demonstrated below, reasonable numerical solutions can be obtained using a lower stiffness than that computed based on physical properties of the pebbles. According to the Hertzian contact theory Hertz (1882), the relation between the normal force $F_{n}$ and displacement $\delta$ is given by:

$$
F_{n}=K_{H} \delta^{3 / 2}
$$

where $K_{H}$ is the Hertzian stiffness. In the case of two spheres of the same size with radius $r$, $K_{H}$ is expressed by:

$$
K_{H}=\frac{\sqrt{2 r} E}{3\left(1-\sigma^{2}\right)}
$$

where $E$ is the Young's modulus and $r$ is the Poisson ratio of the particles. For $E=3 \mathrm{GPa}$ and $r=$ 0.33, the Hertzian stiffness is 1.78 . Since in Eq. (61) the normal contact force is proportional to the volume of the overlapping region, our computational stiffness can be related to the Hertzian stiffness as

$$
K_{H}=k_{n} \pi r \delta^{1 / 2}
$$

The time step required for stable solutions is given as $\Delta t=2 / 5 \pi \sqrt{\frac{m}{K_{H}}}$ (Tsuiji et al. 1992), which for the case considered here is $\sim 10^{5} \mathrm{sec}$. The maximum time step used in our calculations was $10^{-3} \mathrm{sec}$ that corresponds to Courant number 0.5 , thus implying that the minimum 
computational stiffness was in 10000 times smaller than the theoretical value. This agrees well with rough estimation of the stiffness based on the displacement $d$ equal to $1 \%$ of the particle diameter. The simulation results used in the comparisons with experimental data presented here were obtained with a stiffness factor (the ratio of the computational stiffness to the theoretical value) of 0.01 .

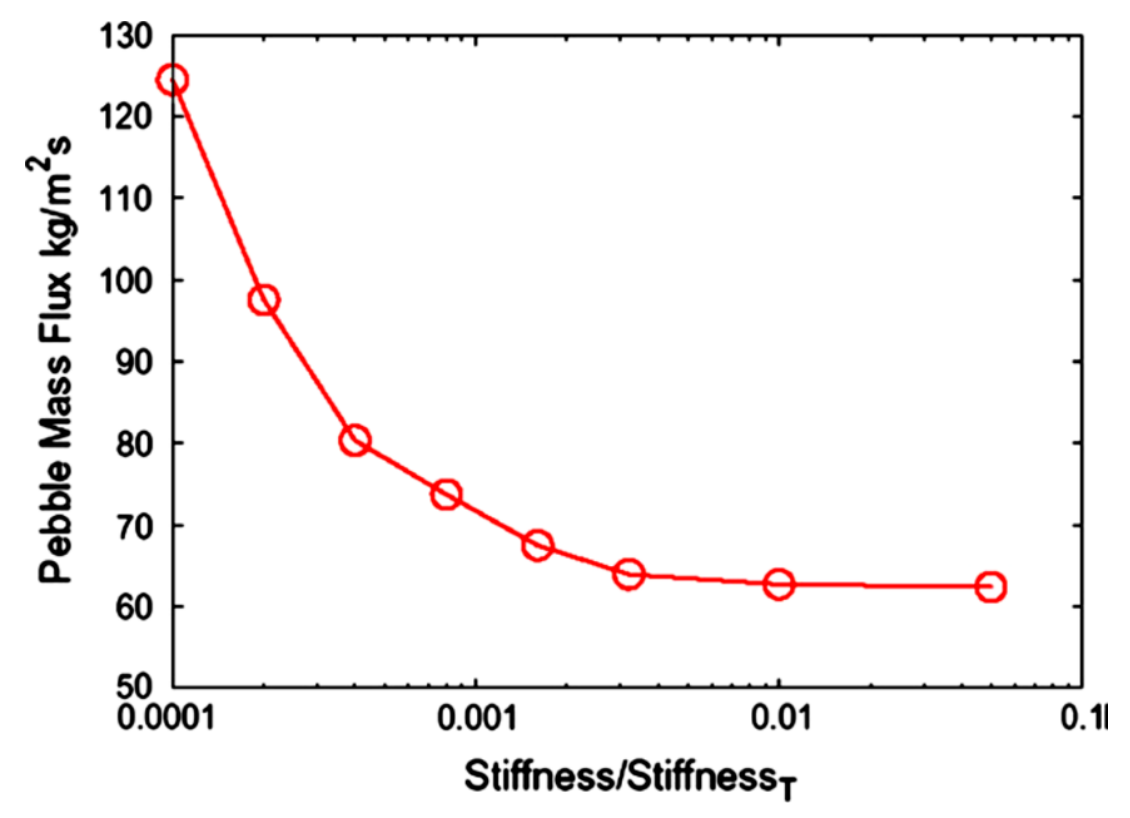

Figure 68. Effect of the stiffness coefficient on the pebble mass flux. The axis shows normalized numerical stiffness using theoretical Hertzian value stiffness $\mathrm{s}_{\mathrm{T}}$.

As indicated in Figure 68 further increase in the computational stiffness does not significantly change the simulated pebble mass flux. Therefore the simulations based on a smaller stiffness (in 100 times smaller) than the actual one can be used to predict pebble flow in a considered regime within a bearable computational time. However using too small stiffness leads to unrealistic flow patterns. This is a quite common problem for DEM models. Although the inter particle forces in reality are intrinsic physical property of the material many researchers have found that using the "softer" particle representation to conserve computational time does not reduce the qualitative nature of the simulated behavior (Li et al. 2003, Asmar et al. 2002, Tuley et al. 2010).

To simulate the fluid particle systems Marshall (2009) proposed to use three-time step procedure consisting of a fluid time step, a particle time step and collision time step. However since our fluid and particle equations are tightly coupled this approach does not seem applicable in our case. Therefore we prefer to use "softer" material and perform parametric studies for different stiffnesses to find an applicable one for considered problem. 


\section{COMPARISON OF SIMULATIONS WITH EXPERIMENTAL DATA}

The ERDC laboratory experiments were designed to produce data for evaluation grain scale simulations of fine sediment transport through filter media. The constant head flow chamber is designed to constrain the filter media and pass flow of a fine-soil suspension through the media. The flow chamber is constructed of acrylic sheeting with inside dimensions of $10 \times 10 \mathrm{x}$ $20 \mathrm{~cm}^{3}$. Flow is delivered to the flow chamber from a constant head tank, which is supplied with flow by a pump in the supply reservoir. Constant head is maintained by means of a vertical riser (2-inch PVC) which permits any excess pump flow to return to the supply reservoir. The experiment also includes a degassing system, implemented to prevent gas precipitation on the filter media. The filter media for the initial experiment consists of 1500 micron soda lime glass spheres. Base particles for this experiment were 300-400 micron soda lime glass beads. The pressure difference during the experiment was measured with a differential pressure sensor connected to pressure ports $10 \mathrm{~cm}$ apart, centered on the $20 \mathrm{~cm}$ length of the flow chamber (Figure 69).

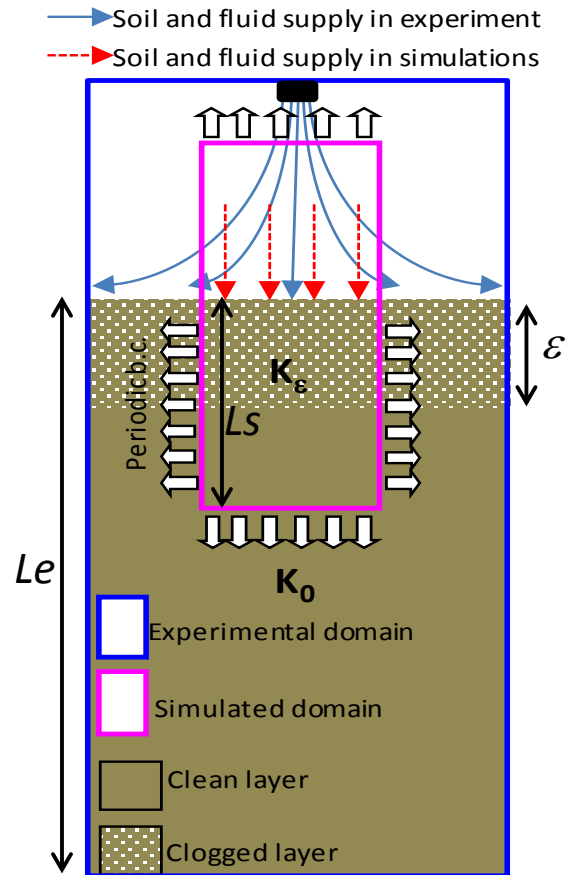

Figure 69. Schematic description of experimental and numerical setups.

To reproduce experimental chamber we use the periodic packing of $1.5 \times 1.5 \times 1.5 \mathrm{~cm}^{3}$ of large filter particles in all simulations. The periodic random distribution of spheres is generated using Donev et al. (2005) algorithm. This packing of porosity $\varphi_{1}=0.62$ is placed in a rectangular box. Then, small particles of porosity of $\varphi_{2}=0.9$ is placed randomly above this layer. The simulations 
are performed with periodic boundary conditions in horizontal and vertical directions. Base soil particles arriving at the bottom of the packing are deleted to avoid multiple particle entrances into domain. Gravity is directed downward. Pressure drop through the water column is simulated by applying the equivalent body force. The physical parameters for fluid and solid are listed in Table 7.

Table 7. Physical parameters for fluid and solid.

\begin{tabular}{|ll|}
\hline Parameter & Value \\
\hline Particle density & $2500 \mathrm{~kg} / \mathrm{m}^{3}$ \\
\hline Fluid density & $1000 \mathrm{~kg} / \mathrm{m}^{3}$ \\
\hline Fluid viscosity & $1 \times 10-3 \mathrm{~N} / \mathrm{m}^{2}$ \\
\hline Coefficient of friction & 0.0 \\
\hline Stiffness & $5000 \mathrm{~N} / \mathrm{m}$ \\
\hline Filter diameter & $1500 \mathrm{microns}$ \\
\hline Base particle diameter & 400 microns \\
\hline Particle shapes & Spherical \\
\hline Porosity of filter layer & 0.38 \\
\hline Porosity of suspended soil particles & 0.9 \\
\hline Pressure gradient (body force) & 0.5 \\
\hline
\end{tabular}

The main difference between numerical and experimental setups is in the particle supply method. In the experiment particles are delivered in the flow chamber by small tubing (Figure 14). Particle concentration is about 0.01. In simulations the base particles are uniformly distributed above the filter layer with particle concentration 0.1 (Figure 70). We used higher concentration of base particles to reduce computational time required to complete one simulation.

Figure 71 shows the results of comparison between experimental data and simulations for fluid mean velocities (derived from the experimental flow rates) and hydraulic gradients. Figure 72 shows the mass fraction of the base material in experiment and simulation. The agreement with experimental data is good. Simulations predict similar flow rate decrease and pressure drop decrease in the clean layer. Since we have different solid mass flow rate in simulation and experiment (due to initial particle concentration and particle supply method) we choose to normalize time using:

$$
T=\frac{d A}{M_{s}}
$$


where $\mathrm{T}$ is time required to fill the volume $d A$, where $\mathrm{A}$ is the area of the flow chamber and $\mathrm{d}$ is the particle diameter, $M_{s}$ is the solid flow rate. We used $T_{1}=8.5 \mathrm{sec}, T_{2}=0.07 \mathrm{sec}$ to normalize plots in Figure 71.
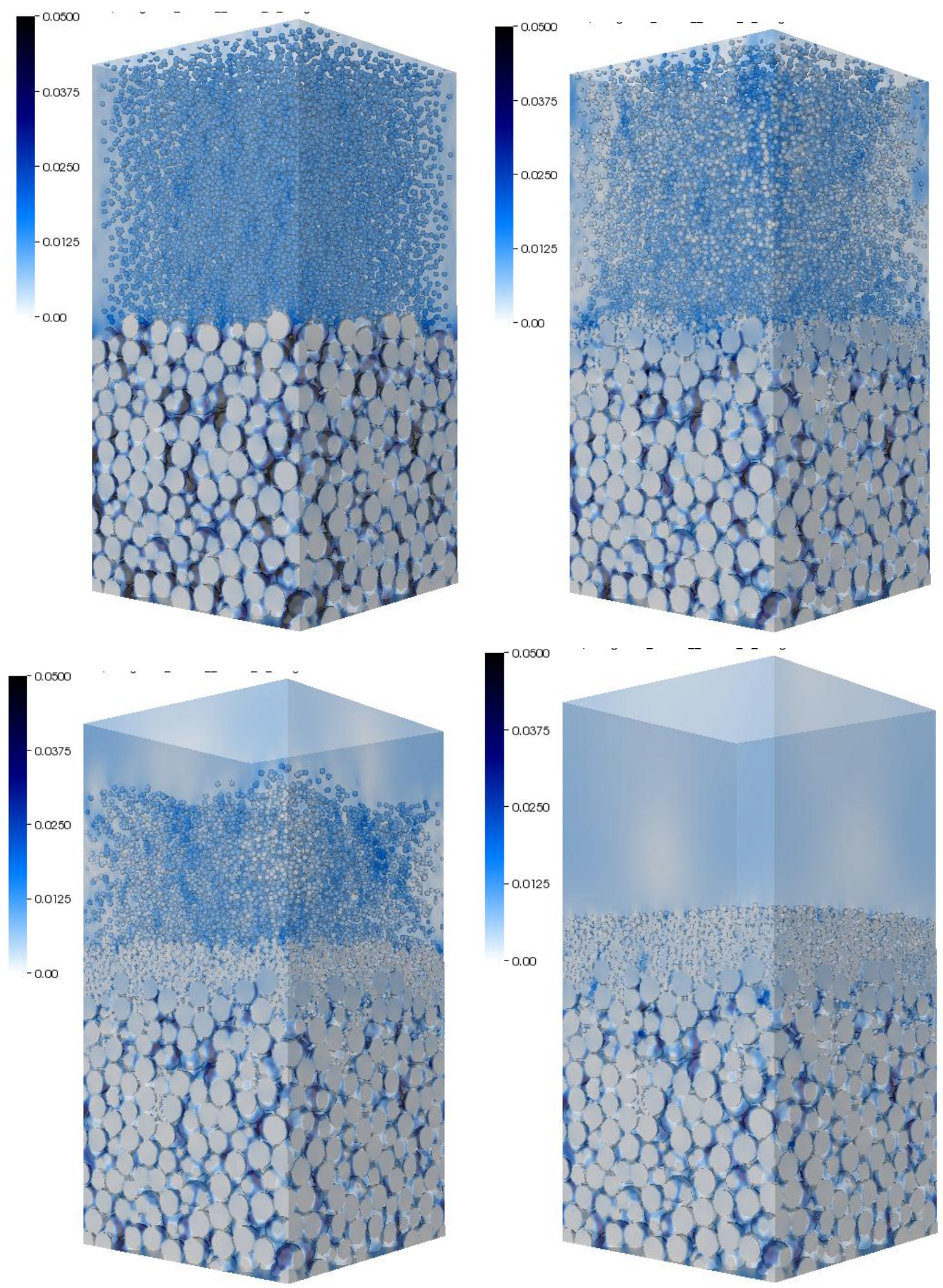

Figure 70. Simulations of base particle clogging in the filter layer. 

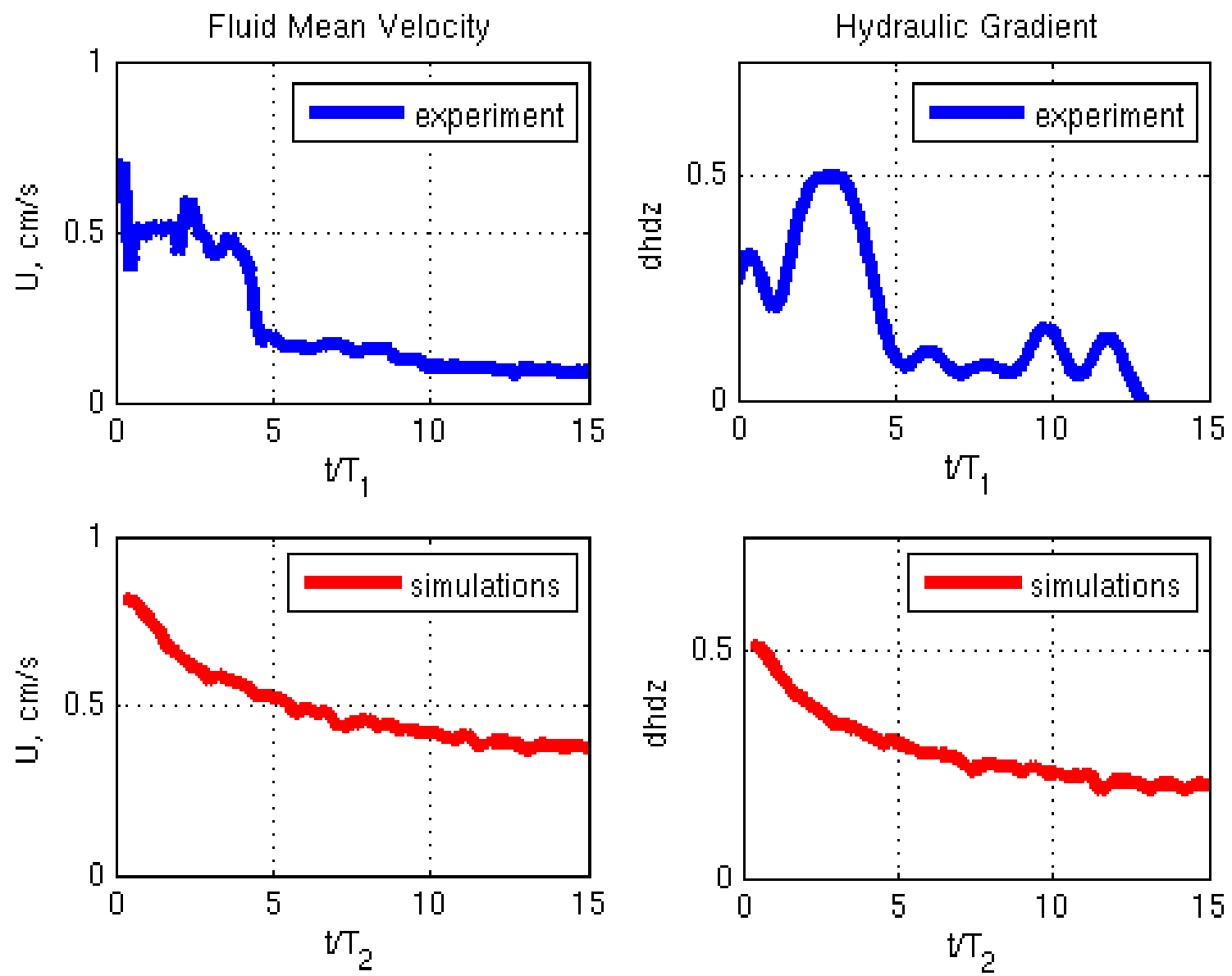

Figure 71. Comparison between simulations and experiment for fluid mean velocity (derived from provided flow rates) and hydraulic gradients in the clean layer.

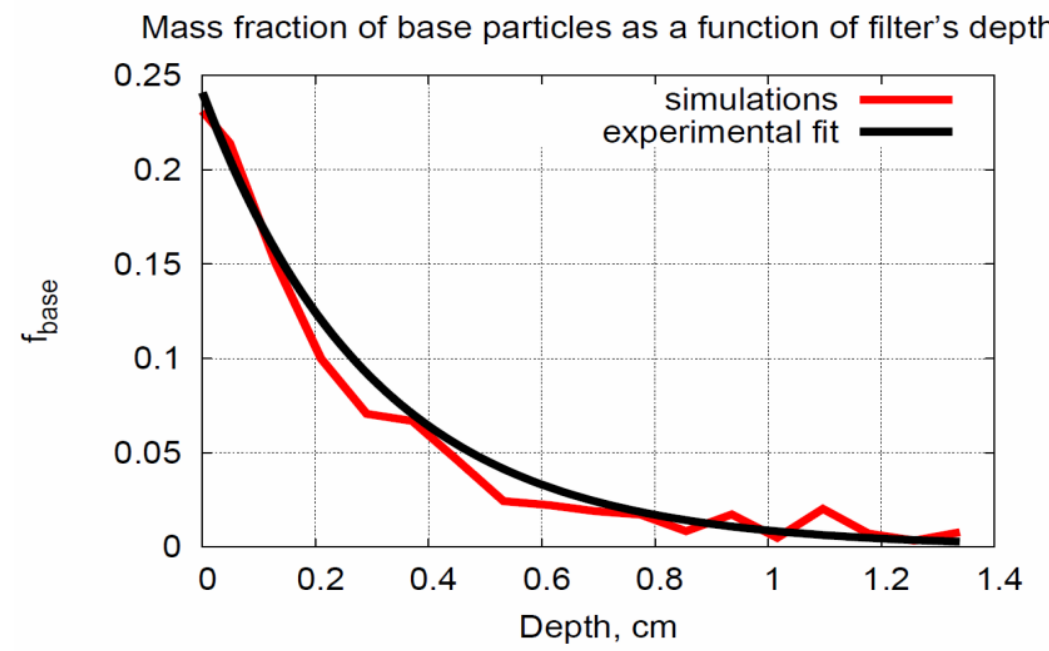

Figure 72. Comparison between simulated and experimental mass fraction of the base particles in the filter layer. 
However there are still some discrepancies caused by differences between initial setups in the experiment and simulations. One can see that the fluid flow rate decreases gradually in the simulation and it has step-like behavior in the experiment. The sharp decrease in the flow rate in the experiment may correspond to the moment when the chamber surface becomes completely covered by the base particles. Since the base particles' source is uniform in the simulation, the chamber surface is uniformly covering by particles until they clog the filter. As a result the flow rate decay is gradual.

To confirm this explanation we perform the additional set of simulations with the partial distribution of the base particles in such a way, that the base particles cover only $1 / 4$ of the space above the filter layer (Figure 73). Figure 74 shows that the flow rate remains nearly uniform until the base particles cover the filter surface and then gradually reduces with time.

It is worth noting that Figure 71 shows good agreement between simulated and experimental base particles distribution in the filter. Therefore we may conclude that the particles' delivering method into the filter is not so important for the final base particle distribution in the filter. For more consistent comparison we estimate the permeability of the clogged layer $\left(k_{\varepsilon}\right)$ and the clean layer $\left(k_{0}\right)$ in both simulations and experiment. The pressure drop was measured only in the lower layer of the filter located above the clogged layer. Therefore the pressure drop in the upper clogged layer is unknown in the experiment. However these quantities can be estimated based on the scaling analysis shown in Figure 75 and the fact that the clogged layer depth $\varepsilon$ is known. According to Darcy's law:

$$
\begin{aligned}
& \Delta p_{1}=f_{1 s} \varepsilon \mu / k_{\varepsilon} \\
& \Delta p_{2}=f_{1 s} \mu\left(\varepsilon / k_{\varepsilon}+(L s-\varepsilon) / k_{0}\right) \\
& \Delta p_{3}=f_{1 e} \varepsilon \mu / k_{\varepsilon} \\
& \Delta p_{4}=f_{1 e} \mu\left(\varepsilon / k_{\varepsilon}+(L e-\varepsilon) / k_{0}\right)
\end{aligned}
$$

where $f_{1 e}$ and $f_{1 s}$ are the final flow rates in experiment and simulation correspondingly.

The permeability of the clean layer can be found directly from the measured hydraulic gradients and the initial flow rates $f_{0 e}$ and $f_{0 s}$ as: 


$$
\begin{aligned}
& k_{o}=f_{o e} L e \mu / \Delta p_{4} \text { (in experiment), } \\
& k_{o}=f_{o s} L s \mu / \Delta p_{2} \text { (in simulations), }
\end{aligned}
$$

From the experimental data we know:

$$
A_{1}=\left(\Delta p_{4}-\Delta p_{3}\right) /(L e-\varepsilon)
$$

Therefore the permeability of clogged layer can be estimated as

$$
\begin{aligned}
& k_{\varepsilon}=f_{1 e} \varepsilon \mu /\left(A_{0} L e-A 1(L e-\varepsilon)\right) \text { (in experiment), } \\
& k_{\varepsilon}=f_{1 s} \varepsilon \mu / \Delta p_{1} \text { (in simulations). }
\end{aligned}
$$

Table 8 summarizes the results of comparison and we see that both simulations and experiment predict the same final permeability of the clogged layer. This fact confirms the consistency of numerical simulations.

Table 8. Comparisons between experiment and simulations.

\begin{tabular}{|lcc|}
\hline Initial hydraulic gradient & Experiment & Simulations \\
\hline Final hydraulic gradient & 0.5 & 0.5 \\
\hline Initial flow rate $f_{0}, \mathbf{c m} / \mathbf{s}$ & 0.1 & 0.2 \\
\hline Final flow rate $f_{1}, \mathbf{c m} / \mathbf{s}$ & 0.5 & 0.8 \\
\hline Clogged layer depth $\mathbf{\varepsilon}, \mathbf{c m}$ & 0.1 & 0.4 \\
\hline Filter depth $\mathbf{L}, \mathbf{c m}$ & 1.2 & 1.2 \\
\hline Permeability of clogged layer $k_{\varepsilon}, \mathbf{c m}^{\mathbf{2}}$ & 20 & 1.5 \\
\hline Permeability of clean layer $k_{0}, \mathbf{c m}^{\mathbf{2}}$ & $8.07 \mathrm{E}-6$ & $8.01 \mathrm{E}-6$ \\
\hline
\end{tabular}



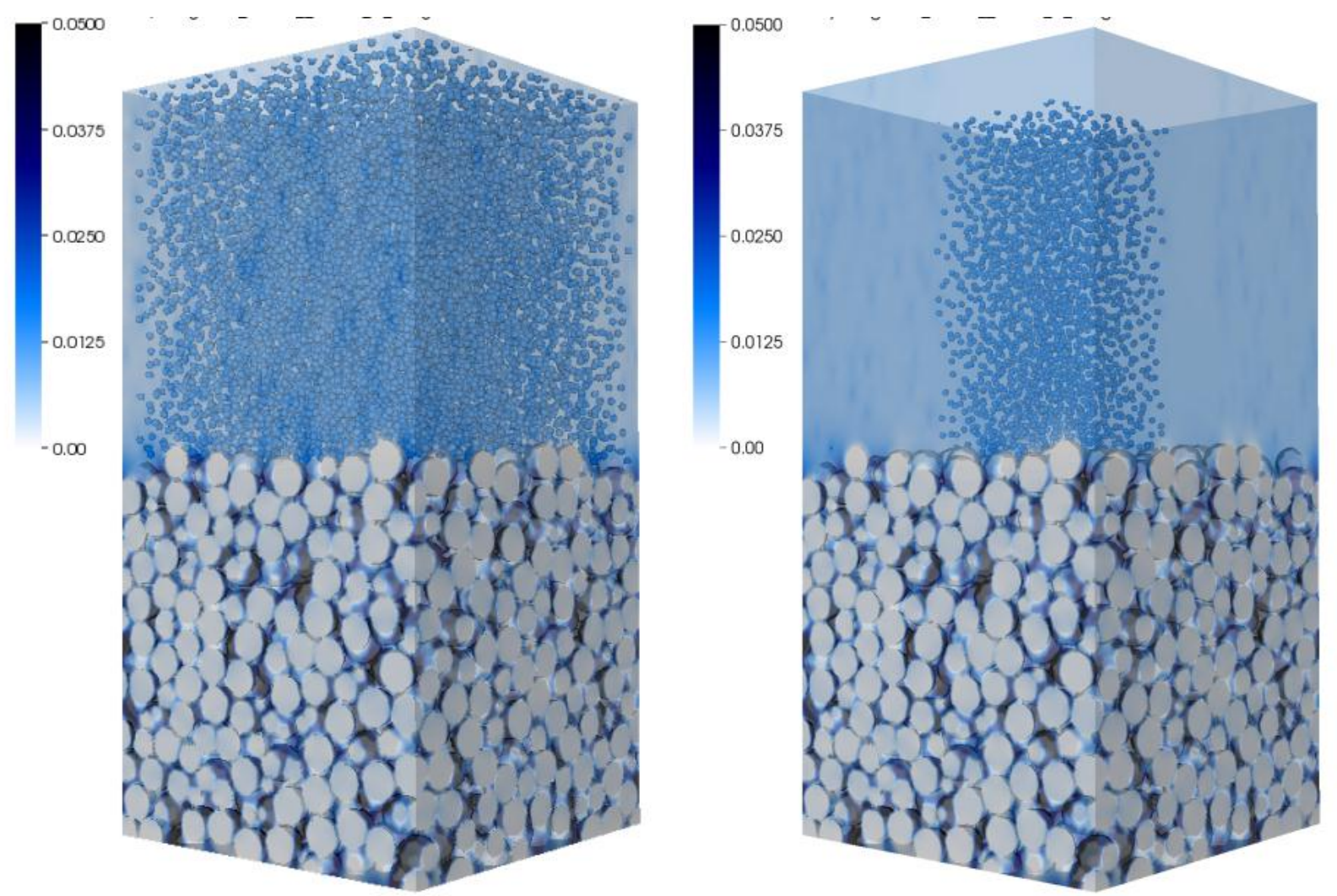

Figure 73. Uniform distribution of the base particles (left) and partial distribution of the base particles (right).
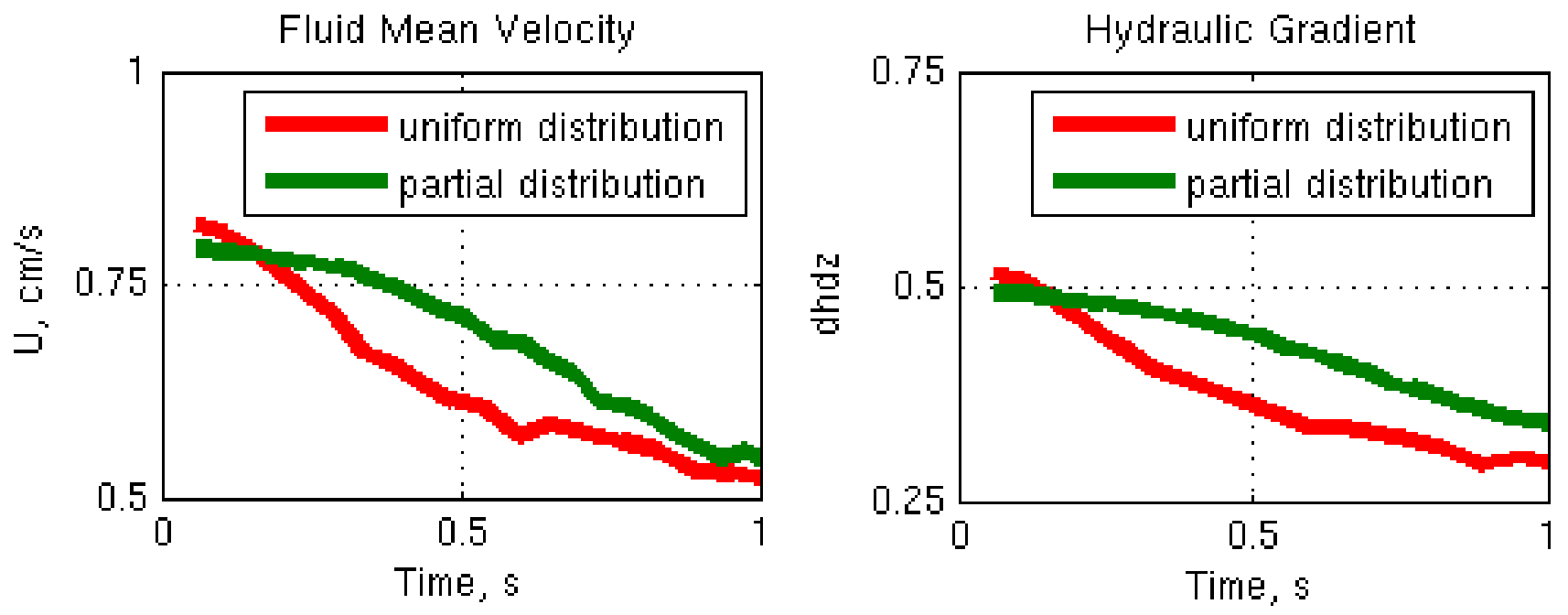

Figure 74. Comparison of the fluid mean velocity evolution (left) and hydraulic gradient in clean layer (right) for uniform and partial base particle sources. 


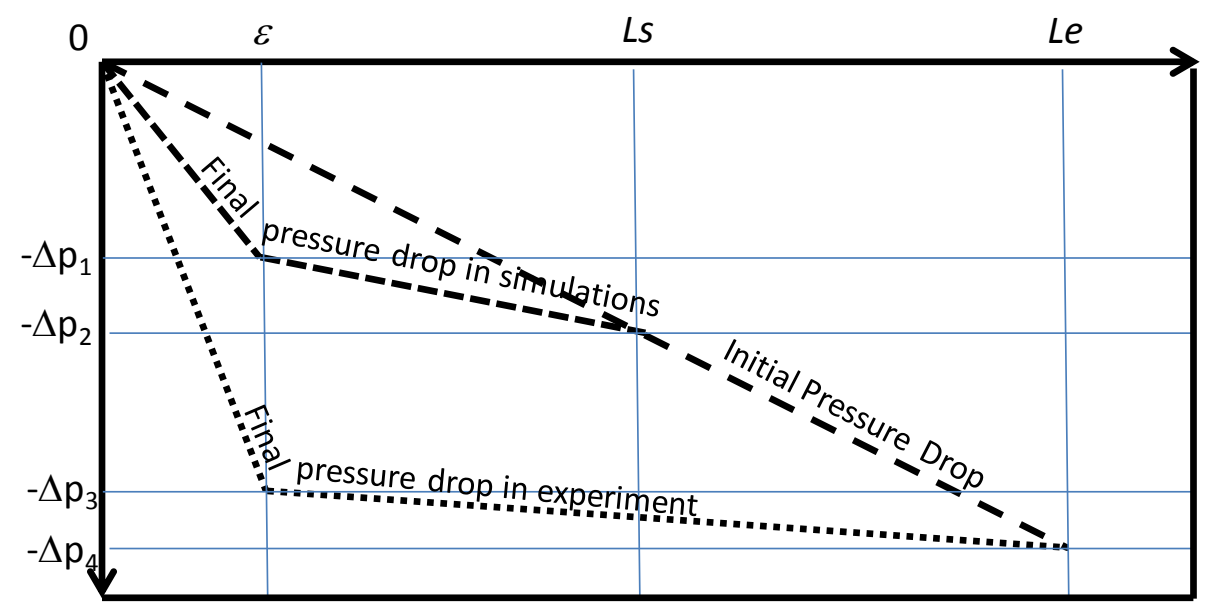

Figure 75. Schematic description of the pressure drops in simulations and experiment.

\section{CONCLUSIONS}

We have outlined a preliminary computational methodology to address a difficult multidisciplinary problem covering a wide range of spatial and time scales. The approach focuses on evaluating the consequences of dynamic loading of an interface between the end monoliths of a concrete gravity dam and where they meet up with and are supported by wraparound sections embankment dams. We consider aspects of the problem which involve significant, possibly non-laminar, fluid flow in opened cracks. The initial approach to the problem involves Eulerian-Eulerian multiphase methods, a phenomenological approach that requires multiple closure models. We used functional parameterizations of these models available in the literature, while further constraining material parameters with HET and JET experiments for particular soil types.

Dam embankments have complicated zoning, with an impermeable core, sand and gravel filters. Different particle sizes are necessary for effective design, but create additional computational challenges. Interphase momentum exchange terms are not well characterized for flows with wide particle size distributions. We overcame these difficulties with high fidelity simulations resolving flow around the individual particles. To evaluate internal erosion processes, both approaches can be combined in a single simulation when bigger particles are directly resolved and smaller particles are represented by an Eulerian phase. Validation of simulations with scaled experiments of realistically zoned dams helped building confidence in our computational tools, so they can be used to better assess the performance of soil/concrete interfaces when subjected to deformations caused by dynamic loads.

We have presented a distributed Lagrange multiplier algorithm for particulate flows. The idea of the method is to use operator-splitting technique to solve the fluid equations in the entire 
domain first, and then correct the flow inside the rigid domain using Lagrange multipliers. The parallel implementation of the algorithm is done using SAMRAI library. Previous works include collisions based on elastic forces that prevent particles from overlap. We extend this approach by incorporating DEM methods for inelastic, frictional contact forces. Code validation was done by comparing numerical results with known experimental and empirical data for a falling sphere in a Newtonian fluid, flow through the stationary packed beds, and pebble release through a narrow opening. We performed numerous convergence tests for different applications and found that for the low and moderate Reynolds numbers, the convergence of the method is close to the second-order. For the high Reynolds numbers, the convergence becomes slightly slower and could be explained by deficiencies in the flow representation near the rigid walls, in particular, the velocity interpolation in the mixed cells where both fluid and solid coexist and necessity to resolve turbulent structures in the fluid domain. The overall performance and accuracy of the code is very good and promises to be a valuable tool both for simulations of flow involving up to thousands of particles as well as for calibrating the phase-coupling relationships in two-fluid continuum simulation models.

We have also implemented a linear and non-linear filtration using single (Type I) and dual (Type II) continuum fine population models to simulate the impact of mobilized fines on the fate of flow and stability of embankments. The model solves simultaneously, the flow, attachment and washout of fine particles within a heterogeneous porous media. The model uses state of the art finite element method with adaptive mesh refinement that not only captures the interface between water dense with fines/clear water but also the non-linearity of the free surface itself. We have conducted several dislocations scenarios and their subsequent fines releases. Through these simulations, one concludes -- regardless the type of the adopted transport of fines model -- the attachment kinetic isotherm is the key function of the model. Initial results show that the retention of fines can be as low as $30 \%$ and as high as $70 \%$. More meso-scale experiments are needed for typical embankment soils to further reduce the uncertainty within the model. Furthermore, more parametric studies need to be conducted to assess the sensitivity of the kinetic isotherm parameters on the stability of the embankment. It is worth mentioning that the model, for the more realistic, non-linear kinetic isotherms has predicted a self-rehabilitation of the breached core with retention of $50 \%$ of the mobilized fines using a very conservative filtration length of $10(1 / \mathrm{s})$. A more realistic value should exceed the assumed one, thus a retention exceeding 50\% for Type I and 70\% for Type II retention models, respectively.

We have also investigated soil erosion processes for different particle sizes and shapes (spherical particles and irregular particles) at the grain scale. We compared threshold values for the critical shear stresses; shear stress velocities and mean flow velocities against experimental data and found favorable agreement in the particle size range 50-800 microns. For smaller 
particle sizes cohesion forces become important and need to be included in our future simulations.

Free parameters of a general constitutive erosion law have been investigated as a function of particle and fluid properties. It was found that irregular particles are eroded significantly faster than spherical particles. Both changes in particle compaction and in the lift and drag forces due to the particle shape contribute to this, but an impact of particle compaction is more significant. It was found that erosion rates for smaller particles are higher than erosion rates for larger particles in considered range of the particle sizes 50-800 microns. We confirm that linear relationship between particle mass fluxes and shear stresses describes well soil behavior. An appropriate range of parameters for erosion law is established and can be used in large-scale continuum models.

Preliminary simulations of soil trapping in filter layers demonstrate code applicability to the problem of interest. In future, we are planning to validate existing large scale parameterizations for soil transport in filter layers and derive new ones if appropriate. We are planning a more detail comparison study between meso-scale and large-scale simulations for the equivalent soil conditions and problem setups to understand connections between meso and large scales. Future work will include the impact of lateral dispersion, particle properties, and amplitude of the applied hydraulic gradient as well as local heterogeneities in packing on clogging criteria and changes in hydraulic conductivity of the medium.

A set of laboratory experiments have been conducted by ERDC to obtain qualitative and quantitative data for fine particulates passing through granular filter media. The results will be validated against numerical model data.

We have also investigated soil erosion processes for different particle sizes and shapes (spherical particles and irregular particles) at the grain scale. We compared threshold values for critical shear stresses and shear velocities and mean flow velocities against experimental data, and found favorable agreement in the particle size range 50-800 microns. For smaller particle sizes cohesion forces become important and need to be included in our future simulations.

Free parameters of a general constitutive erosion law were investigated as a function of particle properties. It was found that irregular particles are eroded significantly faster than spherical particles. Both changes in particle compaction and in lift and drag forces due to particle shape contribute to this, but an impact of particle compaction is more significant. It was found that erosion rates for smaller particles are higher than erosion rates for larger particles in the considered range for the particle sizes of 50-800 microns. We confirm that a linear relationship between particle mass fluxes and shear stresses well describes soil behavior. An appropriate 
range of parameters for erosion law is established and can be used in large-scale continuum models.

The failure modes of this embankment/concrete gravity dam model at $1 \mathrm{~g}$ and $30 \mathrm{~g}$ 's were almost identical. Both models had a dominate crack 45 degrees from the plane surface of the concrete monolith forming a wedge of material that moved down stream in the $1 \mathrm{~g}$ experiment. The $30 \mathrm{~g}$ experiment had less rotation and corresponding less crest displacement (maximum crest displacements $1 \mathrm{~g}-4.124$ inches, $30 \mathrm{~g}$ 's -2.1 inches) resulting in the wedge of downstream embankment material not moving and forming the horizontal crack. Both models had similar significant cracking with no seepage occurring through the damaged embankment.

During this study we have established the key tools at the grain, meso- and macro- scales and investigated their performances and responses to different functional or scalar key parameters. However, due to time and funding limitations, the upscaling of the key parameters from the grain scale to the macro scale need to be further investigated and implemented for more consistent predictions at the dam scale. Moreover, in order to apply the current tools to real infrastructures (dams and embankment), we need to catalogue and map the initial state (current condition, hydraulic parameters etc...) of the infrastructures themselves. This could be done via non-intrusive geophysical (magnetic, electric, etc...) sensing to map for any preexisting cracks or imperfections that will exacerbate and further compromises the instability of the infrastructures. Uncertainty within the mapped hydraulic and geological parameters cannot be eliminated but only reduced. We would like to conduct uncertainty quantification on the key parameters and perform a probabilistic assessment of the failure of the infrastructures. Through the current study, we believe we have the necessary tools to establish a stochastic framework for dams and embankments failure analysis that could also be used for other DHS structure of interest.

\section{IDENTIFIED GAPS AND PATH FORWARD}

During the course of the current study we have identified several future steps tones for strengthening the already presented tools and prepare them for real case studies.

- Further development of grain scale numerical tools with additional validation experiments. For example all simulations at the grain scale do not take into account the cohesive interactions (forces) between the grains. This effort has been initiated in 2013 .

- Upscaling from grain scale numerical results to dam scale numerical tools. The correlations for filter performance will be upscaled and integrated into the dam/embankment for filter efficiency study at the operational scales. This effort has also been initiated in 2013. 
- Quantitative validation of dam scale numerical tools with appropriately scaled experiments. This step has been already initiated in 2013 and its completion will further strengthen our numerical code reliability at the dam/embankment scale.

- Development of complete soil constitutive model that properly accounts for water without effective stress assumptions. LLNL has invested substantial resource in studying the validity of Terzaghi's postulate of effective stresses under extreme dynamic loads. Assessment of dam resilience under explosive loading will greatly benefit of the currentstate-of-the-art understanding of the explicit physics of fluid/solid interactions rather than effective stress assumptions. This task will rely on several experiments that will be conducted at ERDC.

- Account for probabilistic variation of soil and hydraulic properties, crack initiation, and the propagation of uncertainty on dam failure. LLNL has leading edge uncertainty quantification and propagation tools that can be directly applied to assess the resilience of dams and embankments under conditions of uncertainties. Resilience will be measured in term of probability rather than in a deterministic space.

- Development of dam scale numerical tools that include formation of cracks and slope failures along crack opening. LLNL has already several tools that can be customized for the problem at hand. The step will enable developing contingency plans and design short and long remedies.

We believe that these efforts will bring the project to completion with reliable tools that can be used to assess several real structures around the nation and will set standards for assessing dam/embankment resilience for US Army Corps of Engineers. We project that the suggested efforts will require a combined LLNL-ERDC effort of two years of funding at the rate of 3FTEs.

\section{REFERENCES}

Asmar B.N., Langston P.A., Matchett A.J., Walters J.K. Validation tests on a distinct element model of vibrating cohesive particle systems. Comput. Chem. Eng., 26, 785-802, 2002

Bardet P., An J.Y., Franklin J. T., Huang D., Lee K., Mai A., The pebble recirculation experiment (PREX) for the AHTR. Global, 845-851, 2007

Blasco J., Calzada M.C., Martin M. A fictitious domain, parallel numerical method for rigid particulate flows. J. Comp. Phys., 228, 7596-613, 2009. 
Balay, S., Buschelman, K., Eijkhout, V., Gropp, W., Kaushik, D., Knepley, M., McInnes, L. C., Smith, B., and Zhang, H. PETSc users manual. Tech. Rep. ANL 95/11 - Revision 2.2.1, Argonne National Laboratory, 2004.

Berger, M.J. \& Oliger, J. Adaptive mesh refinement for hyperbolic partial differential equations, J.Comp.Phys., 53, 484-512, 1984

Bohling B. Measurements of threshold values for incipient motion of sediment particles with two different erosion devices. Journal of Marine Systems, 75, 330-335, 2009.

Bonelli S., Marot D. (2008) On the modelling of internal soil erosion. Int. Association for Computer Methods and Advances in Geomechanics, 2008.

Bonelli S. and O. Brivois. The scaling law in the hole erosion test with a constant pressure drop. Int. J. Numer. Anal. Meth. Geomech, 32, 1573-1595, 2008

Bonelli S, Benahmed N., Brivois O. (2006) On modelling of the hole erosion test. ICSE, 3rd International conference on scour and erosion, Amsterdam, 2006.

Bonelli S., and Brivois O. (2007) The scaling law in the hole erosion test with a constant pressure drop. Int. J. for Num. and Anal. Methods. in Geomech., 32(13), 1573-1595.

Brownlie W. Compilation of alluvial channel data: laboratory and field. Tech. Rep. KH-R-43B, California Institute of Technology, Pasadena, California, USA. 1981

Buffington, J. M. and D. R. Montgomery. A systematic analysis of eight decades of incipient motion studies, with special reference to gravel-bedded rivers. Water. Resours. Res., 33(8), 1993-2029, 1997

Brethour, J.M., (2001) Transient 3D Model for Lifting, Transporting, and Depositing Solid material, Proc. 3rd International Environmental Hydraulics, Dec. 5-8, 2001, Tempe, AZ

Bureau G. J. (2003) Dams and Appurtenant Facilities. Earthquake Engineering Handbook edited by Chenh, W.F. and Scawthorn, C. CRS press, $26.1-26.47$.

Chanson, H., Jarny S., Coussot P. (2006) Dam break wave of thixotropic fluid. J Hydr Eng., ACSE, 2006, 132 (3), 280-293.

Cundall P.A. and Strack O.D.L. (1979) A discrete numerical model for granular assemblies. Geotechnique, 29, 47-65. 
Dasani D., C. Cyrus, K. Scanlon, R. Du, K. Rupp, K. Henthorn. "Effect of particle and fluid properties on the pickup velocity of fine particles." Powder Technology, 196, 237-240, 2009.

Donev, A., F. H. Stillinger and S. Torquato, "Neighbor list collision-driven molecular dynamics simulation for nonspherical particles. I. Algorithmic details". J Comp Phys, 202 (2), 734764,2005

Ergun S. "Fluid flow through packed columns."Chemical Engineering Progress, 48, 89-94, 1952

Fell R., MacGregor P., Stapledon D., Bell G. (2005) Geotechnical engineering of dams. Balkema, Leiden, ISBN 041536440.

Fell R., Wan C.F., Cyganiewicz J., Foster M. (2001) The time for development and detectability of internal erosion and piping in embankment dams and their foundations. UNICIV report R-399.

Foster M., Fell R., Spannagle M. (2000) The statistics of embankment dam failures and accidents. Can. Geotech. J., 37(5): 1000-1024.

Glowinski, R., Pan T.W., Hesla T.I., Joseph D.D. (1999). A distributed Lagrange multiplier/fictitiouss domain method for particulate flow. Int J Multiphase Flow, 25, 755794.

Marsily de G. Quantitative hydrogeology. Groundwater hydrology for engineers. Academic Press, 1986

Engineer manual No. 1110-2-1901. Department of the Army, U.S. Army Corps of Engineers, Washington, DC, April, 1993

FLUENT, FLUENT 6.0 User's Guide Book, 2001.

Franzen P. Zum Einflu der Porengeometrie auf den Druckverlust bei der Durchstromung von Porensystemen, I. Versuche an Modellkanalen mir variableem Querschnitt, Rheol Acta, $18,392-423,1979$

Gidaspow, D., 1994: Multiphase flow and fluidization. Continuum and Kinetic theory descriptions. Academic Press, NY.

Glowinski, R., T.W. Pan, T.I. Hesla, D.D. Joseph, Periaux J., 2001: A fictitious domain approach to the direct numerical simulation of incompressible viscous flow past moving rigid bodies: application to particulate flow. Journal of computational physics, 197, p2305-2316. 
Griffith B.E., R.D. Hornung, D.M. McQueen, and C.S. Peskin, 2007: An adaptive, formally second order accurate version of the immersed boundary method. J. Comp. Phys., 223, 10-49.

Griffith B.E., R.D. Hornung, D.M. McQueen, and C.S. Peskin., 2010: Parallel and Adaptive Simulation of Cardiac Fluid Dynamics. In: Advanced Computational Infrastructures for Parallel and Distributed Adaptive Applications. M. Parashar, S. Chandra, and X. Li, eds. John Wiley and Sons.

Hanson G. J., K. R. Cook. (2004) Apparatus, test procedures, and analytical methods to measure soil erodibility in situ. Applied Engineering in Agriculture, 20 (4), 455-462.

Hertz H., 1882: Über die Berührung fester elastische Körper, Journal of Reine und Angewandte Mathematik 92, 156-171.

Herzig, J. P., D. M. Leclerc, and P. LeGoff. 1970. Flow of suspensions through porous media, p. 129-157, Flow through porous media. American Chemical Society, Washington, DC.

Heywood H., 1948: Calculation of particle terminal velocities. J. Imp. Coll. Chem. Eng. Soc.,4, 140-257.

Hjulstrom F. Studies of the morphological activity of rivers as illustrated by the river Fyris, Bull. Geol. Inst., Uppsala 25, 221-527, (1935).

Hornung R. D. and S. R. Kohn “Managing Application Complexity in the SAMRAI Object-oriented Framework", Concurrency Computat. Pract. Exper. 14, 347-368, 2002.

Hovekamp, T.B., 2002: Experimental and numerical investigation of porous media flow with regard to the emulsion process. PhD thesis, 1-101.

Hsu, S.J.C., Aspects of piping resistance to seepage in clayey soils. International Conference on Soil Mechanics and Foundation Engineering, Stockholm, vol 10 (1), p-421-428, 1981.

Indraratna, B, Raut, AK \& Khabbaz, H, Constriction-Based Retention Criterion for Granular Filter Design, Journal of Geotechnical and Geoenvironmental Engineering, 2007, 133(3), 266 276

Kanarska, Y., I. Lomov, L. Glascoe, J. Morris, T. Antoun, R. Hall, S. Woodson, J. Fortune, M. E. Hynes, Performance of Damaged Soil-Concrete Wraparound Dam Sections under Dynamic Loading, US Society on Dams (USSD) Annual Meeting and Conference Sacramento, CA, United States April 12, 2010 through April 16, 2010.

Kanarska Y., I. Lomov, T. Antoun. Mesoscale simulations of particulate flows with parallel distributed Lagrange multiplier technique, Computers and Fluids, 48(1), 16-29, 2011 
Kang Q. J., D. Zhang, and S. Chen. "Simulation of dissolution and precipitation in porous media." Journal of Geophysical Research, 108, 1-10 (2003)

Kleinstreuer C., Paller M., Ponzio R. J. (1983) Computer simulation of transport phenomena during dialysis deglycerolization of red blood cells. Computers and Biomedical Research, 1983, 16(1), 29-39.

Lamb, S.H., 1945: Hydrodynamics, 6th ed., Dover Edition, 755p.

Li J., S.S. Pandiella, G. M. Campbell, C. Webb., 2003: Separation of particulate solids by screening -- a discrete particle simulation. Numerical Modeling in Micromechanics via Particle Methods. H. Konietzky, Editor, 93-100.

Lobkovsky A., A. Orpe, R. Molloy, A. Kudrolli, D. Rothman. "Erosion of a granular bed driven by laminar fluid flow." Journal of Fluid Mechanics, 605, 47-58, 2008.

Martin, J.J., W.L. Mccabe, C.C. Monrad, 1951: Pressure drop through stacked spheres: Effect of orientation. Chemical Engineering Progress, 47(2): 91-94. Engineering with Computers, 22(3-4), 181-195.

Meyer-Peter, E and R. Müller. "Formulas for bed-load transport." Proceedings of the 2nd Meeting of the International Association for Hydraulic Structures Research, 39-64, 1948.

Miedema S.A. An analytical method to determine scour. WEDA XXVIII\&Texas A\&M 39, 2008.

Ouriemi M, Aussillous P., Guazzelli E. "Sediment dynamics. Part 1. Bed-load transport by laminar shearing flows". Fluid Mechanics, 636, 295-319, 2009

Patankar N. A. "A formulation for fast computations of rigid particulate flows." Center of Turbulent. Research, Annual Research Briefs, 185-196, 2001

Peskin C.S., 1977: Numerical analysis of blood flow in the heart. Journal of computational physics, 25, p-220-252.

Peskin, C.S. The immersed boundary method. Acta Num., 11, 479, 2002.

Plessis J., 2001: Analytical Quantification of Coefficients in the Ergun Equation for Fluid Friction in a Packed Bed. Transp. Porous Media, 16, 189-207.

Potter M. and D. C. Wiggert. Fluid Mechanics, 248, 2008

Regazzoni P.-L. (2008) Report on the work concerning the tests of soils with the JET and the HET realized on E.R.D.C. and comparison with the tests realized on USBR and ARS. p 30. 
Rajagopalan R., Tien, C., Trajectory analysis of deep-bed filtration with the sphere in a cell porous media model, AIChE J., 22(3), 523-533, 1976

Rodda, K.V., Harlan, R.D., Pardini, R.J., Performance of Austrian Dam during October 17, 1989 Loma Prieta Earthquake. USCOLD newsletter, March, US Committee on Large Dams, Denver, Colorado, 1990

Saxena K.R. and Sharma V.M., DAMS: Incidents and Accidents, A.A. Balkema Publishers, 2005.

Sharma N., Patankar N.A. (2005) A fast computation technique for the direct numerical simulation of rigid particle flows. J. Comp. Phys., 2005, 205, 439-457.

Sherard J.L., L. P. Dunnigan, J. R. Talbot "Basic properties of sand and gravel filters" Journal of Geotechnical Engineering, 110 (6), 684-700, 1984

Sherard J.L., L. P. Dunnigan, J. R. Talbot "Filters for silts and clays" Journal of Geotechnical Engineering, 110 (6), 701-718, 1984

Shields, A. Anwendung der Aehnlichkeitsmechanik und der Turbulenzforschung auf die Geschiebebewegung [Application of similarity principles and turbulence research to bed-load movement]. Mitteilungen der Preußischen Versuchsanstalt für Wasserbau. 26. Berlin: Preußischen Versuchsanstalt für Wasserbau, 1936

Solava S. and Delatte N. (2003) Lessons from the Failure of the Teton Dam, Proceedings of the 3rd ASCE Forensics Congress, October 19 - 21, 2003, San Diego, California.

Soulsby R. Dynamics of marine sands. Thomas Telford Publications, London, United Kingdom, 1997

Stokes, G., 1966: On the effect of the internal friction of fluids on the motion of pendulums. Cambridge Philos. Trans, 9, 8-106.

Syamlal M., W. Rogers and T.J.O. Brien, MFIX Documentation: Theory Guide U.S. Department of Energy (DOE), Morgantown Energy Technology Center, Morgantown, West Virginia, 1993.

Tartakovsky A. M., P. Meakin, T. D. Scheibe, and B. D. Wood. “A smoothed particle hydrodynamics model for reactive transport and mineral precipitation in porous and fractured porous media". Water Resources and Research , 43, 1-18, 2007

Tsuji Y., T. Kawaguchi and T. Tanaka, 1993: Discrete particle simulation of two-dimensional fluidized bed. Powder Technology, 77, 79-87. 
Tuley R., M. Danby, J. Shrimpton, M. Palmer 2010: On the optimal numerical time integration for Lagrangian DEM within implicit flow solvers. Computers and Chemical Engineering, v 34, p 886-899, 2010

USACE Engineer Manual 1110-2-1901, Engineering and Design. Seepage analysis and control for dams. 392 pages.

USCOLD, Austrian Dam, California, USA. Observed performance of dams during earthquakes, Denver, Colorado, 1992

Vorwerk J., P.O. Brunn, 1994: Shearing effects for the flow of surfactant and polymer solutions through a packed-bed of spheres. Journal of Non-Newtonian Fluid Mechanics, v51(1), 79-95.

Wachs, A, 2010: A DEM-DLM/FD method for direct numerical simulation of particulate flows: Sedimentation of polygonal isometric particles in a Newtonian fluid with collisions. Computers and Fluids 38(8), 1608-1628.

Wan C.F. and R. Fell. Investigation of rate of erosion of soils in embankment dams. Journal of Geotechnical and Geoenvironmental Engineering, 130(5), 373-380, 2004

Wan, C. F., and Fell, R. (2002) Investigation of rate of erosion of soils in embankment dams. J. of Geotech. and Geoenv. Eng., ACSE, 2002, 130 (4), 373-380.

Whal T. L., Ragazzoni P.L., Erdoan Z. (2008). Determing erosion indices of cohesive soils with the hole erosion test and jet erosion test. USBR Report DSO-08-05.

Wen, C. Y. , Y. H. Yu, A generalized method for predicting the minimum fluidization velocity, AIChE Journal, Volume 12, Issue 3, pages 610-612, May, 1966

White J. A., Borja R. I. (2008) Stabilized low-order finite elements for coupled soliddeformation/fluid-diffusion and their application to fault zone transients. Comput. Methods Appl. Mech. Engrg., v 197, 4353-4366.

Zhao Z., Fernando H.J.S. (2007) Numerical simulation of scour around pipelines using an EulerEuler coupled two phase model. Env. Fluid Dyn., 2007, 7 (2), 121-142.

Zhu H. P., Z. Y. Zhou, R. Y. Yang, A. B. Yu, Discrete particle simulation of particulate systems: Theoretical developments, Chemical Eng. Science, 62, 3378-3396, 2007. 